\title{
Discrete Complex Analysis on Planar Quad-Graphs
}

\author{
Alexander I. Bobenko and Felix Günther
}

\begin{abstract}
We develop further a linear theory of discrete complex analysis on general quad-graphs, extending previous work of Duffin, Mercat, Kenyon, Chelkak and Smirnov on discrete complex analysis on rhombic quad-graphs. Our approach based on the medial graph leads to generalizations as well as to new proofs of previously known discrete analogs of classical theorems. New results include in particular discretizations of Green's first identity and Cauchy's integral formula for the derivative of a holomorphic function. Another contribution is a discussion on the product of discrete holomorphic functions that is itself discrete holomorphic in a specific sense. In this paper, we focus on planar quad-graphs, but many notions and theorems can be easily adapted to discrete Riemann surfaces. In the case of planar parallelogram-graphs with bounded interior angles and bounded ratio of side lengths explicit formulae for a discrete Green's function and discrete Cauchy's kernels are obtained. This slightly generalizes the previous results on rhombic lattices. When we further restrict to the integer lattice of a two-dimensional skew coordinate system a discrete Cauchy's integral formulae for higher order derivatives is derived.
\end{abstract}

Keywords Discrete complex analysis - Quad-graph • Green's function · Cauchy's integral formulae $\cdot$ Parallelogram-graph

\section{Introduction}

Linear theories of discrete complex analysis look back on a long and varied history. We refer here to the survey of Smirnov [24]. Already Kirchhoff's circuit laws describe a discrete harmonicity condition for the potential function whose gradient describes the current flowing through the electric network. A notable application

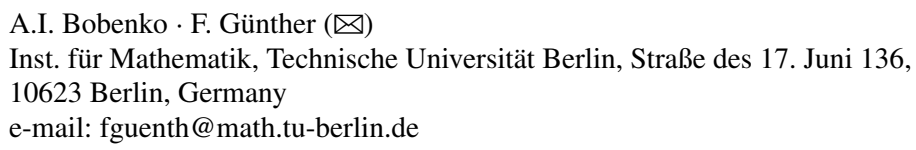

A.I. Bobenko

e-mail: bobenko@math.tu-berlin.de 
of Kirchhoff's laws in geometry was the article [4] of Brooks, Smith, Stone, and Tutte, who used coupled discrete harmonic functions (in fact, discrete holomorphic functions) to construct tilings of rectangles into squares with different integral side lengths. Discrete harmonic functions on the square lattice were studied by a number of authors in the 1920s, including Courant, Friedrichs, and Lewy, who showed convergence of solutions of the Dirichlet boundary value problem to their corresponding continuous counterpart [8].

Discrete holomorphic functions on the square lattice were studied by Isaacs [14]. He proposed two different definitions for holomorphicity. The first one is not symmetric on the square lattice, but it becomes symmetric on the triangular lattice obtained by inserting all southwest-to-northeast diagonals. Dynnikov and Novikov studied an equivalent notion in [11]. His second definition was reintroduced by Lelong-Ferrand in [12]. She developed the theory to a level that allowed her to prove the Riemann mapping theorem using discrete methods [18]. Duffin also studied discrete complex analysis on the square grid [9] and was the first who extended the theory to rhombic lattices [10]. Mercat [19], Kenyon [16], Chelkak and Smirnov [6] resumed the investigation of discrete complex analysis on rhombic lattices or, equivalently, isoradial graphs. In these settings, it was natural to split the real and the imaginary part of a discrete holomorphic function to the two vertex sets of a bipartite decomposition.

Some two-dimensional discrete models in statistical physics exhibit conformally invariant properties in the thermodynamical limit. Such conformally invariant properties were established by Smirnov for site percolation on a triangular grid [25] and for the random cluster model [26], by Chelkak and Smirnov for the Ising model [7], and by Kenyon for the dimer model on a square grid (domino tiling) [15]. In all cases, linear theories of discrete analytic functions on regular grids were highly important. Kenyon [16] as well as Chelkak and Smirnov [6] obtained important analytic results that were instrumental in the proof that the critical Ising model is universal, i.e., that the scaling limit is independent of the shape of the lattice [7]. Already Mercat related the theory of discrete complex analysis to the Ising model and investigated criticality [19].

Important non-linear discrete theories of complex analysis involve circle packings or, more generally, circle patterns. Rodin and Sullivan proved that the Riemann mapping of a complex domain to the unit disk can be approximated by circle packings [22]. A similar result for isoradial circle patterns, even with irregular combinatorics, is due to Bücking [5]. In [2] it was shown that discrete holomorphic functions describe infinitesimal deformations of circle patterns. Moreover, in the case of parallelogramgraphs it was discussed that the corresponding theory is integrable by embedding the quad-graph into $\mathbb{Z}^{n}$.

Our setup in Sect. 2 is a strongly regular cellular decomposition of the complex plane into rectilinear quadrilaterals, called quad-graph. The medial graph of a quad-graph plays a crucial role in our work. It provides the connection between the notions of discrete derivatives of Kenyon [16], Mercat [20], Chelkak and Smirnov [6], extended from rhombic to general quad-graphs, and discrete differential forms and discrete exterior calculus as suggested by Mercat $[19,21]$. Our treatment of discrete differential forms is close to what Mercat proposed in [21]. However, our version 
of discrete exterior calculus is slightly more general. Having introduced the basic notations in the first two sections and the discrete exterior derivative in Sect.2.3.1, we come to Proposition 2.13. There, it is described how the medial graph allows to multiply discrete holomorphic functions to a function that is discrete holomorphic in a certain sense, in particular that it fulfills (discrete) Morera's theorem.

The medial graph approach turns out to be quite useful for integration theory. Theorem 2.16 shows that the discrete exterior derivative is a derivation of the discrete wedge product. Many further results rely on this result and discrete Stokes' Theorem 2.9. In particular, this concerns discrete Green's identities (Theorem 2.23). A discretization of Green's second identity was one ingredient in the proof of Skopenkov's convergence result in [23]. Before the theorem is proved, we introduce the discrete wedge product, the discrete Hodge star, and the discrete Laplacian in Sects. 2.3 and 2.4 .

Skopenkov's results on the existence and uniqueness of solutions to the discrete Dirichlet boundary value problem [23] help us to prove Theorem 2.30. This theorem states surjectivity of the discrete derivatives and the discrete Laplacian seen as linear operators. This implies in particular the existence of discrete Green's functions and discrete Cauchy's kernels. Furthermore, discrete Cauchy's integral formulae for discrete holomorphic functions are derived in Theorem 2.35 and for the discrete derivative of a discrete holomorphic function on the vertices of the quad-graph in Theorem 2.36. Note that discrete Cauchy's integral formula was used by Chelkak and Smirnov to derive certain asymptotic estimates in [7].

Section 3 is devoted to discrete complex analysis on planar parallelogram-graphs. There, explicit formulae for discrete Green's functions and discrete Cauchy's kernels with asymptotics similar to the functions in the rhombic case $[5,6,16]$ are given (Theorems 3.7, 3.8, and 3.10). The general assumption is that the interior angles and the ratio of side lengths of all parallelograms are bounded. The construction of these functions is based on the discrete exponential introduced by Kenyon on quasicrystallic rhombic quad-graphs [16] and its extension to quasicrystallic parallelogramgraphs [2].

In the end, we close with the very special case of the integer lattice of a skew coordinate system in the complex plane. In this case, discrete Cauchy's integral formulae for higher order discrete derivatives of a discrete holomorphic function are derived in Theorem 3.11.

\section{Discrete Complex Analysis on Planar Quad-Graphs}

Although we focus on planar quad-graphs in this paper, many of our notions and theorems generalize to discrete Riemann surfaces. A corresponding linear theory of discrete Riemann surfaces is discussed in the subsequent paper [1] and can be found in more detail in the thesis [13]. 


\subsection{Basic Definitions and Notation}

The aim of this section is to introduce first planar quad-graphs and some basic notation in Sect. 2.1.1 and then to discuss the medial graph in Sect.2.1.2.

\subsubsection{Planar Quad-Graphs}

Definition A planar quad-graph $\Lambda$ without boundary is an infinite graph embedded into the complex plane $\mathbb{C}$ such that all edges are straight line segments and such that all faces are quadrilaterals which may be non-convex. In addition, we assume that $\Lambda$ induces a cellular decomposition of the whole complex plane that is locally finite, i.e., a compact subset of $\mathbb{C}$ contains only finitely many quadrilaterals, and strongly regular, i.e., two different faces are either disjoint or share exactly one vertex or share exactly one edge (but not two edges).

Let $V(\Lambda)$ denote the set of vertices, $E(\Lambda)$ the set of edges, and $F(\Lambda)$ the set of faces of $\Lambda$.

It is well known that any planar quad-graph is bipartite. We fix one decomposition of the vertices of $\Lambda$ into two independent sets and refer to the vertices of this decomposition as black and white vertices, respectively.

Definition Let $\Gamma$ and $\Gamma^{*}$ be the graphs defined on the black and white vertices where $v v^{\prime}$ is an edge of $\Gamma$ (or $\Gamma^{*}$ ) if and only if its two black (or white) endpoints are vertices of a single face.

Remark The assumption of strong regularity guarantees that any edge of $\Gamma$ or $\Gamma^{*}$ is the (possibly outer) diagonal of exactly one quadrilateral.

In order to make the duality between $\Gamma$ and $\Gamma^{*}$ apparent, we consider just for this paragraph the edges of $\Gamma$ or $\Gamma^{*}$ as curves lying totally inside the face they are a diagonal of. Then, any black edge of $\Gamma$ corresponds to the white edge of $\Gamma^{*}$ that crosses it and vice versa. The black and white vertices are in one-to-one correspondence to the faces of $\Gamma^{*}$ and $\Gamma$ they are contained in.

Definition The complex number assigned to a vertex of $\Lambda$ is the corresponding complex value of its embedding in $\mathbb{C}$. To oriented edges of $\Lambda, \Gamma, \Gamma^{*}$ we assign the complex numbers determined by the difference of the complex numbers assigned to their two endpoints.

Remark For simplicity, we perform our calculations hereafter directly with the vertices and oriented edges of $\Lambda, \Gamma, \Gamma^{*}$ rather than replacing them with their corresponding complex values.

Definition $\diamond:=\Lambda^{*}$ is the dual graph of $\Lambda$. 
In general, we do not specify a planar embedding of the dual graph $\diamond$. We will just identify vertices or faces of $\diamond$ with their corresponding dual faces and vertices of $\Lambda$, respectively. However, in the particular case that all quadrilaterals are parallelograms, it makes sense to identify the vertices of $\diamond$ (i.e., faces of the quad-graph $\Lambda$ ) with the centers of the parallelograms. Here, the center of a parallelogram is the point of intersection of its two diagonals. Further details will be given in Sects. 2.2.1 and 2.2.3.

Definition If a vertex $v \in V(\Lambda)$ is a vertex of a quadrilateral $Q \in F(\Lambda)$, we write $Q \sim v$ or $v \sim Q$ and say that $v$ and $Q$ are incident to each other.

Throughout our paper, we will denote the vertices of a single quadrilateral $Q$ by $b_{-}, w_{-}, b_{+}, w_{+}$in counterclockwise order, where $b_{ \pm} \in V(\Gamma)$ and $w_{ \pm} \in V\left(\Gamma^{*}\right)$.

Definition For a quadrilateral $Q \in F(\Lambda)$ we define

$$
\rho_{Q}:=-i \frac{w_{+}-w_{-}}{b_{+}-b_{-}} .
$$

Moreover, let

$$
\varphi_{Q}:=\arccos \left(\operatorname{Re}\left(i \frac{\rho_{Q}}{\left|\rho_{Q}\right|}\right)\right)=\arccos \left(\operatorname{Re}\left(\overline{\frac{\left(b_{+}-b_{-}\right)}{\left.\left|b_{+}-b_{-}-\right| w_{+}-w_{-}\right)}}\right)\right)
$$

be the angle under which the diagonal lines of $Q$ intersect.

Note that $0<\varphi_{Q}<\pi$. Figure 1 shows a finite bipartite quad-graph together with the notations we have introduced for a single quadrilateral $Q$ and the notations we are using later for the star of a vertex $v$, i.e., the set of all faces incident to $v$.

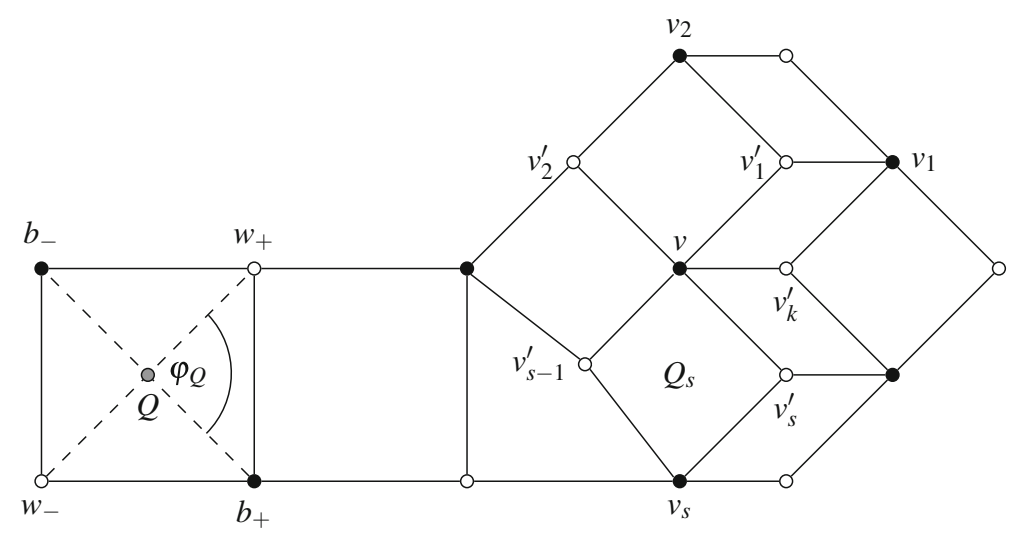

Fig. 1 Bipartite quad-graph with notations 
In addition, we denote by $\nabla_{0}$ a subgraph of $\diamond$ that we always assume to be connected and by $V\left(\nabla_{0}\right) \subseteq V(\diamond)$ the corresponding subset of faces of the quadgraph $\Lambda$. Through our identification $V(\diamond) \cong F(\Lambda)$, we can call the elements of $V(\diamond)$ quadrilaterals and identify them with the corresponding faces of $\Lambda$.

Definition $\nabla_{0} \subseteq \diamond$ is said to form a simply-connected closed region if the union of all quadrilaterals in $V\left(\diamond_{0}\right)$ is a simply-connected closed region in $\mathbb{C}$.

Definition Let $\Lambda_{0}$ be the subgraph of $\Lambda$ whose vertices and edges are exactly the corners and edges of the quadrilaterals in $V\left(\nabla_{0}\right)$. The interior faces of $\Lambda_{0}$ are given by $V\left(\diamond_{0}\right)$. Let $\Gamma_{0}$ and $\Gamma_{0}^{*}$ denote the subgraphs of $\Gamma$ and of $\Gamma^{*}$ whose edges are the diagonals of quadrilaterals in $V\left(\diamond_{0}\right)$ and who do not contain isolated vertices.

Remark Since $\nabla_{0} \subseteq \diamond$ is connected, $\Gamma_{0}$ and $\Gamma_{0}^{*}$ are connected as well. Indeed, if $Q, Q^{\prime} \in V\left(\nabla_{0}\right)$ are two quadrilaterals adjacent in $\nabla_{0}$, then the corresponding subgraphs on $\Gamma_{0}$ and $\Gamma_{0}^{*}$ consisting of the three black and white vertices of $Q$ and $Q^{\prime}$ are paths of two edges each.

Definition $\Lambda_{0}$ is called a (planar) quad-graph induced by the subgraph $\nabla_{0} \subseteq \vartheta_{\text {. Its }}$ boundary $\partial \Lambda_{0}$ is the subgraph of $\Lambda_{0}$ that consists of all the edges of $\Lambda_{0}$ that belong to both a quadrilateral in $V\left(\diamond_{0}\right)$ and one in $V(\diamond) \backslash V\left(\diamond_{0}\right)$.

Remark If $\nabla_{0} \subseteq \diamond$ is finite and forms a simply-connected closed region, then the set of all interior faces of $\Lambda_{0}$ is homeomorphic to a disk and $\partial \Lambda_{0}$ is a closed broken line without self-intersections.

\subsubsection{Medial Graph}

Definition The medial graph $X$ of $\Lambda$ is defined as follows. Its vertex set is given by all the midpoints of the edges of $\Lambda$ embedded in $\mathbb{C}$, and two vertices $x, x^{\prime}$ are adjacent if and only if the corresponding edges belong to the same face $Q$ of $\Lambda$ and have a vertex $v \in V(\Lambda)$ in common. We denote this edge by $[Q, v]$. Taking $[Q, v]$ as a straight line segment if $v$ is a convex corner of the quadrilateral $Q$ and as a curve lying inside $Q$ that does not intersect the three other edges $\left[Q, v^{\prime}\right]\left(v^{\prime} \sim Q, v^{\prime} \neq v\right)$ inside $Q$ if $v$ is a concave corner, we get an embedding of $X$ into $\mathbb{C}$. Then, the set $F(X)$ of faces of $X$ is in bijection with $V(\Lambda) \cup V(\diamond)$ : A face $F_{v}$ of $X$ corresponding to $v \in V(\Lambda)$ has the midpoints of edges of $\Lambda$ incident to $v$ as vertices, and a face $F_{Q}$ of $X$ corresponding to $Q \in F(\Lambda) \cong V(\diamond)$ has the midpoints of the four edges of $\Lambda$ belonging to $Q$ as vertices. The vertices of $F_{Q}$ and $F_{v}$ are colored gray in Fig. 2.

Remark Clearly, any pair $Q \sim v$ of a face and an incident vertex of $\Lambda$ corresponds to an edge $[Q, v]$ of $X$. Moreover, a face $F_{Q}$ lies inside $Q$ and $v$ is contained in $F_{v}$.

Definition As for the vertices and edges of $\Lambda$, we assign to a vertex of $X$ the complex number corresponding to its position in $\mathbb{C}$, and to an oriented edge of $X$ we assign the difference of the two endpoints. 


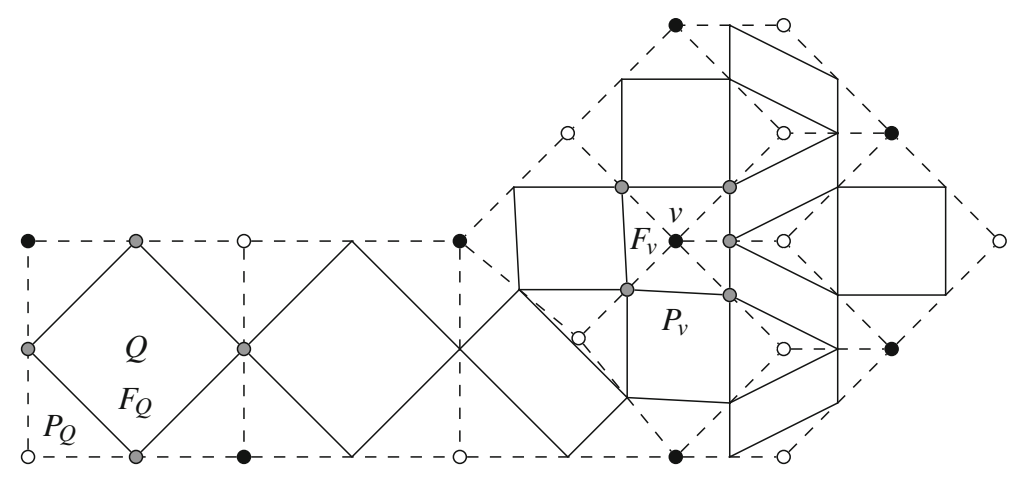

Fig. 2 Bipartite quad-graph (dashed) with medial graph (solid)

Even though not all edges of $X$ might be straight line segments, we actually think of them as being straight since we assign the vector of its endpoints to it if the edge is oriented. In this sense, any face $F_{Q}, Q \in F(\Lambda)$, is a parallelogram due to Varignon's theorem. Moreover, the complex number assigned to the edge $\left[Q, v_{0}\right], v_{0} \sim Q$, if oriented from the midpoint of the edge $v_{0} v_{-}^{\prime}$ to the one of the edge $v_{0} v_{+}^{\prime}$ of $\Lambda$ is just half of $e=v_{+}^{\prime}-v_{-}^{\prime}$. We will say that $\left[Q, v_{0}\right]$ is parallel to $e$ (disregarding the orientation), as it would be if we considered all edges of $X$ as straight line segments.

Remark If all quadrilaterals of $\Lambda$ are convex, then the embedding of $X$ given above consists of straight line segments only. If no Varignon parallelogram of a non-convex quadrilateral contains another vertex of $X$ apart from its corners, then the corresponding straight line realization gives an embedding equivalent to the one above. In this case, the face $F_{v}$ of $X$ corresponding to a vertex $v \in V(\Lambda)$ that is a concave corner of a quadrilateral does not contain $v$ any longer. However, if such a Varignon parallelogram contains an additional vertex of $X$, then connecting adjacent vertices of $X$ by straight line segments does not yield an embedding of $X$.

Definition For a connected subgraph $\nabla_{0} \subseteq \vartheta$, we denote by $X_{0} \subseteq X$ the connected subgraph of $X$ consisting of all edges $[Q, v]$ where $Q \in V\left(\nabla_{0}\right)$ and $v$ is a corner of $Q$. The boundary $\partial X_{0}$ is the subgraph of $X_{0}$ whose edges are exactly those $[Q, v]$ where $v \in V\left(\partial \Lambda_{0}\right)$ and $Q \in V\left(\nabla_{0}\right)$ is incident to $v$. We consider the orientation on the boundary $\partial X_{0}$ that is induced by orienting any of its edges $[Q, v]$ counterclockwise with regard to $Q$. For a finite collection $F$ of faces of $X_{0}, \partial F$ denotes the union of all counterclockwise oriented boundaries of faces in $F$, where oriented edges in opposite directions cancel each other out.

Remark In the case that all quadrilaterals in $V\left(\diamond_{0}\right)$ are convex, $X_{0}$ consists of all straight edges of $X$ that lie inside the closed region formed by the quadrilaterals in $V\left(\triangleright_{0}\right)$. In any case, the medial graph $X$ corresponds to a (strongly regular and locally finite) cellular decomposition of the plane in a canonical way. In particular, we can talk about a topological disk $D$ in $F\left(X_{0}\right)$ as a finite collection of faces of $X_{0}$ that form 
a closed region homeomorphic to a disk, and we can consider its counterclockwise oriented boundary $\partial D$ as a subgraph of $X_{0}$.

Definition For $v \in V(\Lambda)$ and $Q \in F(\Lambda)$, let $P_{v}$ and $P_{Q}$ be the closed paths on $X$ connecting the midpoints of edges of $\Lambda$ incident to $v$ and $Q$, respectively, in counterclockwise direction. In Fig. 2, their vertices are colored gray. We say that $P_{v}$ and $P_{Q}$ are discrete elementary cycles.

\subsection{Discrete Holomorphicity}

To motivate the definition of discrete holomorphicity due to Mercat [21] that was also used previously in the rhombic setting by Duffin [10] and others, let us have a short look to the classical theory. There, a real differentiable complex function $f$ defined on an open subset of the complex plane is holomorphic if and only if in any point all directional derivatives coincide. Moreover, holomorphic functions with nowhere-vanishing derivative preserve angles, and at a single point, infinitesimal lengths are uniformly scaled.

Definition Let $Q \in V(\diamond) \cong F(\Lambda)$ and $f$ be a complex function on the vertices $b_{-}, w_{-}, b_{+}, w_{+}$of the quadrilateral $Q . f$ is said to be discrete holomorphic at $Q$ if the discrete Cauchy-Riemann equation is satisfied:

$$
\frac{f\left(b_{+}\right)-f\left(b_{-}\right)}{b_{+}-b_{-}}=\frac{f\left(w_{+}\right)-f\left(w_{-}\right)}{w_{+}-w_{-}} .
$$

Definition Let $f: V\left(\Lambda_{0}\right) \rightarrow \mathbb{C}$. $f$ is said to be discrete holomorphic if $f$ is discrete holomorphic at all $Q \in V\left(\diamond_{0}\right)$.

Note that if a discrete holomorphic function $f$ does not have the same value on both black vertices $b_{-}$and $b_{+}$, then it preserves the angle $\varphi_{Q}$ and $f$ uniformly scales the lengths of the diagonals of $Q$. However, the image of $Q$ under $f$ might be a degenerate or self-intersecting quadrilateral.

We immediately see that for discrete holomorphicity, only the differences at black and at white vertices matter. Hence, we should not consider constants on $V(\Lambda)$, but biconstants [20] determined by each a value on $V(\Gamma)$ and $V\left(\Gamma^{*}\right)$.

Definition A function $f: V\left(\Lambda_{0}\right) \rightarrow \mathbb{C}$ that is constant on $V\left(\Gamma_{0}\right)$ and constant on $V\left(\Gamma_{0}^{*}\right)$ is said to be biconstant.

In the following, we will define discrete analogs of $\partial, \bar{\partial}$, first of functions on $V(\Lambda)$ in Sect.2.2.1 and later of functions on $V(\diamond)$ in Sect.2.2.3. Before, we introduce discrete differential forms in Sect. 2.2.2. 


\subsubsection{Discrete Derivatives of Functions on the Vertices of $\boldsymbol{A}$}

Definition Let $Q \in V(\diamond) \cong F(\Lambda)$, and let $f$ be a complex function on its vertices $b_{-}, w_{-}, b_{+}, w_{+}$. The discrete derivatives $\partial_{\Lambda} f, \bar{\partial}_{\Lambda} f$ are defined by

$$
\begin{aligned}
& \partial_{\Lambda} f(Q):=\lambda_{Q} \frac{f\left(b_{+}\right)-f\left(b_{-}\right)}{b_{+}-b_{-}}+\bar{\lambda}_{Q} \frac{f\left(w_{+}\right)-f\left(w_{-}\right)}{w_{+}-w_{-}}, \\
& \bar{\partial}_{\Lambda} f(Q):=\bar{\lambda}_{Q} \frac{f\left(b_{+}\right)-f\left(b_{-}\right)}{\overline{b_{+}-b_{-}}}+\lambda_{Q} \frac{f\left(w_{+}\right)-f\left(w_{-}\right)}{\overline{w_{+}-w_{-}}},
\end{aligned}
$$

where $2 \lambda_{Q}:=\exp \left(-i\left(\varphi_{Q}-\frac{\pi}{2}\right)\right) / \sin \left(\varphi_{Q}\right)$.

Remark Clearly, biconstant functions have vanishing discrete derivatives.

If the quadrilateral $Q$ is a rhombus, then $\varphi_{Q}=\pi / 2$ and $\lambda_{Q}=1 / 2$. Therefore, the definition above then reduces to the previous one given by Chelkak and Smirnov [6]. The definition of discrete derivatives matches the notion of discrete holomorphicity; and the discrete derivatives approximate their smooth counterparts correctly up to order one for general quad-graphs and up to order two for parallelogram-graphs:

Proposition 2.1 Let $Q$ be a face of the quad-graph $\Lambda$ and $f$ be a complex function on its vertices $b_{-}, w_{-}, b_{+}, w_{+}$.

(i) $f$ is discrete holomorphic at $Q$ if and only if $\bar{\partial}_{\Lambda} f(Q)=0$.

(ii) For the function $f(v)=v, \bar{\partial}_{\Lambda} f(Q)=0$ and $\partial_{\Lambda} f(Q)=1$.

(iii) If $Q$ is a parallelogram and $f(v)=v^{2}$, then $\bar{\partial}_{\Lambda} f(Q)=0, \partial_{\Lambda} f(Q)=2 \hat{Q}$.

(iv) If $Q$ is a parallelogram and $f(v)=|v|^{2}$, then $\bar{\partial}_{\Lambda} f(Q)=\overline{\partial_{\Lambda} f(Q)}=\hat{Q}$.

In parts (iii) and (iv), $\hat{Q}$ denotes the center of the parallelogram $Q$.

Proof (i) We observe that

$$
\begin{aligned}
& \frac{2 \sin \left(\varphi_{Q}\right) \bar{\lambda}_{Q}}{\overline{b_{+}-b_{-}}}=\frac{\exp \left(i\left(\varphi_{Q}-\frac{\pi}{2}\right)\right)}{\overline{b_{+}-b_{-}}}=\frac{-i \exp \left(i \varphi_{Q}\right) \frac{b_{+}-b_{-}}{\left|b_{+}-b_{-}\right|}}{\left|b_{+}-b_{-}\right|}=\frac{-i\left(w_{+}-w_{-}\right)}{\left|w_{+}-w_{-}\right|\left|b_{+}-b_{-}\right|}, \\
& \frac{2 \sin \left(\varphi_{Q}\right) \lambda_{Q}}{\overline{w_{+}-w_{-}}}=\frac{\exp \left(i\left(\frac{\pi}{2}-\varphi_{Q}\right)\right)}{\overline{w_{+}-w_{-}}}=\frac{i \exp \left(-i \varphi_{Q}\right) \frac{w_{+}-w_{-}}{\left|w_{+}-w_{-}\right|}}{\left|w_{+}-w_{-}\right|}=\frac{i\left(b_{+}-b_{-}\right)}{\left|w_{+}-w_{-}\right|\left|b_{+}-b_{-}\right|} .
\end{aligned}
$$

So if we multiply $\bar{\partial}_{\Lambda} f(Q)$ by $2 i\left|w_{+}-w_{-}\right|\left|b_{+}-b_{-}\right| \sin \left(\varphi_{Q}\right) \neq 0$, we obtain

$$
\left(w_{+}-w_{-}\right)\left(f\left(b_{+}\right)-f\left(b_{-}\right)\right)-\left(b_{+}-b_{-}\right)\left(f\left(w_{+}\right)-f\left(w_{-}\right)\right),
$$

which vanishes if and only if the discrete Cauchy-Riemann equation is satisfied.

(ii) Clearly, $f(v)=v$ satisfies the discrete Cauchy-Riemann equation. By the first part, $\bar{\partial}_{\Lambda} f(Q)=0$. Due to $2 \sin \left(\varphi_{Q}\right)=\exp \left(-i\left(\varphi_{Q}-\frac{\pi}{2}\right)\right)+\exp \left(i\left(\varphi_{Q}-\frac{\pi}{2}\right)\right)$, $\partial_{\Lambda} f(Q)$ simplifies to $\lambda_{Q}+\bar{\lambda}_{Q}=1$. 
(iii) For the function $f(v)=v^{2}$, the discrete Cauchy-Riemann equation is equivalent to $b_{+}+b_{-}=w_{+}+w_{-}$. But since $Q$ is a parallelogram, both $\left(b_{+}+b_{-}\right) / 2$ and $\left(w_{+}+w_{-}\right) / 2$ equal its center $\hat{Q}$. Thus, $f$ is discrete holomorphic at $Q$ and

$$
\partial_{\Lambda} f(Q)=\lambda_{Q}\left(b_{+}+b_{-}\right)+\bar{\lambda}_{Q}\left(w_{+}+w_{-}\right)=2 \hat{Q}\left(\lambda_{Q}+\bar{\lambda}_{Q}\right)=2 \hat{Q} .
$$

(iv) Since $f$ is a real function, $\bar{\partial}_{\Lambda} f(Q)=\overline{\partial_{\Lambda} f(Q)}$ follows straight from the definition. Let $z \in \mathbb{C}$ be arbitrary. If $g(v):=v \bar{z}$, then $\partial_{\Lambda} g(Q)=\bar{z}$ and $\partial_{\Lambda} \bar{g}(Q)=0$ by the second part. So if we define the function $h(v):=|v-z|^{2}=|v|^{2}-v \bar{z}-\bar{v} z+|z|^{2}$, then $\overline{\partial_{\Lambda} h(Q)}=\overline{\partial_{\Lambda} f(Q)}-z$ using the second part and observing that constant functions have vanishing derivatives. Hence, the statement is invariant under translation, and it suffices to consider the case $\hat{Q}=0$. Then, $b_{+}=-b_{-}$and $w_{+}=-w_{-}$ since $Q$ is a parallelogram. It follows that $f\left(b_{-}\right)=f\left(b_{+}\right)$and $f\left(w_{-}\right)=f\left(w_{+}\right)$, so $\partial_{\Lambda} f(Q)=0$.

Our first discrete analogs of classical theorems are immediate consequences of the discrete Cauchy-Riemann equation:

Proposition 2.2 Let $f: V\left(\Lambda_{0}\right) \rightarrow \mathbb{C}$ be discrete holomorphic.

(i) If $f$ is purely imaginary or purely real, then $f$ is biconstant.

(ii) If $\partial_{\Lambda} f \equiv 0$, then $f$ is biconstant.

Proof (i) Let us assume that $f$ is not biconstant. Then, without loss of generality, $f$ is not constant on $\Gamma_{0}$. Since $\Gamma_{0}$ is connected, there are two adjacent vertices $b_{-}, b_{+}$ of $\Gamma_{0}$ such that $f\left(b_{+}\right) \neq f\left(b_{-}\right)$. Let $b_{-}, w_{-}, b_{+}, w_{+} \in V\left(\Lambda_{0}\right)$ be the vertices of the interior face of $\Lambda_{0}$ with black diagonal $b_{-} b_{+}$. Due to the discrete Cauchy-Riemann equation,

$$
\frac{f\left(w_{+}\right)-f\left(w_{-}\right)}{f\left(b_{+}\right)-f\left(b_{-}\right)}=\frac{w_{+}-w_{-}}{b_{+}-b_{-}} .
$$

The left hand side is real and well-defined since $f$ is purely imaginary or purely real and $f\left(b_{+}\right) \neq f\left(b_{-}\right)$. But the right hand side is not, contradicting the assumption that $f$ is not biconstant.

(ii) Since $f$ is discrete holomorphic,

$$
\frac{f\left(b_{+}\right)-f\left(b_{-}\right)}{b_{+}-b_{-}}=\frac{f\left(w_{+}\right)-f\left(w_{-}\right)}{w_{+}-w_{-}} .
$$

$\partial_{\Lambda} f \equiv 0$ then yields that both sides of the discrete Cauchy-Riemann equation equal zero, so $f$ is constant on $V\left(\Gamma_{0}\right)$ and on $V\left(\Gamma_{0}^{*}\right)$ since both graphs are connected.

\subsubsection{Discrete Differential Forms}

In our paper, we mainly consider two type of functions, functions $f: V\left(\Lambda_{0}\right) \rightarrow \mathbb{C}$ and functions $h: V\left(\diamond_{0}\right) \rightarrow \mathbb{C}$. An example for a function on $V\left(\diamond_{0}\right)$ is $\partial_{\Lambda} f$. 
Definition A discrete one-form $\omega$ is a complex function on the oriented edges of the medial graph $X_{0}$ such that $\omega(-e)=\omega(e)$ for any oriented edge $e$ of $X_{0}$. Here, $-e$ denotes the edge $e$ with opposite orientation.

The evaluation of $\omega$ at an oriented edge $e$ of $X_{0}$ is denoted by $\int_{e} \omega$. If $P$ is a directed path in $X_{0}$ consisting of oriented edges $e_{1}, e_{2}, \ldots, e_{n}$, then the discrete integral along $P$ is $\int_{P} \omega=\sum_{k=1}^{n} \int_{e_{k}} \omega$. For closed paths $P$, we write $\oint_{P} \omega$ instead. If $P$ is the oriented boundary of a topological disk $D$ in $F\left(X_{0}\right)$, then we say that the discrete integral is a discrete contour integral with discrete contour $P$.

Since we consider the planar case, one-forms $d z$ and $d \bar{z}$ are globally defined.

Definition The discrete one-forms $d z$ and $d \bar{z}$ are given by $\int_{e} d z=e$ and $\int_{e} d \bar{z}=\bar{e}$ for any oriented edge $e$ of $X$.

It turns out that discrete one-forms that actually come from discrete one-forms on $\Gamma$ and $\Gamma^{*}$ are of particular interest:

Definition A discrete one-form $\omega$ defined on the oriented edges of $X_{0}$ is of type $\diamond$ if for any $Q \in V\left(\nabla_{0}\right)$ there exist complex numbers $p, q$ such that $\omega=p d z+q d \bar{z}$ on all edges $e=[Q, v], v \in V\left(\Lambda_{0}\right)$ incident to $Q . \omega$ is of type $\Lambda$ if for any $v \in V\left(\Lambda_{0}\right)$ there exist complex numbers $p, q$ such that $\omega=p d z+q d \bar{z}$ on all edges $e=[Q, v]$, $Q \in V\left(\diamond_{0}\right)$ incident to $v$.

Remark Discrete one-forms of type $\Lambda$ do not play such an important role as discrete one-forms of type $\diamond$, although they occur as discrete differentials of functions defined on $V\left(\diamond_{0}\right)$. This will become clear in the end of Sect.2.3.2, one of the reasons being that discrete one-forms of type $\Lambda$ are not defined on discrete Riemann surfaces, but discrete one-forms of type $\diamond$ are.

Definition A discrete two-form $\Omega$ is a complex function on the faces of $X_{0}$.

The evaluation of $\Omega$ at a face $F$ of $X_{0}$ is denoted by $\iint_{F} \Omega$. If $S$ is a set of faces $F_{1}, F_{2}, \ldots, F_{n}$ of $X_{0}$, then $\iint_{S} \Omega=\sum_{k=1}^{n} \iint_{F_{k}} \Omega$ is the discrete integral of $\Omega$ over $S$.

As we are mainly interested in functions $f: V\left(\Lambda_{0}\right) \rightarrow \mathbb{C}$ and $h: V\left(\diamond_{0}\right) \rightarrow \mathbb{C}$, discrete two-forms of particular interest are those that vanish on faces of $X_{0}$ corresponding to vertices of either $\diamond$ or $\Lambda$.

Definition A discrete two-form $\Omega$ defined on $F\left(X_{0}\right)$ is of type $\Lambda$ if $\Omega$ vanishes on all faces of $X_{0}$ corresponding to $V\left(\nabla_{0}\right)$ and of type $\diamond$ if $\Omega$ vanishes on all faces of $X$ corresponding to $V\left(\Lambda_{0}\right)$.

Remark These discrete two-forms correspond to functions on $V\left(\Lambda_{0}\right)$ or $V\left(\diamond_{0}\right)$ by the discrete Hodge star that will be defined later in Sect. 2.3.3.

Since we did not give a precise embedding of the medial graph into the complex plane in the general case, we have to specify what the area of a face is. This area includes a factor of two in order to get the same coefficients in the discrete setup as in the smooth case. 
Definition Let $F$ be a face of the medial graph $X$. We define $\operatorname{ar}(F)$ to be twice the Euclidean area of the polygon that results from connecting adjacent vertices of $F$ by straight line segments in the complex plane. In contrast, area $(P)$ will always denote the Euclidean area of a polygon $P$.

Remark As we have mentioned before, our main objects either live on the quad-graph $\Lambda$ or on its dual $\diamond$. Thus, we have to deal with two different cellular decompositions at the same time. The medial graph has the crucial property that its faces are in one-to-one correspondence to vertices of $\Lambda$ and of $\nabla$, i.e., to faces of $\nabla$ and of $\Lambda$. Furthermore, the Euclidean area of the Varignon parallelogram of $Q \in F(\Lambda)$ is just half of the area of $Q$. In some sense, a corresponding statement is true for the cells of $X$ corresponding to vertices of $\Lambda$, i.e., faces of $\diamond$. However, there is not only no canonical embedding of $X$, but also no natural embedding of $\diamond$ in the general setting. But in the particular case of parallelogram-graphs, when we have a canonical embedding of $X$ with rectilinear edges, we can make the statement precise: If an edge $Q Q^{\prime}$ of $\diamond$ is represented by the two line segments that connect the centers of the parallelograms $Q$ and $Q^{\prime}$ with the midpoint of their common edge, then the Euclidean area of the face of $X$ corresponding to a vertex $v \in V(\Lambda) \cong F(\diamond)$ is exactly half of the area of the face of $\diamond$ corresponding to $v$.

In summary, the medial graph allows us to deal with just one decomposition of the complex plane, but we have to count areas twice in order to get the right coefficients as in the continuous setup.

Definition The discrete two-forms $\Omega_{\Lambda}$ and $\Omega_{\diamond}$ are defined as being zero on faces of $X$ corresponding to vertices of $\diamond$ or $\Lambda$, respectively, and defined by

$$
\iint_{F_{v}} \Omega_{\Lambda}=-2 i \operatorname{ar}\left(F_{v}\right) \text { and } \iint_{F_{Q}} \Omega_{\diamond}=-2 i \operatorname{ar}\left(F_{Q}\right)
$$

on faces $F_{v}$ and $F_{Q}$ corresponding to $v \in V(\Lambda)$ or $Q \in V(\diamond)$. As defined above, $\operatorname{ar}(F)$ is twice the Euclidean area of the straight-line embedding of $F_{v}$ or $F_{Q}$.

Remark $\Omega_{\Lambda}$ and $\Omega_{\diamond}$ are the straightforward discretizations of $d z \wedge d \bar{z}$ having in mind that they are essentially defined on faces of $\diamond$ or of $\Lambda$, respectively. It turns out that in local coordinates, we can perform our calculations with $\Omega_{\Lambda}$ and $\Omega_{\diamond}$ in the discrete setting exactly as we do with $d z \wedge d \bar{z}$ in the smooth theory. We will see in Sect. 2.3.2 that $\Omega_{\diamond}$ is indeed the discrete wedge product of $d z$ and $d \bar{z}$ seen as discrete one-forms of type $\nabla$. The same would be true for $\Omega_{\Lambda}$ if we considered $d z$ and $d \bar{z}$ as being of type $\Lambda$, but the discrete wedge product is of interest just for discrete one-forms of type $\diamond$ and we therefore define it just for these forms.

Definition Let $f: V\left(\Lambda_{0}\right) \rightarrow \mathbb{C}, h: V\left(\searrow_{0}\right) \rightarrow \mathbb{C}, \omega$ a discrete one-form defined on the oriented edges of $X_{0}$, and $\Omega_{1}, \Omega_{2}$ discrete two-forms defined on $F\left(X_{0}\right)$ that are of type $\Lambda$ and $\diamond$, respectively. For any oriented edge $e=[Q, v]$ and any faces $F_{v}, F_{Q}$ of $X_{0}$ corresponding to $v \in V\left(\Lambda_{0}\right)$ or $Q \in V\left(\diamond_{0}\right)$, we define the products $f \omega, h \omega$, $f \Omega_{1}$, and $h \Omega_{2}$ by 


$$
\begin{array}{llrl}
\int_{e} f \omega:=f(v) \int_{e} \omega & \text { and } \iint_{F_{v}} f \Omega_{1}:=f(v) \iint_{F_{v}} \Omega_{1}, \iint_{F_{Q}} f \Omega_{1}:=0 \\
\int_{e} h \omega:=h(Q) \int_{e} \omega & \text { and } \iint_{F_{v}} h \Omega_{2}:=0, & \iint_{F_{Q}} h \Omega_{2}:=h(Q) \iint_{F_{Q}} \Omega_{2} .
\end{array}
$$

In the following table, we give a quick overview of various discrete differential forms (most of them will be discussed in Sect.2.3) and state whether they are essentially functions on $V(\Lambda)$ (first column) or functions on $V(\diamond)$ (second column) or entirely objects on the cellular decomposition $X$ (third column). In the first row we find functions, in the second discrete one-forms, and in the third discrete two-forms. So for example, the intersection of the second row with the third column lists discrete one-forms that are entirely objects on $X$ and cannot be reduced to functions on $V(\Lambda)$ or $V(\diamond)$.

\begin{tabular}{l|l|l|l}
\hline \multirow{3}{*}{ functions } & $\Lambda$ & $\diamond$ & $X$ \\
\hline \multirow{4}{*}{ 1-forms } & $f, g: V(\Lambda) \rightarrow \mathbb{C}$ & $h_{1}, h_{2}: V(\diamond) \rightarrow \mathbb{C}$ & $f \cdot g=\int(f d g+g d f)$ \\
\cline { 2 - 4 } & $\partial_{\diamond} h, \partial_{\diamond} h$ & $\partial_{\Lambda} f, \partial_{\Lambda} f$ & \\
\cline { 2 - 4 } & $d h$ & $d f$ & $f d g+g d f$ \\
\cline { 2 - 4 } & $h_{1} d z+h_{2} d \bar{z}$ & $f d z+g d \bar{z}$ & $f h d z$ \\
\cline { 2 - 4 } 2-forms & $\Omega_{\Lambda}$ type $\Lambda$ & $\omega, \omega^{\prime}$ of type $\diamond$ & $f \omega$ \\
\cline { 2 - 4 } & $\star f$ & $\Omega_{\diamond}$ & $d(f h d z)$ \\
\cline { 2 - 4 } & $d \omega$ & $\star h$ & $d(f \omega)$ \\
\cline { 2 - 4 } & $f d \omega$ & $d \eta$ & \\
\hline
\end{tabular}

Remark Although discrete one-forms of type $\Lambda$ or of type $\diamond$ do not live themselves on $\Lambda$ or $\diamond$, they are described by two functions defined on the vertices of $\Lambda$ or $\diamond$, respectively.

\subsubsection{Discrete Derivatives of Functions on the Faces of $\boldsymbol{\Lambda}$}

Before we pass on to discrete derivatives of functions on $V(\diamond)$, we first prove an alternative formula for the discrete derivatives of functions on $V(\Lambda)$.

Lemma 2.3 Let $Q \in V(\diamond) \cong F(\Lambda)$ and $f$ be a complex function on the vertices $b_{-}, w_{-}, b_{+}, w_{+}$of $Q$. Let $P_{Q}$ be the discrete elementary cycle around $Q$ and $F$ the face of $X$ corresponding to $Q$. Then,

$$
\partial_{\Lambda} f(Q)=\frac{-1}{2 i \operatorname{ar}(\mathrm{F})} \oint_{P_{Q}} f d \bar{z} \text { and } \bar{\partial}_{\Lambda} f(Q)=\frac{1}{2 i \operatorname{ar}(\mathrm{F})} \oint_{P_{Q}} f d z .
$$


Proof Since we think of $F$ as a parallelogram (see Sect.2.1.2), its Euclidean area is half of the area of $Q$. So by definition,

$$
\operatorname{ar}(F)=\frac{1}{2}\left|b_{+}-b_{-}\right|\left|w_{+}-w_{-}\right| \sin \left(\varphi_{Q}\right)
$$

Furthermore, $f\left(b_{+}\right)$and $-f\left(b_{-}\right)$are multiplied by the same factor $\overline{\left(w_{+}-w_{-}\right)} / 2$ when evaluating the discrete contour integral $\oint_{P_{Q}} f d \bar{z}$. Therefore, the coefficient in front of $f\left(b_{+}\right)-f\left(b_{-}\right)$in the right hand side of the first equation in the lemma is

$$
i \frac{\overline{w_{+}-w_{-}}}{4 \operatorname{ar}(F)}=-\bar{i} \frac{\overline{w_{+}-w_{-}}}{2 \sin \left(\varphi_{Q}\right)\left|w_{+}-w_{-}\right|\left|b_{+}-b_{-}\right|}=\frac{\exp \left(-i\left(\varphi_{Q}-\frac{\pi}{2}\right)\right)}{2 \sin \left(\varphi_{Q}\right)\left(b_{+}-b_{-}\right)}=\frac{\lambda_{Q}}{b_{+}-b_{-}}
$$

(compare with the proof of Proposition 2.1(i)), which is exactly the coefficient appearing in $\partial_{\Lambda} f(Q)$. Analogously, the coefficients in front of $f\left(w_{+}\right)-f\left(w_{-}\right)$are equal. This shows the first equation. The second one follows from the first, noting that the coefficients in front of $f\left(b_{+}\right)-f\left(b_{-}\right)$and $f\left(w_{+}\right)-f\left(w_{-}\right)$on both sides of the second equation are just complex conjugates of the corresponding coefficients appearing in the first equation.

Inspired by Lemma 2.3 that is illustrated by Fig. 3a, we can now define the discrete derivatives for complex functions on $V(\diamond)$, see Fig. $3 b$.

Definition Let $v \in V(\Lambda)$ and $h$ be a complex function defined on all quadrilaterals that are incident to $v$. Let $P_{v}$ be the discrete elementary cycle around $v$ and $F$ the face of $X$ corresponding to $v$. Then, the discrete derivatives $\partial_{\diamond} h, \bar{\partial}_{\diamond} h$ at $v$ are defined by

$$
\partial_{\diamond} h(v):=\frac{-1}{2 i \operatorname{ar}(F)} \oint_{P_{v}} h d \bar{z} \text { and } \bar{\partial} \diamond h(v):=\frac{1}{2 i \operatorname{ar}(F)} \oint_{P_{v}} h d z .
$$

$h$ is said to be discrete holomorphic at $v$ if $\bar{\partial} \diamond h(v)=0$.

(a)

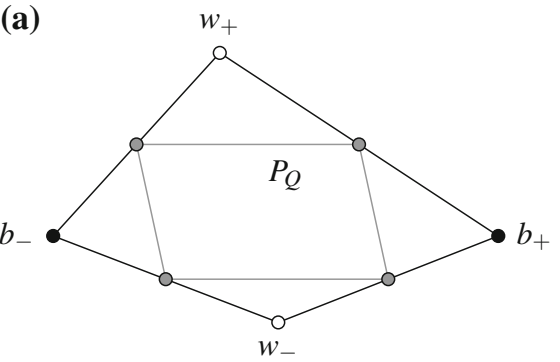

(b)

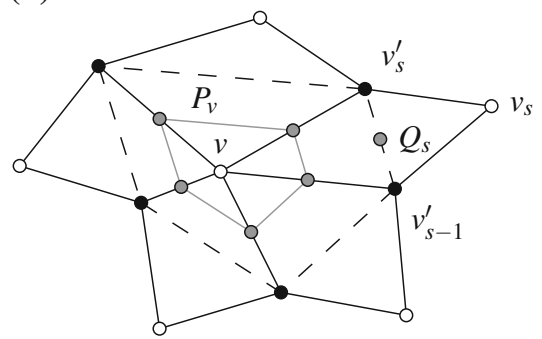

Fig. 3 Illustrations to the integration formulae for discrete derivatives. a Lemma 2.3 for $\partial_{\Lambda}, \bar{\partial}_{\Lambda}$. b Definitions of $\partial_{\diamond}, \bar{\partial} \diamond$ 
Definition $h: V\left(\diamond_{0}\right) \rightarrow \mathbb{C}$ is said to be discrete holomorphic if $h$ is discrete holomorphic at all $v \in V\left(\Lambda_{0}\right) \backslash V\left(\partial \Lambda_{0}\right)$.

Note that in the rhombic case, our definition coincides with the one used by Chelkak and Smirnov in [6]. As an immediate consequence of the definition, we obtain a discrete Morera's theorem.

Proposition 2.4 Functions $f: V\left(\Lambda_{0}\right) \rightarrow \mathbb{C}$ and $h: V\left(\diamond_{0}\right) \rightarrow \mathbb{C}$ are discrete holomorphic if and only if $\oint_{P} f d z=0$ and $\oint_{P} h d z=0$ for all discrete contours $P$ on $X_{0}$.

Proof Clearly, $\oint_{P_{v}} f d z=f(v) \oint_{P_{v}} d z=0$ for any discrete elementary cycle $P_{v}$ around a vertex $v$ of $V\left(\Lambda_{0}\right)$. Similarly, $\oint_{P_{Q}} h d z=0$ for any $Q \in V\left(\diamond_{0}\right)$. Using Lemma 2.3 and the definition of $\bar{\partial}_{\diamond}, f$ and $h$ are discrete holomorphic if and only if $\oint_{P} f d z=0$ and $\oint_{P} h d z=0$ for all discrete elementary cycles $P$. To conclude the proof, we observe that any integration along a discrete contour can be decomposed into integrations along discrete elementary cycles since by definition, a discrete contour is the boundary of a topological disk in $F\left(X_{0}\right)$.

The discrete derivatives of constant functions on $V(\diamond)$ vanish. As an analog of Proposition 2.1 , we prove that the discrete derivatives $\partial_{\diamond}, \bar{\partial} \diamond$ locally approximate their smooth counterparts correctly up to order one if the quadrilaterals in $V(\diamond)$ are identified with the midpoints of their black or white edges. In a parallelogram-graph, these two midpoints coincide for each face, which then gives a global approximation statement. Note that even for rhombic quad-graphs, the discrete derivatives $\partial_{\diamond}, \bar{\partial}_{\diamond}$ generally do not coincide with the smooth derivatives in order two.

Proposition 2.5 Let $v \in V(\Lambda)$, and let $h$ be a complex function on all faces incident to $v$. As illustrated in Fig. $3 b$, we counterclockwise enumerate them by $Q_{1}, \ldots, Q_{k}$, where $k$ is the degree of $v$ in $\Lambda$, and their vertices adjacent to $v$ by $v_{1}^{\prime}, v_{2}^{\prime}, \ldots, v_{k}^{\prime}$, $v_{k+1}^{\prime}=v_{1}$. Let $\hat{Q}_{s}=\left(v_{s-1}^{\prime}+v_{s}^{\prime}\right) / 2$. Then, if $h\left(Q_{s}\right)=\hat{Q}_{s}$ for all $s, \bar{\partial}_{\diamond} h(v)=0$ and $\partial_{\diamond} h(v)=1$ hold true.

\section{Proof}

$$
\begin{aligned}
4 \oint_{P_{v}} h d z & =\sum_{Q_{s} \sim v} 2 h\left(Q_{s}\right)\left(v_{s}^{\prime}-v_{s-1}^{\prime}\right)=\sum_{Q_{s} \sim v}\left(v_{s-1}^{\prime}+v_{s}^{\prime}\right)\left(v_{s}^{\prime}-v_{s-1}^{\prime}\right) \\
& =\sum_{Q_{s} \sim v}\left(\left(v_{s}^{\prime}\right)^{2}-\left(v_{s-1}^{\prime}\right)^{2}\right)=0 \\
4 \oint_{P_{v}} h d \bar{z} & =\sum_{Q_{s} \sim v}\left(v_{s-1}^{\prime}+v_{s}^{\prime}\right) \overline{\left(v_{s}^{\prime}-v_{s-1}^{\prime}\right)}=\sum_{Q_{s} \sim v}\left(\left|v_{s}^{\prime}\right|^{2}-\left|v_{s-1}^{\prime}\right|^{2}-2 i \operatorname{Im}\left(v_{s}^{\prime} \bar{v}_{s-1}^{\prime}\right)\right) \\
& =-2 i \sum_{Q_{s} \sim v} \operatorname{Im}\left(v_{s}^{\prime} \bar{v}_{s-1}^{\prime}\right)=-8 i \operatorname{ar}\left(F_{v}\right) .
\end{aligned}
$$


Thus, $\bar{\partial}_{\diamond} h(v)=0$ and $\partial_{\diamond} h(v)=1$. Here, we have used that by definition, $\operatorname{ar}\left(F_{v}\right)$ is half of the Euclidean area of the polygon $v_{1}^{\prime} v_{2}^{\prime} \ldots v_{k}^{\prime}$, so $\operatorname{ar}(F)$ equals

$$
\frac{1}{2} \sum_{Q_{s} \sim v} \operatorname{area}\left(\Delta v v_{s-1}^{\prime} v_{s}^{\prime}\right)=\frac{1}{4} \sum_{Q_{s} \sim v} \operatorname{Im}\left(\left(v_{s}^{\prime}-v\right) \overline{\left(v_{s-1}^{\prime}-v\right)}\right)=\frac{1}{4} \sum_{Q_{s} \sim v} \operatorname{Im}\left(v_{s}^{\prime} \bar{v}_{s-1}^{\prime}\right),
$$

using that $\sum_{Q_{s} \sim v}\left(v \bar{v}_{s-1}^{\prime}+\bar{v} v_{s}^{\prime}\right)=\sum_{Q_{s} \sim v}\left(v \bar{v}_{s}^{\prime}+\bar{v} v_{s}^{\prime}\right)$ is real.

In [6], Chelkak and Smirnov used averaging operators to map functions on $V(\Lambda)$ to functions on $V(\diamond)$ and vice versa. On parallelogram-graphs, the averaging operator $m(f)(Q):=\sum_{v \sim Q} f(v) / 4$ actually maps discrete holomorphic functions $f$ on $V(\Lambda)$ to discrete holomorphic functions on $V(\diamond)$. Our proof will be similar as the one for rhombic quad-graphs in [6]. Note that discrete holomorphic functions on $V(\diamond)$ cannot be averaged to discrete holomorphic functions on $V(\Lambda)$ in general, so the averaging operator of Chelkak of Smirnov that mapped functions on $V(\diamond)$ to functions on $V(\Lambda)$ did not preserve discrete holomorphicity.

Proposition 2.6 Let $\Lambda$ be a parallelogram-graph and $f: V(\Lambda) \rightarrow \mathbb{C}$ be discrete holomorphic. Then, $m(f): V(\diamond) \rightarrow \mathbb{C}$ is discrete holomorphic.

Proof Let us consider the star of the vertex $v \in V(\Lambda)$ and use the notation we used in Proposition 2.5 (illustrated by Fig. 3b). Since $f$ is discrete holomorphic, the discrete Cauchy-Riemann equation is satisfied on any $Q_{s} \sim v$. Therefore, we can express $f\left(v_{s}\right)$ in terms of $f(v), f\left(v_{s}^{\prime}\right)$ and $f\left(v_{s-1}^{\prime}\right)$. Plugging this in the definition of the averaging operator, we obtain

$$
\begin{aligned}
4 m(f)\left(Q_{s}\right) & =2 f(v)+\frac{v_{s}-v+v_{s}^{\prime}-v_{s-1}^{\prime}}{v_{s}^{\prime}-v_{s-1}^{\prime}} f\left(v_{s}^{\prime}\right)-\frac{v_{s}-v-v_{s}^{\prime}+v_{s-1}^{\prime}}{v_{s}^{\prime}-v_{s-1}^{\prime}} f\left(v_{s-1}^{\prime}\right) \\
& =2 f(v)+2 \frac{v_{s}^{\prime}-v}{v_{s}^{\prime}-v_{s-1}^{\prime}} f\left(v_{s}^{\prime}\right)-2 \frac{v_{s-1}^{\prime}-v}{v_{s}^{\prime}-v_{s-1}^{\prime}} f\left(v_{s-1}^{\prime}\right)
\end{aligned}
$$

Here, we have used the properties $v_{s}-v_{s-1}^{\prime}=v_{s}^{\prime}-v$ and $v_{s}-v_{s}^{\prime}=v_{s-1}^{\prime}-v$ of the parallelogram $Q_{s}$. Hence, $m(f)$ is discrete holomorphic at $v$ by definition due to

$$
4 \oint_{P_{v}} m(f) d z=2 f(v) \oint_{P_{v}} d z+\sum_{Q_{s} \sim v}\left(v_{s}^{\prime}-v\right) f\left(v_{s}^{\prime}\right)-\sum_{Q_{s} \sim v}\left(v_{s-1}^{\prime}-v\right) f\left(v_{s-1}^{\prime}\right)=0 .
$$

Remark As mentioned above, our main interest lies in functions that are defined either on the vertices or the faces of the quad-graph. Now, extending $f: V(\Lambda) \rightarrow \mathbb{C}$ to a complex function on $F(X)$ by using its average $m(f)$ on $V(\diamond)$ seems to be an option. However, functions on $V(\Lambda)$ and on $V(\diamond)$ behave differently. In Corollary 2.11 we will see that $\partial_{\Lambda} f$ is discrete holomorphic if $f$ is, but $\partial_{\diamond} m(f)$ does not need to be discrete holomorphic in general. So to make sense of differentiating twice, we can only consider functions on $V(\Lambda)$. 
Definition Let $f_{1}, f_{2}: V\left(\Lambda_{0}\right) \rightarrow \mathbb{C}$ and $h_{1}, h_{2}: V\left(\diamond_{0}\right) \rightarrow \mathbb{C}$. Their discrete scalar products are defined as

$$
\left\langle f_{1}, f_{2}\right\rangle:=-\frac{1}{2 i} \iint_{F\left(X_{0}\right)} f_{1} \bar{f}_{2} \Omega_{\Lambda} \text { and }\left\langle h_{1}, h_{2}\right\rangle:=-\frac{1}{2 i} \iint_{F\left(X_{0}\right)} h_{1} \bar{h}_{2} \Omega_{\diamond},
$$

whenever the right hand side converges absolutely.

Proposition $2.7-\partial_{\diamond}$ and $-\bar{\partial}_{\diamond}$ are the formal adjoints of $\bar{\partial}_{\Lambda}$ and $\partial_{\Lambda}$, respectively. That is, if $f: V(\Lambda) \rightarrow \mathbb{C}$ or $h: V(\diamond) \rightarrow \mathbb{C}$ is compactly supported, then

$$
\left\langle\partial_{\Lambda} f, h\right\rangle+\left\langle f, \bar{\partial}_{\diamond} h\right\rangle=0=\left\langle\bar{\partial}_{\Lambda} f, h\right\rangle+\left\langle f, \partial_{\diamond} h\right\rangle .
$$

Proof In Lemma 2.3, we showed how the discrete derivative $\partial_{\Lambda} f(Q)$ can be expressed as a contour integration around the face of $X$ associated to $Q \in V(\diamond)$. Using this, the definitions of $\Omega_{\Lambda}$ and $\Omega_{\diamond}$, and $\partial_{\diamond} \bar{h}=\overline{\bar{\partial}} \phi$, we get

$$
\begin{aligned}
\left\langle\partial_{\Lambda} f, h\right\rangle+\langle f, \bar{\partial} \diamond h\rangle & =\sum_{Q \in V(\diamond)} \partial_{\Lambda} f(Q) \bar{h}(Q) \operatorname{ar}\left(F_{Q}\right)+\sum_{v \in V(\Lambda)} f(v) \overline{\bar{\partial}} \bar{\diamond}(v) \operatorname{ar}\left(F_{v}\right) \\
& =\frac{i}{2} \sum_{Q \in V(\diamond)} \bar{h}(Q) \oint_{P_{Q}} f d \bar{z}+\frac{i}{2} \sum_{v \in V(\Lambda)} f(v) \oint_{P_{v}} \bar{h} d \bar{z} \\
& =\frac{i}{2} \oint_{P} f \bar{h} d \bar{z}=0
\end{aligned}
$$

where $P$ is a large contour enclosing all the vertices of $\Lambda$ and $\diamond$ where $f$ or $h$ do not vanish. In particular, $f \bar{h}$ vanishes in a neighborhood of $P$. In the same way, $\left\langle\bar{\partial}_{\Lambda} f, h\right\rangle+\left\langle f, \partial_{\diamond} h\right\rangle=0$.

Remark Note that in their work on discrete complex analysis on rhombic quadgraphs, Kenyon [16] and Mercat [20] did not give explicit formulae for the discrete derivatives, but defined $-\partial_{\diamond}$ and $-\bar{\partial} \diamond$ instead as the formal adjoints of the discrete derivatives $\bar{\partial}_{\Lambda}$ and $\partial_{\Lambda}$, respectively. In contrast, we derived the formal adjoint property from our explicit formulae for the discrete derivatives.

In Corollary 2.11, we will prove that $\partial_{\Lambda} f$ is discrete holomorphic if the function $f: V(\Lambda) \rightarrow \mathbb{C}$ is. Conversely, we can find discrete primitives of discrete holomorphic functions on subgraphs $\nabla_{0} \subseteq \diamond$ that form a simply-connected closed region, extending the corresponding result for rhombic quad-graphs given by Chelkak and Smirnov [6].

Proposition 2.8 Let $\diamond_{0} \subseteq \diamond$ form a simply-connected closed region. Then, for any discrete holomorphic function $h$ on $V\left(\diamond_{0}\right)$, there is a discrete primitive $f:=\int h$ on $V\left(\Lambda_{0}\right)$, i.e., $f$ is discrete holomorphic and $\partial_{\Lambda} f=h . f$ is unique up to two additive constants on $\Gamma_{0}$ and $\Gamma_{0}^{*}$. 
Proof Since $h$ is discrete holomorphic, $\oint_{P} h d z=0$ for any discrete contour $P$ in $X_{0}$ by discrete Morera's Theorem 2.23. Therefore, $h d z$ can be integrated to a welldefined function $f_{X}$ on $V(X)$ that is unique up to an additive constant. The equation $f_{X}((v+w) / 2)=(f(v)+f(w)) / 2$ for any edge $(v, w)$ of $\Lambda$ defines a function $f$ on $V(\Lambda)$. Indeed, since $\nabla_{0}$ forms a simply-connected closed region, it suffices to check the compatibility of the equations defining $f$ just for one quadrilateral face $Q$. Now, the differences of $f$ along the black and white diagonals of $Q$ are given by integration of $h d z$ along the edges of $X$ that are parallel to these diagonals. Since $h d z$ is a discrete one-form of type $\diamond$, the two integrations along the edges of $X$ parallel to one diagonal are the same if they are oriented the same, and compatibility follows. Given $f_{X}, f$ is unique up to another additive constant.

In summary, $f$ is unique up to two additive constants that can be chosen independently on $\Gamma_{0}$ and $\Gamma_{0}^{*}$. By construction, $f$ satisfies

$$
\frac{f\left(b_{+}\right)-f\left(b_{-}\right)}{b_{+}-b_{-}}=h(Q)=\frac{f\left(w_{+}\right)-f\left(w_{-}\right)}{w_{+}-w_{-}}
$$

on any quadrilateral $Q \in V\left(\searrow_{0}\right)$. So $f$ is discrete holomorphic and $\partial_{\Lambda} f=h$.

\subsection{Discrete Exterior Calculus}

Our treatment of discrete exterior calculus is similar to the approach of Mercat in [19-21], but differs in some aspects. The main differences are due to our different notation of multiplication of functions with discrete one-forms, which allows us to define a discrete exterior derivative on a larger class of discrete one-forms in Sect.2.3.1. It coincides with Mercat's discrete exterior derivative in the case of discrete one-forms of type $\diamond$ that Mercat considers. In contrast, our definitions are based on a coordinate representation and mimic the smooth case. Eventually, they lead to essentially the same definitions of a discrete wedge product in Sect.2.3.2 and a discrete Hodge star in Sect. 2.3.3 as in [21].

\subsubsection{Discrete Exterior Derivative}

Definition Let $f: V\left(\Lambda_{0}\right) \rightarrow \mathbb{C}, h: V\left(\diamond_{0}\right) \rightarrow \mathbb{C}$. We define the discrete exterior derivatives $d f$ and $d h$ as the discrete one-forms on oriented edges of $X_{0}$ given by

$$
d f:=\partial_{\Lambda} f d z+\bar{\partial}_{\Lambda} f d \bar{z} \text { and } d h:=\partial_{\diamond} h d z+\bar{\partial}_{\diamond} h d \bar{z}
$$

Definition Let $\omega$ be a discrete one-form defined on all boundary edges of a face $F_{v}$ of the medial graph $X$ corresponding to $v \in V(\Lambda)$ or on all four boundary edges of a face $F_{Q}$ of $X$ corresponding to $Q \in F(\Lambda)$. In the first case, we write $\omega=p d z+q d \bar{z}$ with functions $p, q$ defined on all faces of $\Lambda$ that are incident to $v$, and in the second case, we write $\omega=p d z+q d \bar{z}$ with functions $p, q$ defined on all vertices of $\Lambda$ that are incident to $Q$. The discrete exterior derivative $d \omega$ on $F_{v}$ or $F_{Q}$ is given by 


$$
\left.d \omega\right|_{F_{v}}:=\left(\partial_{\diamond} q-\bar{\partial}_{\diamond} p\right) \Omega_{\Lambda} \text { and }\left.d \omega\right|_{F_{Q}}:=\left(\partial_{\Lambda} q-\bar{\partial}_{\Lambda} p\right) \Omega_{\diamond}
$$

The representation of $\omega$ as $p d z+q d \bar{z}$ ( $p, q$ defined on edges of $X$ ) we have used above may be nonunique. However, $d \omega$ is well-defined by the following discrete Stokes' theorem that also justifies our definition of $d f$ and $d \omega$. Note that Mercat defined the discrete exterior derivative by the discrete Stokes' theorem [19].

Theorem 2.9 Let $f: V\left(\Lambda_{0}\right) \rightarrow \mathbb{C}$ and $\omega$ be a discrete one-form defined on oriented edges of $X_{0}$. Then, for any directed edge e of $X_{0}$ starting in the midpoint of the edge $v v_{-}^{\prime}$ and ending in the midpoint of the edge $v v_{+}^{\prime}$ of $\Lambda_{0}$ and for any finite collection of faces $F$ of $X_{0}$ with counterclockwise oriented boundary $\partial F$ we have:

$$
\int_{e} d f=\frac{f\left(v_{+}^{\prime}\right)-f\left(v_{-}^{\prime}\right)}{2}=\frac{f(v)+f\left(v_{+}^{\prime}\right)}{2}-\frac{f(v)+f\left(v_{-}^{\prime}\right)}{2} \text { and } \iint_{F} d \omega=\oint_{\partial F} \omega .
$$

Proof Let $v_{-}$be the other vertex of the quadrilateral $Q$ with vertices $v, v_{-}^{\prime}$ and $v_{+}^{\prime}$. Without loss of generality, let $v$ be white. Since $d f=\partial_{\Lambda} f d z+\bar{\partial}_{\Lambda} f d \bar{z}, \int_{e} d f$ equals

$$
\begin{aligned}
& \partial_{\Lambda} f \frac{v_{+}^{\prime}-v_{-}^{\prime}}{2}+\bar{\partial}_{\Lambda} f \frac{\overline{v_{+}^{\prime}-v_{-}^{\prime}}}{2} \\
= & \frac{1}{2}\left(\lambda_{Q}+\bar{\lambda}_{Q}\right)\left(f\left(v_{+}^{\prime}\right)-f\left(v_{-}^{\prime}\right)\right)+\frac{1}{2}\left(\bar{\lambda} Q \frac{v_{+}^{\prime}-v_{-}^{\prime}}{v-v_{-}}+\lambda \frac{\overline{v_{+}^{\prime}-v_{-}^{\prime}}}{\overline{v-v_{-}}}\right)\left(f(v)-f\left(v_{-}\right)\right) \\
= & \frac{f\left(v_{+}^{\prime}\right)-f\left(v_{-}^{\prime}\right)}{2}+\operatorname{Re}\left(\bar{\lambda} Q \frac{v_{+}^{\prime}-v_{-}^{\prime}}{v-v_{-}}\right)\left(f(v)-f\left(v_{-}\right)\right)=\frac{f\left(v_{+}^{\prime}\right)-f\left(v_{-}^{\prime}\right)}{2} .
\end{aligned}
$$

To get to the third line, we used $\lambda_{Q}+\bar{\lambda}_{Q}=1$, and for the last step we used

$$
\arg \left(\bar{\lambda}_{Q} \frac{v_{+}^{\prime}-v_{-}^{\prime}}{v-v_{-}}\right)=\arg \left( \pm \exp \left(i\left(\varphi_{Q}-\frac{\pi}{2}\right)\right) \exp \left(-i \varphi_{Q}\right)\right)= \pm \pi / 2
$$

The sign depends on whether $v, v_{-}^{\prime}, v_{-}, v_{+}^{\prime}$ denote the corners of $Q$ in clockwise or counterclockwise order. In either case, the expression inside arg is purely imaginary.

The second identity has to be shown just for one single face of $X_{0}$. Let us write $\omega=p d z+q d \bar{z}$ on all edges of $X_{0}$ that are boundary edges of $F_{Q}$ or $F_{v}$, where $p, q$ are functions defined on the vertices of the quadrilateral $Q \in V\left(\searrow_{0}\right)$ or on the faces incident to $v \in V\left(\Lambda_{0}\right)$. Then, by the representation of $\partial_{\Lambda}, \bar{\partial}_{\Lambda}$ as discrete contour integrals in Lemma 2.3 and the definition of the discrete derivatives $\partial_{\diamond}, \bar{\partial}_{\diamond}$,

$$
\begin{aligned}
& \iint_{F_{Q}} d \omega=\iint_{F_{Q}}\left(\partial_{\diamond} q-\bar{\partial}_{\diamond} p\right) \Omega_{\diamond}=-2 i \operatorname{ar}\left(F_{Q}\right)\left(\partial_{\diamond} q-\bar{\partial}_{\diamond} p\right)=\oint_{\partial F_{Q}}(p d z+q d \bar{z}), \\
& \iint_{F_{v}} d \omega=\iint_{F_{v}}\left(\partial_{\Lambda} q-\bar{\partial}_{\Lambda} p\right) \Omega_{\Lambda}=-2 i \operatorname{ar}\left(F_{v}\right)\left(\partial_{\Lambda} q-\bar{\partial}_{\Lambda} p\right)=\oint_{\partial F_{v}}(p d z+q d \bar{z}) .
\end{aligned}
$$


Definition Let $\nabla_{0} \subseteq \diamond$ form a simply-connected closed region. A discrete one-form $\omega$ defined on oriented edges of $X_{0}$ is said to be closed if $d \omega \equiv 0$.

Note that if $\omega$ is a discrete one-form of type $\diamond$, then $\iint_{F} d \omega=0$ for any face $F$ corresponding to a face of $\Lambda$. Examples for closed discrete one-forms are discrete exterior derivatives of complex functions on $V(\Lambda)$ :

Proposition 2.10 Let $f: V\left(\Lambda_{0}\right) \rightarrow \mathbb{C}$. Then, $d d f=0$ on any face $F_{v}$ of $X_{0}$ corresponding to $v \in V\left(\Lambda_{0}\right) \backslash V\left(\partial \Lambda_{0}\right)$.

Proof By discrete Stokes' Theorem 2.9, we have to show $\oint_{P} d f=0$ for any discrete elementary cycle $P$ in $X_{0}$ in order to prove $d d f=0$. Since $d f$ is of type $\diamond$, the statement is trivially true if $P=P_{Q}$ for $Q \in V\left(\diamond_{0}\right)$. So let $P=P_{v}$ for $v \in V\left(\Lambda_{0}\right) \backslash V\left(\partial \Lambda_{0}\right)$. Using discrete Stokes' Theorem 2.9 again,

$$
\oint_{P_{v}} d f=\sum_{Q_{s} \sim v} \frac{f\left(v_{s}^{\prime}\right)-f\left(v_{s-1}^{\prime}\right)}{2}=0 .
$$

An immediate corollary of the last proposition is the commutativity of discrete derivatives, generalizing the known result for rhombic quad-graphs in [6].

Corollary 2.11 Let $f: V\left(\Lambda_{0}\right) \rightarrow \mathbb{C}$. Then, $\partial_{\diamond} \bar{\partial}_{\Lambda} f(v)=\bar{\partial}_{\diamond} \partial_{\Lambda} f(v)$ for all vertices $v \in V\left(\Lambda_{0}\right) \backslash V\left(\partial \Lambda_{0}\right)$. In particular, $\partial_{\Lambda} f$ is discrete holomorphic if $f$ is discrete holomorphic.

Proof Due to the preceding Proposition 2.10 and the definition of the discrete derivative, the equation $0=d d f=\left(\partial_{\diamond} \bar{\partial}_{\Lambda} f-\bar{\partial}_{\diamond} \partial_{\Lambda} f\right) \Omega_{\Lambda}$ holds on all faces of $X_{0}$ corresponding to a vertex of $\Lambda_{0}$ that is not on the boundary $\partial \Lambda_{0}$. The claim follows since $\Omega_{\Lambda}$ is nonzero on these faces.

Remark Note that even in the generic rhombic case, $\partial_{\Lambda} \bar{\partial}_{\diamond} h$ does not always equal $\bar{\partial}_{\Lambda} \partial_{\diamond} h$ for a function $h: V(\diamond) \rightarrow \mathbb{C}[6]$. Hence, $d d h=0$ cannot hold for such functions $h$ in general.

Corollary 2.12 Let $f: V\left(\Lambda_{0}\right) \rightarrow \mathbb{C}$. Then, $f$ is discrete holomorphic if and only if $d f=p d z$ for some $p: V\left(\diamond_{0}\right) \rightarrow \mathbb{C}$. In the case that $f$ is discrete holomorphic, $p$ is discrete holomorphic as well.

Proof Since all quadrilaterals $Q \in V\left(\diamond_{0}\right)$ are nondegenerate, the representation of $\left.d f\right|_{\partial F_{Q}}$ as $p d z+q d \bar{z}$ is unique (see Lemma 2.14 below). Clearly, we have $d f=\partial_{\Lambda} f d z+\bar{\partial}_{\Lambda} f d \bar{z}$. It follows that $f$ is discrete holomorphic at $Q$ if and only if $\left.d f\right|_{\partial F_{Q}}=p d z$.

Assuming that $d f=p d z$ for some $p: V\left(\diamond_{0}\right) \rightarrow \mathbb{C}, d d f=0$ on any face $F_{v}$ of $X_{0}$ corresponding to $v \in V\left(\Lambda_{0}\right) \backslash V\left(\partial \Lambda_{0}\right)$ by Proposition 2.10. Thus, $\bar{\partial} \diamond p(v)=0$ for any such $v$ and $f$ is discrete holomorphic.

Definition Let $\nabla_{0} \subseteq \diamond$ form a simply-connected closed region. A discrete one-form $\omega$ defined on the oriented edges of $X_{0}$ is discrete holomorphic if $\omega=p d z$ for some $p: V\left(\diamond_{0}\right) \rightarrow \mathbb{C}$ and $d \omega=0$. 
Remark This notion recurs in the more general setting of discrete Riemann surfaces in [1]. By Corollary 2.12, $d f$ is discrete holomorphic if $f$ is, and by Proposition 2.8 on the existence of a discrete primitive for discrete holomorphic functions defined on the vertices of a subset $\nabla_{0} \subseteq \diamond$ that forms a simply-connected closed region, any discrete holomorphic one-form $\omega$ defined on the oriented edges of $X_{0}$ is the discrete exterior derivative of a discrete holomorphic function on $V\left(\Lambda_{0}\right)$.

Due to Chelkak and Smirnov [6], one of the unpleasant facts of all discrete theories of complex analysis is that (pointwise) multiplication of discrete holomorphic functions does not yield a discrete holomorphic function in general. We define a product of complex functions on $V(\Lambda)$ that is defined on $V(X)$ and a product of complex functions on $V(\Lambda)$ with functions on $V(\diamond)$ that is defined on $E(X)$. In general, the product of two discrete holomorphic functions is not discrete holomorphic according to the classical quad-based definition (on planar quad-graphs different from $\Lambda$ ), but it will be discrete holomorphic in the sense that a discretization of its exterior derivative is closed and is of the form $p d z, p$ defined on the edges of the medial graph of the new quad-graph, or in the sense that it fulfills a discrete Morera's theorem.

Proposition 2.13 Let $f, g: V(\Lambda) \rightarrow \mathbb{C}$ and $h: V(\diamond) \rightarrow \mathbb{C}$.

(i) $f d g+g d f$ is a closed discrete one-form.

(ii) If $f$ and $h$ are discrete holomorphic, then $f$ hdz is a closed discrete one-form.

Proof (i) Let $\omega:=f d g+g d f$. By Proposition 2.10, $d d f=0$ and $d d g=0$, i.e., $d f$ and $d g$ are closed. Thus,

$$
\oint_{\partial F_{v}} \omega=f(v) \oint_{\partial F_{v}} d g+g(v) \oint_{\partial F_{v}} d f=0
$$

for any face $F_{v}$ corresponding to $v \in V(\Lambda)$. Using Lemma 2.3 that relates discrete derivatives with discrete contour integration,

$$
\begin{aligned}
2 i \operatorname{ar}\left(F_{Q}\right) \oint_{\partial F_{Q}} \omega & =2 i \operatorname{ar}\left(F_{Q}\right) \oint_{\partial F_{Q}}\left(f \partial_{\Lambda} g d z+f \bar{\partial}_{\Lambda} g d \bar{z}+g \partial_{\Lambda} f d z+g \bar{\partial}_{\Lambda} f d \bar{z}\right) \\
& =\left(\bar{\partial}_{\Lambda} f \partial_{\Lambda} g-\partial_{\Lambda} f \bar{\partial}_{\Lambda} g+\bar{\partial}_{\Lambda} g \partial_{\Lambda} f-\partial_{\Lambda} g \bar{\partial}_{\Lambda} f\right)(Q)=0
\end{aligned}
$$

for any face $F_{Q}$ corresponding to $Q \in F(\Lambda)$. It follows by discrete Stokes' Theorem 2.9 that $d \omega=0$.

(ii) By discrete Morera's Theorem 2.23, discrete holomorphicity of $f$ and $h$ implies that $f d z$ and $h d z$ are closed. Thus, $\oint_{\partial F_{v}} f h d z=f(v) \oint_{\partial F_{v}} h d z=0$ as well as $\oint_{\partial F_{Q}} f h d z=h(Q) \oint_{\partial F_{Q}} f d z=0$ for any faces $F_{v}$ and $F_{Q}$ of $X$ corresponding to $v \in V(\Lambda)$ and $Q \in F(\Lambda)$. Therefore, $f h d z$ is closed.

Remark In particular, for any $f, g: V(\Lambda) \rightarrow \mathbb{C}$ a product $f \cdot g: V(X) \rightarrow \mathbb{C}$ can be defined by integration of $f d g+g d f$. Note that this product $f \cdot g$ is defined up to 
an additive constant. Furthermore, $f \cdot h: E(X) \rightarrow \mathbb{C}$ can be defined by "pointwise" multiplication. If $f, g, h$ are discrete holomorphic, then $f d g+g d f=p d z$ is closed, where $p=f \cdot \partial_{\Lambda} g+g \cdot \partial_{\Lambda} f: E(X) \rightarrow \mathbb{C}$, and so to say a discrete holomorphic one-form, meaning that $f \cdot g$ is discrete holomorphic in this sense. Similarly, $f h d z$ is closed, so $f \cdot h$ is discrete holomorphic in the sense that a discrete Morera's theorem holds true. Even though $f \cdot g$ is defined on the vertices of the dual of a planar quadgraph different from $\Lambda$, as well is $f \cdot h$ on the dual of a different planar quad-graph, these products are generally not discrete holomorphic everywhere according to the quad-based definition of discrete holomorphicity on the dual of a bipartite quad-graph given by the definition in Sect.2.2.3. To define the mentioned planar quad-graphs, we identify $Q \in V(\diamond)$ with such a point in the interior of the face $Q$ that all line segments connecting it to the four corners of $Q$ lie inside the quadrilateral.

First, $f \cdot g$ is a complex function on the vertices of $X$. The medial graph $X$ is the dual of the bipartite quad-graph with vertex set $V(\Lambda) \sqcup V(\diamond)$, edges connecting points $Q \in V(\diamond)$ with all incident vertices $v \in V(\Lambda)$, and faces in one-to-one correspondence to edges of $\Lambda$. But even if $f$ and $g$ are discrete holomorphic on $V(\Lambda)$, $f \cdot g$ does not need to be a discrete holomorphic function on the faces of the quadgraph we just defined. For example, consider $f(v)=0$ if $v$ is black and $f(v)=1$ if $v$ is white and a discrete holomorphic $g$ that is not biconstant. Then, the product $f \cdot g$ is not discrete holomorphic at all $Q \in V(\diamond)$ (seen as vertices of the quad-graph described above) where $\partial_{\Lambda} g(Q) \neq 0$.

Second, $f \cdot h$ is a complex function on the edges of $X$, so it is a function on the vertices of the medial graph of $X$. The medial graph of the medial graph of $\Lambda$ is the dual of the quad-graph with vertex set $(V(\Lambda) \cup V(\diamond)) \sqcup V(X)$, edges connecting points $v \in V(\Lambda)$ or $Q \in V(\diamond)$ with the midpoints of all incident edges, and each face being in one-to-one correspondence to an edge of $X$. Since $f h d z$ is closed, $f \cdot h$ is discrete holomorphic on the new quad-graph at vertices of $\Lambda$ or $\diamond$ by discrete Morera's Theorem 2.23. But there is no need for $f \cdot h$ to be discrete holomorphic at vertices of $X$, even for constant $h$. For example, take the function $f$ defined by $f(v)=0$ if $v$ is black and $f(v)=1$ if $v$ is white.

In summary, we defined products $f \cdot g$ and $f \cdot h$, where $f, g: V(\Lambda) \rightarrow \mathbb{C}$ and $h: V(\diamond) \rightarrow \mathbb{C}$ are discrete holomorphic, that are local (on each vertex, they depend just on the values of $f$ and $g$ in a small neighborhood) and discrete holomorphic at least in the sense that its discrete exterior derivative is closed and of the form $p d z$ or in the sense that it fulfills a discrete Morera's theorem.

Somehow missing is a product $h \cdot h^{\prime}$, where $h, h^{\prime}: V(\diamond) \rightarrow \mathbb{C}$. In the general case, we do not know an appropriate product so far. But we want to point out that Chelkak and Smirnov showed in [7] that for so-called spin holomorphic functions $h, h^{\prime}$, the pointwise product satisfies $\operatorname{Re}\left(\bar{\partial}_{\diamond}\left(h \cdot h^{\prime}\right)\right) \equiv 0$.

\subsubsection{Discrete Wedge Product}

Following Whitney [27], Mercat defined in [19] a discrete wedge product for discrete one-forms living on the edges of $\Lambda$. Then, the discrete exterior derivative defined by 
a discretization of Stokes' theorem is a derivation for the discrete wedge product. However, a discrete Hodge star cannot be defined on $\Lambda$. To circumvent this problem, Mercat used an averaging map to relate discrete one-forms on the edges of $\Lambda$ with discrete one-forms on the edges of $\Gamma$ and $\Gamma^{*}$, i.e., discrete one-forms of type $\nabla$. Then, he could define a discrete Hodge star; however, the discrete exterior derivative was not a derivation for the now heterogeneous discrete wedge product.

In this section, a different interpretation of the discrete wedge product is proposed. Still, the notions of Mercat in [19-21] are recovered. Starting with discrete one-forms of type $\diamond$ that are defined on the edges of $X$, a discrete wedge product on (half of) the faces of $X$ is defined. This definition is different from Whitney's [27] and has the advantage that both a discrete wedge product and a discrete Hodge star can be defined on the same structure. In addition, the discrete exterior derivative is now a derivation for the discrete wedge product in a well-defined sense, see Theorem 2.16. It turns out that Theorem 2.16 is a powerful tool leading to discretizations of Green's identities in Sect. 2.4.1 and of a Cauchy's integral formula for the discrete derivative of a discrete holomorphic function in Sect.2.6.

Lemma 2.14 Let $\omega$ be a discrete one-form of type $\diamond$ defined on the oriented edges of $X_{0}$. Then, there is a unique representation $\omega=p d z+q d \bar{z}$ with $p, q: V\left(\diamond_{0}\right) \rightarrow \mathbb{C}$. On a quadrilateral $Q \in V\left(\diamond_{0}\right), p$ and $q$ are given by

$$
p(Q)=\lambda_{Q} \frac{\int_{e} \omega}{e}+\bar{\lambda} \frac{\int_{e^{*}} \omega}{e^{*}} \text { and } q(Q)=\bar{\lambda}_{Q} \frac{\int_{e} \omega}{\bar{e}}+\lambda_{Q} \frac{\int_{e^{*}} \omega}{\bar{e}^{*}} .
$$

Here, $e$ is an oriented edge of $X_{0}$ parallel to a black edge of $\Gamma_{0}$, and $e^{*}$ is parallel to a white edge of $\Gamma_{0}^{*}$.

Proof First, we show that a representation $\left.\omega\right|_{\partial F_{Q}}=p d z+q d \bar{z}$ exists for any face $F_{Q}$ of $X_{0}$ corresponding to a quadrilateral $Q \in V\left(\nabla_{0}\right)$. Given $\omega$, we have to solve the system of linear equations $\int_{e_{Q}} \omega=p \int_{e_{Q}} d z+q \int_{e_{Q}} d \bar{z}$ for all four boundary edges $e_{Q}$ of $F_{Q}$. Since $\omega$ is of type $\diamond$, we just have to consider two equations, namely one for a boundary edge $e_{b}$ of $F_{Q}$ parallel to a black edge of $\Gamma_{0}$ and one equation for a boundary edge $e_{w}$ parallel to a white edge of $\Gamma_{0}^{*}$. Since all quadrilaterals are nondegenerate, the diagonals are not parallel to each other and it follows that the pair $(d z, d \bar{z})$ gives different values when integrated over $e_{b}$ and $e_{w}$. Thus, this system of two linear equations in two variables is nondegenerate. It follows that $p, q$ are uniquely defined on $V\left(\diamond_{0}\right)$.

Furthermore, we can find for any quadrilateral $Q \in V\left(\diamond_{0}\right) \cong F\left(\Lambda_{0}\right)$ a function $f$ that is defined on the vertices $b_{ \pm}, w_{ \pm}$of $Q$ such that $2 \int_{e} \omega=f\left(b_{+}\right)-f\left(b_{-}\right)$and $2 \int_{e^{*}} \omega=f\left(w_{+}\right)-f\left(w_{-}\right)$, where $e$ is one of the two oriented edges of $X_{0}$ going from the midpoint of $b_{-}$and $w_{ \pm}$to the midpoint of $b_{+}$and $w_{ \pm}$, and $e^{*}$ is one of the two edges connecting the midpoint of $w_{-}$and $b_{ \pm}$with the midpoint of $w_{+}$and $b_{ \pm}$. By discrete Stokes' Theorem 2.9, we get $\left.\omega\right|_{\partial F_{Q}}=d f=p d z+q d \bar{z}$ with $p=\partial_{\Lambda} f(Q)$ and $q=\bar{\partial}_{\Lambda} f(Q)$. Replacing the differences of $f$ in the definition of the discrete derivative by discrete integrals of $\omega$ yields the desired result. 
Definition Let $\omega=p d z+q d \bar{z}$ and $\omega^{\prime}=p^{\prime} d z+q^{\prime} d \bar{z}$ be two discrete one-forms of type $\diamond$ defined on the oriented edges of $X_{0}$. Here, $p, p^{\prime}, q, q^{\prime}: V\left(\diamond_{0}\right) \rightarrow \mathbb{C}$ are given by the above Lemma 2.14. Then, the discrete wedge product $\omega \wedge \omega^{\prime}$ is defined as the discrete two-form of type $\diamond$ defined on $F\left(X_{0}\right)$ that equals

$$
\left(p q^{\prime}-q p^{\prime}\right) \Omega_{\diamond}
$$

on faces of $X$ corresponding to interior faces of the quad-graph $\Lambda_{0}$.

Remark Note that if one considers $d z$ and $d \bar{z}$ as discrete one-forms of type $\diamond$, then $\Omega_{\diamond}=d z \wedge d \bar{z}$.

Proposition 2.15 Let $F$ be a face of $X_{0}$ corresponding to $Q \in F\left(\Lambda_{0}\right)$, and let e, $e^{*}$ be oriented edges of $X$ parallel to the black and white diagonal of the quadrilateral $Q$, respectively, such that $\operatorname{Im}\left(e^{*} / e\right)>0$. If $\omega, \omega^{\prime}$ are discrete one-forms of type $\diamond$ defined on the oriented edges of $\partial F$, then

$$
\iint_{F} \omega \wedge \omega^{\prime}=2 \int_{e} \omega \int_{e^{*}} \omega^{\prime}-2 \int_{e^{*}} \omega \int_{e} \omega^{\prime} .
$$

Proof Both sides of the equation are bilinear and antisymmetric in $\omega, \omega^{\prime}$. Hence, it suffices to check the identity for $\omega=d z, \omega^{\prime}=d \bar{z}$. On the left hand side, we get $\iint_{F} \omega \wedge \omega^{\prime}=-2 i \operatorname{ar}(F)$. This equals the right hand side

$$
2 e \bar{e}^{*}-2 e^{*} \bar{e}=4 i \operatorname{Im}\left(e \bar{e}^{*}\right)=-i|2 e|\left|2 e^{*}\right| \sin \left(\varphi_{Q}\right)=-2 i \operatorname{ar}(F) .
$$

Remark Since the complex numbers $e$ and $e^{*}$ are just half of the oriented diagonals, the above definition of the discrete wedge product is essentially the same as the one given by Mercat in [19-21].

The discrete exterior derivative is a derivation for the discrete wedge product if one considers functions on $\Lambda$ and discrete one-forms of type $\diamond$ :

Theorem 2.16 Let $f: V\left(\Lambda_{0}\right) \rightarrow \mathbb{C}$ and $\omega$ be a discrete one-form of type $\diamond$ defined on the oriented edges of $X_{0}$. Then, the following identity holds on $F\left(X_{0}\right)$ :

$$
d(f \omega)=d f \wedge \omega+f d \omega .
$$

Proof Let $\omega=p d z+q d \bar{z}$ with $p, q: V\left(\diamond_{0}\right) \rightarrow \mathbb{C}$ given by Lemma 2.14. If $F_{v}$ and $F_{Q}$ are faces of $X_{0}$ corresponding to a vertex $v$ and a face $Q$ of $\Lambda_{0}$, then

$$
\begin{aligned}
\left.d(f \omega)\right|_{F_{v}} & =\left(f(v)\left(\partial_{\diamond} q\right)(v)-f(v)\left(\bar{\partial}_{\diamond} p\right)(v)\right) \Omega_{\Lambda}=\left.f d \omega\right|_{F_{v}}, \\
\left.d(f \omega)\right|_{F_{Q}} & =\left(q(Q)\left(\partial_{\Lambda} f\right)(Q)-p(Q)\left(\bar{\partial}_{\Lambda} f\right)(Q)\right) \Omega_{\diamond}=\left.(d f \wedge \omega)\right|_{F_{Q}} .
\end{aligned}
$$


But $\left.(d f \wedge \omega)\right|_{F_{v}}=0$ since $\left.\Omega_{\diamond}\right|_{F_{v}}=0$ and $\left.f d \omega\right|_{F_{Q}}=0$ since $\omega$ is of type $\diamond$, so $d(f \omega)=d f \wedge \omega+f d \omega$.

Remark In [19], Mercat formulated an analog of the above Theorem 2.16 in a setting where discrete one-forms are defined on edges of $\Lambda$. In the setting of discrete oneforms defined on edges of $\Gamma$ and $\Gamma^{*}$, the claim $d(f \omega)=d f \wedge \omega+f d \omega$ could not be well-defined.

Above, a discrete wedge product just of two discrete one-forms of type $\diamond$ is defined. Actually, we could define a discrete wedge product of two discrete oneforms of type $\Lambda$ in essentially the same way, getting a discrete two-form of type $\Lambda$. Then, the analog of Theorem 2.16 would be true for this kind of discrete wedge product and functions on $V\left(\diamond_{0}\right)$. Also the discrete Hodge star of a discrete one-form in the next section could be defined not only for those of type $\diamond$. However, there exist no analogs of Propositions 2.15 and 2.18. These propositions imply that the discrete wedge product as well as the discrete Hodge star of discrete one-forms of type $\diamond$ can be defined in a chart-independent way. This enables one to consider these objects on discrete Riemann surfaces, see [1]. There are no such statements if one chooses discrete one-forms of type $\Lambda$. In fact, a discrete one-form of type $\Lambda$ cannot be canonically defined on a discrete Riemann surface as opposed to discrete one-forms of type $\diamond$. So since our interest lies in the latter, we do not define a discrete wedge product or a discrete Hodge star for discrete one-forms of type $\Lambda$.

\subsubsection{Discrete Hodge Star}

Definition Let $f: F\left(\Lambda_{0}\right) \rightarrow \mathbb{C}$ and $h: V\left(\diamond_{0}\right) \rightarrow \mathbb{C}$, let $\omega=p d z+q d \bar{z}$ be a discrete one-form of type $\diamond$ defined on oriented edges of $X_{0}$ with $p, q: V\left(\diamond_{0}\right) \rightarrow \mathbb{C}$, and let $\Omega_{1}, \Omega_{2}: F\left(X_{0}\right) \rightarrow \mathbb{C}$ be discrete two-forms of type $\Lambda$ and $\diamond$. Then, the discrete Hodge star is given by

$$
\begin{aligned}
\star f:=-\frac{1}{2 i} f \Omega_{\Lambda} ; \quad \star h:=-\frac{1}{2 i} h \Omega_{\diamond} ; \quad \star \omega:=-i p d z+i q d \bar{z} ; \\
\star \Omega_{1}:=-2 i \frac{\Omega_{1}}{\Omega_{\Lambda}} ; \quad \star \Omega_{2}:=-2 i \frac{\Omega_{2}}{\Omega_{\diamond}} .
\end{aligned}
$$

If $\omega$ and $\omega^{\prime}$ are both discrete one-forms of type $\diamond$ defined on oriented edges of $X_{0}$, we define their discrete scalar product

$$
\left\langle\omega, \omega^{\prime}\right\rangle:=\iint_{F\left(X_{0}\right)} \omega \wedge \star \bar{\omega}^{\prime},
$$

whenever the right hand side converges absolutely. Similarly, a discrete scalar product for discrete two-forms of the same type is defined. 
Remark As in the classical theory, the Hodge star corresponds to a $\pi / 2$-rotation: $\int_{i e} \star \omega=\int_{e} \omega$ where $\omega$ is a discrete one-form of type $\diamond, e$ an oriented edge of $X$ and $i e$ its (virtual) image under $\pi / 2$-rotation around the origin.

Corollary 2.17 The following statements are true:

(i) $\star^{2}=-I d$ on discrete one-forms of type $\diamond$ defined on oriented edges of $X_{0}$.

(ii) $\star^{2}=$ Id on complex functions on $V\left(\Lambda_{0}\right)$ or $V\left(\diamond_{0}\right)$ and discrete two-forms defined on $F\left(X_{0}\right)$ of type $\Lambda$ or $\diamond$.

(iii) $f: V\left(\Lambda_{0}\right) \rightarrow \mathbb{C}$ is discrete holomorphic if and only if $\star d f=-i d f$.

(iv) $\left\langle f_{1}, f_{2}\right\rangle=\iint_{F\left(X_{0}\right)} f_{1} \overline{\star f_{2}}$ for functions $f_{1}, f_{2}: V\left(\Lambda_{0}\right) \rightarrow \mathbb{C}$ and $\left\langle h_{1}, h_{2}\right\rangle=\iint_{F\left(X_{0}\right)} h_{1} \overline{\star h_{2}}$ for functions $h_{1}, h_{2}: V\left(\diamond_{0}\right) \rightarrow \mathbb{C}$.

(v) $\langle\cdot, \cdot\rangle$ is a Hermitian scalar product on discrete differential forms (of type $\Lambda$ or of type $\diamond)$.

Proposition 2.18 Let $Q \in V(\diamond)$, and let $e, e^{*}$ be oriented edges of $X$ parallel to the black and white diagonal of $Q$, respectively, such that $\operatorname{Im}\left(e^{*} / e\right)>0$. If $\omega$ is a discrete one-form of type $\diamond$ defined on the oriented edges of the boundary of the face of $X$ corresponding to $Q$, then

$$
\begin{aligned}
& \int_{e} \star \omega=\cot \left(\varphi_{Q}\right) \int_{e} \omega-\frac{|e|}{\left|e^{*}\right| \sin \left(\varphi_{Q}\right)} \int_{e^{*}} \omega, \\
& \int_{e^{*}} \star \omega=\frac{\left|e^{*}\right|}{|e| \sin \left(\varphi_{Q}\right)} \int_{e} \omega-\cot \left(\varphi_{Q}\right) \int_{e^{*}} \omega .
\end{aligned}
$$

Proof Both sides of any of the two equations are linear and behave the same under complex conjugation. Thus, it suffices to check the statement for $\omega=d z$. Hence, it remains to show that

$$
-i e=\cot \left(\varphi_{Q}\right) e-\frac{|e|}{\left|e^{*}\right| \sin \left(\varphi_{Q}\right)} e^{*} \text { and } e^{*}=\frac{\left|e^{*}\right|}{|e| \sin \left(\varphi_{Q}\right)} e-\cot \left(\varphi_{Q}\right) e^{*} .
$$

Now, both sides of the first equation behave the same under scaling and simultaneous rotation of $e$ and $e^{*}$, the same statement is true for the second equation. Thus, we may assume $e=1$ and $e^{*}=\cos \left(\varphi_{Q}\right)+i \sin \left(\varphi_{Q}\right)$. Multiplying both equations by $\sin \left(\varphi_{Q}\right)$ gives the equivalent statements

$$
\begin{aligned}
-i \sin \left(\varphi_{Q}\right) & =\cos \left(\varphi_{Q}\right)-\left(\cos \left(\varphi_{Q}\right)+i \sin \left(\varphi_{Q}\right)\right), \\
-i \sin \left(\varphi_{Q}\right) \exp \left(i \varphi_{Q}\right) & =1-\cos \left(\varphi_{Q}\right) \exp \left(i \varphi_{Q}\right) .
\end{aligned}
$$

Both equations are true, noting that $\cos \left(\varphi_{Q}\right)-i \sin \left(\varphi_{Q}\right)=\exp \left(-i \varphi_{Q}\right)$.

Remark Proposition 2.18 shows that our definition of a discrete Hodge star on discrete one-forms coincides with Mercat's definition given in [21]. But on discrete 
two-forms and complex functions, our definition of the discrete Hodge star includes an additional factor of the area of the corresponding face of $X$.

Proposition $2.19 \delta:=-\star d \star$ is the formal adjoint of the discrete exterior derivative $d:$ Let $f: V(\Lambda) \rightarrow \mathbb{C}$, and let $\omega$ be a discrete one-form of type $\diamond$ defined on the oriented edges of $X$ and $\Omega: F(X) \rightarrow \mathbb{C}$ a discrete two-form of type $\Lambda$. Assume that all of them are compactly supported. Then,

$$
\langle d f, \omega\rangle=\langle f, \delta \omega\rangle \text { and }\langle d \omega, \Omega\rangle=\langle\omega, \delta \Omega\rangle .
$$

Proof By the assumption that all forms are compactly supported, we can take a large enough finite $\nabla_{0} \subseteq \diamond$ that forms a simply-connected closed region such that $f, \omega, \Omega$ vanish outside $\Lambda_{0}, X_{0}, \diamond_{0}$ and $\omega$ is zero on the boundary $\partial X_{0}$. By discrete Stokes' Theorem 2.9 and Theorem 2.16 that states that the discrete exterior derivative is a derivation for the discrete wedge product,

$$
\begin{aligned}
& 0=\oint_{\partial X_{0}} f \star \bar{\omega}=\iint_{F\left(X_{0}\right)} d(f \star \bar{\omega})=\iint_{F\left(X_{0}\right)} f d \star \bar{\omega}+\iint_{F\left(X_{0}\right)} d f \wedge \star \bar{\omega}=\langle f, \star d \star \omega\rangle+\langle d f, \omega\rangle, \\
& 0=\oint_{\partial X_{0}} \star \bar{\Omega} \omega=\iint_{F\left(X_{0}\right)} d(\star \bar{\Omega} \omega)=\iint_{F\left(X_{0}\right)} \star \bar{\Omega} d \omega+\iint_{F\left(X_{0}\right)}(d \star \bar{\Omega}) \wedge \omega=\langle d \omega, \Omega\rangle-\langle\omega, \delta \Omega\rangle .
\end{aligned}
$$

In the last equalities, we have used Corollary 2.17(ii) and (iv) (the basic properties of the discrete Hodge star) and the observation that complex conjugation commutes with the discrete Hodge star and the discrete exterior derivative. The latter observation immediately follows from the definitions that mimic the classical theory.

\subsection{Discrete Laplacian}

The discrete Laplacian and the discrete Dirichlet energy on general quad-graphs were introduced by Mercat in [21]. Later, Skopenkov reintroduced these definitions in [23], taking the same definition in a different notation. In our discussion of the discrete Laplacian in Sect.2.4.1, we follow the classical approach of Mercat (up to sign) and adapt it to our notations. A feature of the medial graph approach is that it allows to formulate a discrete analog of Green's first identity from which discrete Green's second identity immediately follows.

In Sect.2.4.2, the discrete Dirichlet energy is investigated. In particular, in Theorem 2.30 it is shown how uniqueness and existence of solutions to the discrete Dirichlet boundary value problem imply surjectivity of the discrete derivatives and the discrete Laplacian. We conclude this section with a result concerning the asymptotics of discrete harmonic functions. 


\subsubsection{Definition and Basic Properties}

Definition The discrete Laplacian on functions $f: V(\Lambda) \rightarrow \mathbb{C}$, discrete one-forms of type $\diamond$, or discrete two-forms of type $\Lambda$ is defined as the linear operator

$$
\triangle:=-\delta d-d \delta=\star d \star d+d \star d \star .
$$

For a connected subgraph $\nabla_{0} \subseteq \diamond$ and $f: V\left(\Lambda_{0}\right) \rightarrow \mathbb{C}, \triangle f$ is still defined by the formula above as a complex function on $V\left(\Lambda_{0}\right) \backslash V\left(\partial \Lambda_{0}\right) . f$ is said to be discrete harmonic at $v \in V\left(\Lambda_{0}\right) \backslash V\left(\partial \Lambda_{0}\right)$ if $\triangle f(v)=0 . f$ is discrete harmonic if it is discrete harmonic at all such $v$.

The following factorization of the discrete Laplacian in terms of discrete derivatives generalizes the corresponding result given by Chelkak and Smirnov in [6] to general quad-graphs. The local representation of $\Delta f$ at $v \in V(\Lambda)$ is, up to a factor involving the area of the face $F_{v}$ of $X$ corresponding to $v$, the same as Mercat's [21].

Corollary 2.20 Let $f: V\left(\Lambda_{0}\right) \rightarrow \mathbb{C}$. Then, $\triangle f(v)=4 \partial_{\diamond} \bar{\partial}_{\Lambda} f(v)=4 \bar{\partial}_{\diamond} \partial_{\Lambda} f(v)$ for all vertices $v \in V\left(\Lambda_{0}\right) \backslash V\left(\partial \Lambda_{0}\right)$ and

$$
\Delta f(v)=\frac{1}{2 a r\left(F_{v}\right)} \sum_{Q_{s} \sim v} \frac{1}{\operatorname{Re}\left(\rho_{s}\right)}\left(\left|\rho_{s}\right|^{2}\left(f\left(v_{s}\right)-f(v)\right)+\operatorname{Im}\left(\rho_{s}\right)\left(f\left(v_{s}^{\prime}\right)-f\left(v_{s-1}^{\prime}\right)\right)\right) .
$$

Here, $\rho_{s}:=\rho_{Q_{s}}$ if $v$ is black, and $\rho_{s}:=1 / \rho_{Q_{s}}$ if $v$ is white.

In particular, $\operatorname{Re}(\triangle f) \equiv \triangle \operatorname{Re}(f)$ and $\operatorname{Im}(\triangle f) \equiv \triangle \operatorname{Im}(f)$.

Proof Since the definitions of the discrete Hodge star and the discrete exterior derivative mimic the classical theory and $\partial_{\diamond} \bar{\partial}_{\Lambda} f(v)=\bar{\partial}_{\diamond} \partial_{\Lambda} f(v)$ by Corollary 2.11,

$$
\triangle f(v)=\star d \star d f(v)=2 \partial_{\diamond} \bar{\partial}_{\Lambda} f(v)+2 \bar{\partial}_{\diamond} \partial_{\Lambda} f(v)=4 \partial_{\diamond} \bar{\partial}_{\Lambda} f(v)=4 \bar{\partial}_{\diamond} \partial_{\Lambda} f(v)
$$

holds exactly as in the smooth setting.

For the second statement, let us assume without loss of generality that $v \in V\left(\Gamma_{0}\right)$. Then, we have to show that

$$
\Delta f(v)=\frac{1}{2 \operatorname{ar}\left(F_{v}\right)} \sum_{Q_{s} \sim v}\left(\frac{\left|\rho_{Q_{s}}\right|}{\sin \left(\varphi_{Q_{s}}\right)}\left(f\left(v_{s}\right)-f(v)\right)-\cot \left(\varphi_{Q_{s}}\right)\left(f\left(v_{s}^{\prime}\right)-f\left(v_{s-1}^{\prime}\right)\right)\right) .
$$

The structure is similar to the formula of the discrete Hodge star in Proposition 2.18. Indeed, if $e_{s}$ denotes an edge of $X$ parallel to the black diagonal $v v_{s}$ and $e_{s}^{*}$ an edge parallel to the dual diagonal, then 


$$
\begin{aligned}
\Delta f(v) & =\frac{1}{\operatorname{ar}\left(F_{v}\right)} \iint_{F_{v}} d \star d f=\frac{1}{\operatorname{ar}\left(F_{v}\right)} \oint_{\partial F_{v}} \star d f \\
& =\frac{1}{\operatorname{ar}\left(F_{v}\right)} \sum_{Q_{s} \sim v}\left(\frac{\left|e_{s}^{*}\right|}{\left|e_{s}\right| \sin \left(\varphi_{Q_{s}}\right)} \int_{e_{s}} d f-\cot \left(\varphi_{Q_{s}}\right) \int_{e_{s}^{*}} d f\right) \\
& =\frac{1}{2 \operatorname{ar}\left(F_{v}\right)} \sum_{Q_{s} \sim v}\left(\frac{\left|\rho_{Q_{s}}\right|}{\sin \left(\varphi_{Q_{s}}\right)}\left(f\left(v_{s}\right)-f(v)\right)-\cot \left(\varphi_{Q_{s}}\right)\left(f\left(v_{s}^{\prime}\right)-f\left(v_{s-1}^{\prime}\right)\right)\right),
\end{aligned}
$$

using discrete Stokes' Theorem 2.9 in the first and third equality, Proposition 2.18 that compares the integration of the discrete Hodge star of a discrete one-form of type $\diamond$ with the integration of the discrete one-form $d f$ itself in the second equality, and $\left|\rho_{Q_{s}}\right|=\left|e_{s}^{*}\right| /\left|e_{s}\right|$ for the last step.

Remark In the case when the diagonals of the quadrilaterals are orthogonal to each other, $\rho_{Q}$ is always a positive real number. In this case, the discrete Laplacian splits into two separate discrete Laplacians on $\Gamma$ and $\Gamma^{*}$. In this case, it is known and actually an immediate consequence of the local representation in Corollary 2.20 that a discrete maximum principle holds true, i.e., a discrete harmonic function can attain its maximum only at the boundary of a closed region. This is not true for general quad-graphs, see for example Skopenkov's paper [23].

Corollary 2.21 Let $f: V\left(\Lambda_{0}\right) \rightarrow \mathbb{C}$.

(i) If $f$ is discrete harmonic, then $\partial_{\Lambda} f$ is discrete holomorphic.

(ii) If $f$ is discrete holomorphic, then $f, \operatorname{Re} f$, and $\operatorname{Im} f$ are discrete harmonic.

Proof By Corollary 2.20, $\triangle f \equiv 4 \bar{\partial}_{\diamond} \partial_{\Lambda} f \equiv 4 \partial_{\diamond} \bar{\partial}_{\Lambda} f$. In particular, $\bar{\partial}_{\diamond} \partial_{\Lambda} f \equiv 0$ if $\triangle f \equiv 0$, which shows (i). Also, $f$ is discrete harmonic if it is discrete holomorphic. Using $\operatorname{Re}(\triangle f) \equiv \triangle \operatorname{Re}(f)$ and $\operatorname{Im}(\triangle f) \equiv \triangle \operatorname{Im}(f), \operatorname{Re}(f)$ and $\operatorname{Im}(f)$ are discrete harmonic if $f$ is.

Similar to Proposition 2.1 that compares the discrete derivative $\partial_{\Lambda}$ with the smooth derivative, the discrete Laplacian coincides with the smooth one up to order one in the general case and up to order two for parallelogram-graphs. This was already shown by Skopenkov in [23]. Since this result follows immediately from our previous ones, we give a proof here as well.

Proposition 2.22 Let $f_{\mathbb{C}}: \mathbb{C} \rightarrow \mathbb{C}$ and $f$ its restriction to $V(\Lambda)$.

(i) If $f_{\mathbb{C}}(z)$ is a polynomial in $\operatorname{Re}(z)$ and $\operatorname{Im}(z)$ of degree at most one, then the smooth and the discrete Laplacian coincide on vertices: $\Delta_{\mathbb{C}} f_{\mathbb{C}}(v)=\Delta f(v)=0$.

(ii) Let all faces of $\Lambda$ be parallelograms. If $f_{\mathbb{C}}(z)$ is a polynomial in $\operatorname{Re}(z)$ and $\operatorname{Im}(z)$ of degree at most two, then the smooth and the discrete Laplacian coincide on vertices: $\triangle_{\mathbb{C}} f_{\mathbb{C}}(v)=\triangle f(v)$. 
Proof (i) Proposition 2.1(ii) says that the function $f(v)=v$ is discrete holomorphic and Corollary 2.21(ii) that real and imaginary part of discrete holomorphic functions are discrete harmonic. Since constants are discrete harmonic, the statement follows.

(ii) In the parallelogram case, let $\hat{Q}$ denote the center of the parallelogram $Q \in F(\Lambda) \cong V(\diamond)$. Analogously to (i), $f(v)=v^{2}$ is discrete harmonic by Proposition 2.1(iii) and Corollary 2.21(ii). Looking at real and imaginary part separately, $\triangle f_{1}^{2} \equiv \Delta f_{2}^{2}$ and $\triangle\left(f_{1} f_{2}\right) \equiv 0$ where we consider $f_{1}(v)=\operatorname{Re}(v), f_{2}(v)=\operatorname{Im}(v)$. Finally,

$$
\triangle|f|^{2} \equiv 4 \partial_{\diamond} \bar{\partial}_{\Lambda}|f|^{2} \equiv 4 \partial_{\diamond} h=4
$$

with $h(Q)=\hat{Q}$ for all $Q \in V(\diamond)$, due to Propositions 2.1(iv) and 2.5 that implied $\bar{\partial}_{\Lambda}|f|^{2} \equiv h$ and $\partial_{\diamond} h \equiv 1$. Since any polynomial in $\operatorname{Re}(z)$ and $\operatorname{Im}(z)$ of monomials of degree two is a linear combination of $f_{1}^{2}-f_{2}^{2}, f_{1}^{2}+f_{2}^{2}$, and $f_{1} f_{2}$, and since we have shown that the discrete Laplacian $\triangle$ and the smooth Laplacian $\triangle_{\mathbb{C}}$ coincide on these, we are done.

Remark The second part of the last proposition generalizes the known result for rhombi given by Chelkak and Smirnov [6]. Note that this is not true for general quadrilaterals even if one assumes that the diagonals of quadrilaterals are orthogonal to each other. For this, consider the following (finite) bipartite quad-graph of Fig. 4: the black vertex 0 is adjacent to the white vertices \pm 1 and $\pm i$ in the quad-graph and adjacent to the black vertices $2+2 i,-1 \pm i$, and $1-i$ in the graph on black vertices. There are no further vertices. Then, $\triangle f(0) \neq 0$ for $f(v)=v^{2}$. Indeed, we would get $\triangle f(0)=0$ if we had replaced $v=2+2 i$ by $v=1+i$ obtaining a rhombic quad-graph; but $\left(\left|\rho_{Q}\right|^{2} / \operatorname{Re}\left(\rho_{Q}\right)\right)(f(v)-f(0))$ scales by a factor of 2 , whereas the other nonzero summands in the formula for $\triangle f(0)$ remain invariant.

In the case of general quad-graphs, smooth functions $f_{\mathbb{C}}: \mathbb{C} \rightarrow \mathbb{C}$, and restrictions $f$ to $V(\Lambda)$, Skopenkov compared the integral of $\Delta_{\mathbb{C}} f_{\mathbb{C}}$ over a square domain $R$ and a sum of $\triangle f(v)$ over black vertices $v$ in $R$ [23]. Moreover, he showed that for $f(v)=|v|^{2}$,

$$
\triangle f(v)=\frac{2}{\operatorname{ar}\left(F_{v}\right)} \sum_{Q_{s} \sim v} \operatorname{area}\left(v v_{s-1}^{\prime} \hat{Q}_{s} v_{s}^{\prime}\right)
$$

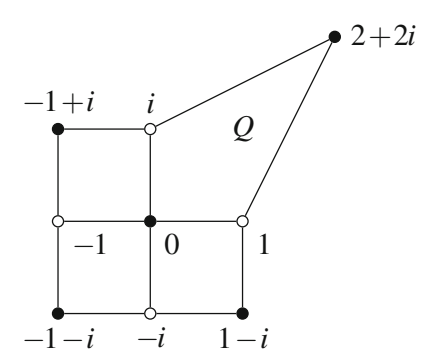

Fig. $4 \Delta f(0) \neq 0$ for $f(v)=v^{2}$ 
when $\hat{Q}_{s}$ is the intersection point of the middle perpendiculars to the diagonals of the quadrilateral $Q_{s}$ (which equals the intersection point of the diagonals if $Q_{s}$ is a parallelogram). Note that in general, $h(Q)=\hat{Q}$ is not discrete holomorphic if $\hat{Q}$ is the intersection of these middle perpendiculars.

Definition For a finite connected subset $\nabla_{0} \subset \diamond$, let $F_{0}$ be the set of faces of $X_{0}$ that correspond to a quadrilateral $Q \in V\left(\diamond_{0}\right)$ that is incident to a vertex in $V\left(\Lambda_{0}\right) \backslash V\left(\partial \Lambda_{0}\right)$. Now, let $f_{1}: V\left(\Lambda_{0}\right) \rightarrow \mathbb{C}$ and $f_{2}: V\left(\Lambda_{0}\right) \backslash V\left(\partial \Lambda_{0}\right) \rightarrow \mathbb{C}$ (or vice versa) be given. Then, we denote by

$$
\left\langle f_{1}, f_{2}\right\rangle:=-\frac{1}{2 i} \iint_{F_{0}} f_{1} \bar{f}_{2} \Omega_{\Lambda}
$$

the discrete scalar product of $f_{1}$ and $f_{2}$ seen as functions on $V\left(\Lambda_{0}\right) \backslash V\left(\partial \Lambda_{0}\right)$.

In the rhombic setup, discrete versions of Green's second identity were already stated by Mercat [19], whose integrals were not well-defined separately, and Chelkak and Smirnov [6], whose boundary integral was an explicit sum involving boundary angles. Skopenkov formulated a discrete Green's second identity with a vanishing boundary term [23].

Theorem 2.23 Let $\nabla_{0} \subset \diamond$ be finite, and let $f, g: V\left(\Lambda_{0}\right) \rightarrow \mathbb{C}$.

(i) Discrete Green's first identity: $\langle f, \triangle g\rangle+\langle d f, d g\rangle=\oint_{\partial X_{0}} f \star d \bar{g}$.

(ii) Discrete Green's second identity: $\langle f, \Delta g\rangle-\langle\Delta f, g\rangle=\oint_{\partial X_{0}}(f \star d \bar{g}-\bar{g} \star d f)$.

Proof (i) Since the discrete exterior derivative is a derivation for the discrete wedge product by Theorem 2.16 ,

$$
d(f \star d \bar{g})=d f \wedge \star d \bar{g}+f \star(\star d \star d \bar{g})=d f \wedge \star d \bar{g}+f \star \Delta \bar{g} .
$$

Now, integration over $F\left(X_{0}\right)$ yields the desired result together with discrete Stokes' Theorem 2.9 and the basic properties of the discrete Hodge star given in Corollary 2.17(ii) and (iv).

(ii) Just apply twice discrete Green's first identity, once with the roles of $f$ and $g$ interchanged, and subtract the equations from another.

The following discrete Weyl's lemma is a direct consequence of discrete Green's second identity, Theorem 2.23(ii). A version for rhombic quad-graphs was given by Mercat in [19], proven by an explicit calculation.

Corollary 2.24 $f: V(\Lambda) \rightarrow \mathbb{C}$ is discrete harmonic if and only if $\langle f, \Delta g\rangle=0$ for every compactly supported $g: V(\Lambda) \rightarrow \mathbb{C}$.

Skopenkov introduced the notion of discrete harmonic conjugates in [23]. We recover his definitions in our notation, observing that his discrete gradient corresponds to the discrete exterior derivative and his counterclockwise rotation by $\pi / 2$ to the discrete Hodge star. 
Definition Let $f$ be a real (discrete harmonic) function on $V\left(\Lambda_{0}\right)$. A real discrete harmonic function $\tilde{f}$ on $V\left(\Lambda_{0}\right)$ is said to be a discrete harmonic conjugate of $f$ if $f+i \tilde{f}$ is discrete holomorphic at all vertices of $\nabla_{0}$.

Note that the existence of a real function $\tilde{f}$ such that $f+i \tilde{f}$ is discrete holomorphic requires already that $f$ is discrete harmonic at all interior vertices of $\Lambda_{0}$ (i.e., $\left.V\left(\Lambda_{0}\right) \backslash V\left(\partial \Lambda_{0}\right)\right)$ due to Corollary 2.21(ii) implying that the real part of a discrete holomorphic function is discrete harmonic.

Lemma 2.25 Let $f: V\left(\Lambda_{0}\right) \rightarrow \mathbb{R}$ satisfy $\triangle f(v)=0$ for all $v \in V\left(\Lambda_{0}\right) \backslash V\left(\partial \Lambda_{0}\right)$.

(i) The discrete harmonic conjugate $\tilde{f}$ is unique up to two additive real constants on $\Gamma_{0}$ and $\Gamma_{0}^{*}$.

(ii) If $\diamond_{0}$ forms a simply-connected closed region, then a discrete harmonic conjugate $\tilde{f}$ exists.

Proof (i) If $\tilde{f}_{1}$ and $\tilde{f}_{2}$ are two real discrete harmonic conjugates, then their difference $\tilde{f}_{1}-\tilde{f}_{2}$ is real and discrete holomorphic at all vertices of $\nabla_{0}$. So by Proposition 2.2(ii) $\left(\diamond_{0}\right.$ is connected), it is biconstant as a real discrete holomorphic function.

(ii) Since $f$ is harmonic, $d \star d f=0$, i.e., $\star d f$ is closed and of type $\nabla$. Moreover, reality of $f$ implies $\star d f=-i \partial_{\Lambda} f d z+i \bar{\partial}_{\Lambda} f d \bar{z}=2 \operatorname{Im}\left(\partial_{\Lambda} f d z\right)$. So in the same manner as in the proof of Proposition 2.8 (existence of a discrete primitive if $\nabla_{0}$ forms a simply-connected closed region), $\star d f$ can be integrated to a real function $\tilde{f}$ on $V\left(\Lambda_{0}\right)$. Since

$$
d(f+i \tilde{f})=d f+i \star d f=2 \operatorname{Re}\left(\partial_{\Lambda} f d z\right)+2 i \operatorname{Im}\left(\partial_{\Lambda} f d z\right)=2 \partial_{\Lambda} f d z
$$

is of the form $p d z$ and of type $\nabla, f+i \tilde{f}$ is discrete holomorphic by Corollary 2.12.

Note that in the case of quadrilaterals with orthogonal diagonals, such that $\triangle$ splits into two discrete Laplacians on $\Gamma$ and $\Gamma^{*}$, it follows that a discrete harmonic conjugate of a discrete harmonic function on $V(\Gamma)$ can be defined on $V\left(\Gamma^{*}\right)$ and vice versa, as was already noted by Chelkak and Smirnov in [6].

Corollary 2.26 Let $f: V\left(\Lambda_{0}\right) \rightarrow \mathbb{C}$ be discrete holomorphic at all vertices of $\searrow_{0}$. Then, $\operatorname{Im}(f)$ is uniquely determined by $\operatorname{Re}(f)$ up to two additive constants on $\Gamma_{0}$ and $\Gamma_{0}^{*}$.

\subsubsection{Discrete Dirichlet Energy}

We follow the classical approach of discretizing the Dirichlet energy introduced by Mercat in [21]. Note that Skopenkov's definition in [23] is exactly the same. In particular, Skopenkov's results, including an approximation property of the Laplacian, convergence of the discrete Dirichlet energy to the smooth Dirichlet energy for nondegenerate uniform sequences of quad-graphs, and further theorems for quad-graphs with orthogonal diagonals apply as well in our setting. We refer to his work [23] for details on these results. 
Definition For a function $f: V\left(\Lambda_{0}\right) \rightarrow \mathbb{C}$, we define the discrete Dirichlet energy of $f$ on $\diamond_{0}$ as $E_{\diamond_{0}}(f):=\langle d f, d f\rangle \in[0, \infty]$.

If $\nabla_{0}$ is finite, then the discrete Dirichlet boundary value problem asks for a real function $f$ on $V\left(\Lambda_{0}\right)$ such that $f$ is discrete harmonic at all points of $V\left(\Lambda_{0}\right) \backslash V\left(\partial \Lambda_{0}\right)$ and such that $f$ agrees with a preassigned real function $f_{0}$ on the boundary $V\left(\partial \Lambda_{0}\right)$.

Proposition 2.27 Let $\nabla_{0} \subseteq \diamond$ be finite, and let $f: V\left(\Lambda_{0}\right) \rightarrow \mathbb{C}$. Then,

$$
\begin{aligned}
E_{\diamond_{0}}(f) & =\sum_{Q \in V\left(\diamond_{0}\right)} \frac{1}{2 \operatorname{Re}\left(\rho_{Q}\right)}\left(\left|\rho_{Q}\right|^{2}\left|f\left(b_{+}\right)-f\left(b_{-}\right)\right|^{2}+\left|f\left(w_{+}\right)-f\left(w_{-}\right)\right|^{2}\right) \\
& +\sum_{Q \in V\left(\diamond_{0}\right)} \frac{\operatorname{Im}\left(\rho_{Q}\right)}{\operatorname{Re}\left(\rho_{Q}\right)} \operatorname{Re}\left(\left(f\left(b_{+}\right)-f\left(b_{-}\right)\right) \overline{\left(f\left(w_{+}\right)-f\left(w_{-}\right)\right)}\right) .
\end{aligned}
$$

Proof Since $E_{\diamond_{0}}(f)$ is a sum over $Q \in V\left(\diamond_{0}\right)$, it suffices to check the identity for just a singular quadrilateral $Q$. Furthermore, $E_{\diamond_{0}}(f)=E_{\diamond_{0}}(\operatorname{Re}(f))+E_{\diamond_{0}}(\operatorname{Im}(f))$ allows us to restrict to real functions $f$. Then, $E_{Q}(f)$ equals

$$
\begin{aligned}
\iint_{F_{Q}} d f \wedge \star d f & =4 \operatorname{area}(Q) \partial_{\Lambda} f(Q) \bar{\partial}_{\Lambda} f(Q) \\
& =2\left|w_{+}-w_{-}\right|\left|b_{+}-b_{-}\right| \sin \left(\varphi_{Q}\right)\left|\bar{\partial}_{\Lambda} f(Q)\right|^{2} .
\end{aligned}
$$

Here, $b_{-}, w_{-}, b_{+}, w_{+}$are the vertices of $Q$ in counterclockwise order, starting with a black vertex, and $F_{Q}$ is the face of $X$ corresponding to $Q$.

In the proof of Proposition 2.1(i), we calculated

$$
\bar{\partial}_{\Lambda} f(Q)=\frac{\left(w_{+}-w_{-}\right)\left(f\left(b_{+}\right)-f\left(b_{-}\right)\right)-\left(b_{+}-b_{-}\right)\left(f\left(w_{+}\right)-f\left(w_{-}\right)\right)}{2 i\left|w_{+}-w_{-}\right|\left|b_{+}-b_{-}\right| \sin \left(\varphi_{Q}\right)} .
$$

It follows that $E_{Q}(f)$ equals

$$
\begin{aligned}
& \frac{\left|w_{+}-w_{-}\right|}{2\left|b_{+}-b_{-}\right| \sin \left(\varphi_{Q}\right)}\left|f\left(b_{+}\right)-f\left(b_{-}\right)\right|^{2}+\frac{\left|b_{+}-b_{-}\right|}{2\left|w_{+}-w_{-}\right| \sin \left(\varphi_{Q}\right)}\left|f\left(w_{+}\right)-f\left(w_{-}\right)\right|^{2} \\
& -\operatorname{Re}\left(\frac{\left(w_{+}-w_{-}\right) \overline{\left(b_{+}-b_{-}\right)}}{\left|w_{+}-w_{-}\right|\left|b_{+}-b_{-}\right| \sin \left(\varphi_{Q}\right)}\left(f\left(b_{+}\right)-f\left(b_{-}\right)\right)\left(f\left(w_{+}\right)-f\left(w_{-}\right)\right)\right) .
\end{aligned}
$$

Remembering $\rho_{Q}=-i \exp \left(i \varphi_{Q}\right)\left|w_{+}-w_{-}\right| /\left|b_{+}-b_{-}\right|$, the claim follows from

$$
\begin{aligned}
\frac{\left|w_{+}-w_{-}\right|}{2\left|b_{+}-b_{-}\right| \sin \left(\varphi_{Q}\right)} & =\frac{\left|\rho_{Q}\right|^{2}}{2 \operatorname{Re}\left(\rho_{Q}\right)}, \quad \frac{\left|b_{+}-b_{-}\right|}{2\left|w_{+}-w_{-}\right| \sin \left(\varphi_{Q}\right)}=\frac{1}{2 \operatorname{Re}\left(\rho_{Q}\right)}, \\
- & \operatorname{Re}\left(\frac{\left(w_{+}-w_{-}\right) \overline{\left(b_{+}-b_{-}\right)}}{\left|w_{+}-w_{-}\right|\left|b_{+}-b_{-}\right| \sin \left(\varphi_{Q}\right)}\right)=\frac{\operatorname{Im}\left(\rho_{Q}\right)}{\operatorname{Re}\left(\rho_{Q}\right)}
\end{aligned}
$$


The same formula of $E_{\diamond_{0}}(f)$ was given by Mercat [21].

In the case of rhombic quad-graphs, Duffin proved in [10] that the discrete Dirichlet boundary value problem has a unique solution. The same argument applies for general quad-graphs with the discrete Dirichlet energy defined here. Using a different notation, Skopenkov proved existence and uniqueness of solutions of the discrete Dirichlet boundary value problem as well [23].

Lemma 2.28 Let $\nabla_{0} \subset \diamond$ be finite and $f_{0}: V\left(\partial \Lambda_{0}\right) \rightarrow \mathbb{R}$. We consider the affine space of real functions $f: V\left(\Lambda_{0}\right) \rightarrow \mathbb{R}$ that agree with $f_{0}$ on the boundary.

Then, $E_{\diamond_{0}}$ is a strictly convex nonnegative quadratic functional in terms of the interior values $f(v)$. Furthermore,

$$
-\frac{\partial E_{\diamond_{0}}}{\partial f(v)}(f)=2 \operatorname{ar}\left(F_{v}\right) \triangle f(v)
$$

for any $v \in V\left(\Lambda_{0}\right) \backslash V\left(\partial \Lambda_{0}\right)$. In particular, the solution of the discrete Dirichlet boundary value problem is given by the unique minimizer of $E_{\diamond_{0}}$.

Proof By construction, $E_{\diamond_{0}}$ is a quadratic form in the vector space of real functions $f: V\left(\Lambda_{0}\right) \backslash V\left(\partial \Lambda_{0}\right) \rightarrow \mathbb{R}$. In particular, it is convex, nonnegative, and quadratic in terms of the values $f(v)$. Thus, global minima exist. To prove strict convexity, it suffices to check that the minimum is unique.

For an interior vertex $v_{0} \in V\left(\Lambda_{0}\right) \backslash V\left(\partial \Lambda_{0}\right)$, let $\phi(v):=\delta_{v v_{0}}$ be the Kronecker delta function on $V\left(\Lambda_{0}\right)$. Then,

$$
\frac{\partial E_{\diamond_{0}}}{\partial f\left(v_{0}\right)}(f)=\left.\frac{d}{d t} E_{\diamond_{0}}(f+t \phi)\right|_{t=0}=2\langle d f, d \phi\rangle=-2\langle\Delta f, \phi\rangle=-2 \operatorname{ar}\left(F_{v_{0}}\right) \triangle f\left(v_{0}\right)
$$

due to Proposition 2.19 that stated that $\delta$ is the formal adjoint of $d$. To apply the proposition, we consider $\phi$ as a function on $V(\Lambda)$ and extend $f$ to $V(\Lambda)$ by setting it zero on $V(\Lambda) \backslash V\left(\Lambda_{0}\right)$. This changes neither $\langle d f, d \phi\rangle$ nor $2\langle\triangle f, \phi\rangle$.

It follows that exactly the minima of $E_{\diamond_{0}}$ are discrete harmonic and therefore solve the discrete Dirichlet boundary value problem. The difference $g$ of two minima is a discrete harmonic function vanishing on the boundary. Similar to the argument given in the previous paragraph, $E_{\diamond_{0}}(g)=\langle d g, d g\rangle=-\langle\triangle g, g\rangle=0$ by Proposition 2.19 since $g$ is zero on $V\left(\partial \Lambda_{0}\right)$. But only biconstant functions have zero energy. Thus, the difference has to vanish everywhere, i.e., minima are unique.

In the following, we apply Lemma 2.28 to show that $\partial_{\Lambda}, \bar{\partial}_{\Lambda}, \partial_{\diamond}, \bar{\partial} \diamond, \triangle$ are surjective operators. This implies immediately the existence of discrete Green's functions and discrete Cauchy's kernels, as we will see in Sects. 2.5 and 2.6.

Lemma 2.29 Let $\nabla_{0} \subset \diamond$ be finite and assume that it forms a simply-connected closed region. Then, the discrete derivatives $\partial_{\Lambda}, \bar{\partial}_{\Lambda}, \partial_{\diamond}, \bar{\partial}_{\diamond}$ and the discrete Laplacian $\triangle$ are surjective operators. That means, given any complex functions $h_{0}$ on $V\left(\diamond_{0}\right)$ and $f_{0}$ on $V\left(\Lambda_{0}\right) \backslash V\left(\partial \Lambda_{0}\right)$, there exist functions $h_{\partial}, h_{\bar{\partial}}$ on $V\left(\diamond_{0}\right)$ and 
$f_{\partial}, f_{\bar{\partial}}, f_{\triangle}$ on $V\left(\Lambda_{0}\right)$ such that $\partial_{\diamond} h_{\partial}=\bar{\partial}_{\diamond} h_{\bar{\partial}}=\Delta f_{\triangle}=f_{0}$ and $\partial_{\Lambda} f_{\partial}=\bar{\partial}_{\Lambda} f_{\bar{\partial}}=h_{0}$. If $f_{0}$ is real-valued, then $f_{\triangle}$ can be chosen real-valued as well.

Proof Denote by $B$ the number of vertices of $\partial \Lambda_{0}$. By assumption, $\partial \Lambda_{0}$ is a simple closed broken line with $B$ edges.

By the previous Lemma 2.28, the space of real discrete harmonic functions on $V\left(\Lambda_{0}\right)$ has dimension $B$. Clearly, real and imaginary part of a discrete harmonic function are itself discrete harmonic. Therefore, the complex dimension of the space of complex discrete harmonic functions, i.e., of the kernel of $\triangle$, is $B$ as well. Thus, $\triangle: \mathbb{K}^{V\left(\Lambda_{0}\right)} \rightarrow \mathbb{K}^{V\left(\Lambda_{0} \backslash \partial \Lambda_{0}\right)}$ is a surjective linear operator with $\mathbb{K} \in\{\mathbb{R}, \mathbb{C}\}$.

Now, $\triangle=4 \partial_{\diamond} \bar{\partial}_{\Lambda}=4 \bar{\partial}_{\diamond} \partial_{\Lambda}$ by Corollary 2.20 , so $\partial_{\diamond}, \bar{\partial}_{\diamond}: \mathbb{C}^{V\left(\diamond_{0}\right)} \rightarrow \mathbb{C}^{V\left(\Lambda_{0} \backslash \partial \Lambda_{0}\right)}$ are surjective as well. The kernel of $\bar{\partial}_{\diamond}$ consists of all discrete holomorphic functions on $V\left(\diamond_{0}\right)$. By Proposition 2.8 ( $\diamond_{0}$ forms a simply-connected closed region), any such function has a discrete primitive, i.e., the kernel is contained in the image of $\partial_{\Lambda}$. Using the surjectivity of $\triangle$, it follows that $\partial_{\Lambda}: \mathbb{C}^{V\left(\Lambda_{0}\right)} \rightarrow \mathbb{C}^{V\left(\diamond_{0}\right)}$ is surjective. The same is true for $\bar{\partial}_{\Lambda}$.

Theorem 2.30 The discrete derivatives $\partial_{\Lambda}, \bar{\partial}_{\Lambda}, \partial_{\diamond}, \bar{\partial}_{\diamond}$ and the discrete Laplacian $\triangle$ (defined on complex or real functions) are surjective operators on the vector space of functions on $V(\Lambda)$ or $V(\diamond)$.

Proof Let $\nabla_{0} \subset \diamond_{1} \subset \diamond_{2} \subset \ldots \subset \diamond$ be a sequence of finite subgraphs forming simply-connected closed regions such that $\bigcup_{k=0}^{\infty} \diamond_{k}=\diamond$. By $\Lambda_{k}$ we denote the subgraph of $\Lambda$ whose vertices and edges are the vertices and edges of quadrilaterals in $\nabla_{k}$.

Let us first prove that any $h: V(\diamond) \rightarrow \mathbb{C}$ has a preimage under the discrete derivatives $\partial_{\Lambda}, \bar{\partial}_{\Lambda}$. By the previous Lemma 2.29, the affine space $A_{k}^{(0)}$ of all complex functions on $V\left(\Lambda_{k}\right)$ that are mapped to $\left.h\right|_{V\left(\diamond_{k}\right)}$ by $\partial_{\Lambda}$ (or $\bar{\partial}_{\Lambda}$ ) is nonempty. Let $\left.A_{k}^{(0)}\right|_{\Lambda_{j}}$ denote the affine space of restrictions of these functions to $V\left(\Lambda_{j}\right) \subseteq V\left(\Lambda_{k}\right)$. Clearly,

$$
\left.\left.A_{0}^{(0)} \supseteq A_{1}^{(0)}\right|_{\Lambda_{0}} \supseteq A_{2}^{(0)}\right|_{\Lambda_{0}} \supseteq \cdots
$$

Since all affine spaces are finite-dimensional and nonempty, this chain becomes stationary at some point, giving a function $f_{0}$ on $V\left(\Lambda_{0}\right)$ mapped to $\left.h\right|_{V\left(\vartheta_{0}\right)}$ by $\partial_{\Lambda}$ (or $\left.\bar{\partial}_{\Lambda}\right)$ that can be extended to a function in $A_{k}^{(0)}$ for any $k$.

Inductively, assume that $f_{j}: V\left(\Lambda_{j}\right) \rightarrow \mathbb{C}$ is mapped to $\left.h\right|_{V\left(\diamond_{j}\right)}$ by $\partial_{\Lambda}\left(\right.$ or $\left.\bar{\partial}_{\Lambda}\right)$ and that $f_{j}$ can be extended to a function in $A_{k}^{(j)}$ for all $k \geqslant j$. Let $A_{k}^{(j+1)}, k \geqslant j+1$, be the affine space of all complex functions on $V\left(\Lambda_{k}\right)$ that are mapped to $\left.h\right|_{V\left(\diamond_{k}\right)}$ by $\partial_{\Lambda}$ (or $\left.\bar{\partial}_{\Lambda}\right)$ and whose restriction to $V\left(\Lambda_{j}\right)$ is equal to $f_{j}$. By assumption, all these spaces are nonempty. In the same way as above, there is a function $f_{j+1}$ extending $f_{j}$ to $V\left(\Lambda_{j+1}\right)$ that is mapped to $\left.h\right|_{V\left(\diamond_{j+1}\right)}$ by $\partial_{\Lambda}$ (or $\bar{\partial}_{\Lambda}$ ) and that can be extended to a function in $A_{k}^{(j+1)}$ for all $k \geqslant j+1$.

For $v \in V\left(\Lambda_{k}\right)$, define $f(v):=f_{k}(v) . f$ is a well-defined complex function on $V(\Lambda)$ with $\partial_{\Lambda} f=h\left(\right.$ or $\left.\bar{\partial}_{\Lambda} f=h\right)$. Hence, $\partial_{\Lambda}, \bar{\partial}_{\Lambda}: \mathbb{C}^{V(\Lambda)} \rightarrow \mathbb{C}^{V(\diamond)}$ are surjective. 
Replacing $V\left(\diamond_{k}\right)$ by $V\left(\Lambda_{k}\right) \backslash V\left(\partial \Lambda_{k}\right)$, we obtain with the same arguments that $\triangle$ is surjective, regardless whether $\Delta$ is defined on real or complex functions. Finally, $\partial_{\diamond}, \bar{\partial}_{\diamond}: \mathbb{C}^{V(\diamond)} \rightarrow \mathbb{C}^{V(\Lambda)}$ are surjective due to $\Delta=4 \partial_{\diamond} \bar{\partial}_{\Lambda}=4 \bar{\partial}_{\diamond} \partial_{\Lambda}$ by Corollary 2.20 .

In the case of rhombic quad-graphs with bounded interior angles, Kenyon proved the existence of a discrete Green's function and a discrete Cauchy's kernel with asymptotic behaviors similar to the classical setting [16]. But in the general case, it seems to be practically impossible to speak about any asymptotic behavior of certain discrete functions. For this reason, we will consider functions that discretize Green's functions and Cauchy's kernels apart from their asymptotics in Sects. 2.5 and 2.6. Not requiring a certain asymptotic behavior leads to non-uniqueness of these functions.

Still, one can expect results concerning the asymptotics of special discrete functions if the interior angles and the side lengths of the quadrilaterals are bounded, meaning that the quadrilaterals do not degenerate at infinity. And indeed, on such quad-graphs any discrete harmonic function whose difference functions on $V(\Gamma)$ and $V\left(\Gamma^{*}\right)$ have asymptotics $o\left(v^{-1 / 2}\right)$ as $|v| \rightarrow \infty$ is biconstant. In the rhombic setting, Chelkak and Smirnov showed that a discrete Liouville's theorem holds true, i.e., any bounded discrete harmonic function on $V(\Lambda)$ vanishes [6].

Theorem 2.31 Assume that there exist constants $\alpha_{0}>0$ and $E_{1} \geqslant E_{0}>0$ such that $\alpha \geqslant \alpha_{0}$ and $E_{1} \geqslant e \geqslant E_{0}$ for all interior angles $\alpha$ and side lengths ef quadrilaterals $Q \in F(\Lambda)$. If $f: V(\Lambda) \rightarrow \mathbb{C}$ is discrete harmonic and $f\left(v_{+}\right)-f\left(v_{-}\right)=o\left(v_{ \pm}^{-1 / 2}\right)$ for any two adjacent $v_{ \pm} \in V(\Gamma)$ or $v_{ \pm} \in V\left(\Gamma^{*}\right)$ as $\left|v_{ \pm}\right| \rightarrow \infty$, then $f$ is biconstant.

Proof Without loss of generality, we can restrict to real functions $f$. Assume that $f$ is not biconstant. Then, $d f \wedge \star d f$ is nonzero somewhere on a face $F$ of $X$. In particular, the discrete Dirichlet energy of $f$ is bounded away from zero if a domain contains $F$. Now, the idea of proof is to show that if the domain is large enough but still compact, the function being zero in the interior and equal to $f$ on the boundary has a smaller discrete Dirichlet energy than $f$, contradicting Lemma 2.28 that implies that $f$ is the unique minimizer of the discrete Dirichlet energy on that domain.

Let us first bound the intersection angles and the lengths of diagonals of the quadrilaterals. Take $Q \in F(\Lambda)$ and denote its vertices by $b_{-}, w_{-}, b_{+}, w_{+}$in counterclockwise order, starting with a black vertex. Then, there are two opposite interior angles that are less than $\pi$, say $\alpha_{ \pm}$at vertices $b_{ \pm}$. Since all interior angles are bounded by $\alpha_{0}$ from below, one of $\alpha_{ \pm}$is less than or equal to $\pi-\alpha_{0}$, say $\alpha_{0} \leqslant \alpha_{-} \leqslant \pi-\alpha_{0}$.

By triangle inequality, $\left|b_{+}-b_{-}\right|,\left|w_{+}-w_{-}\right|<2 E_{1}$. Twice the area of $Q$ equals

$$
\left|w_{-}-b_{-}\right|\left|w_{+}-b_{-}\right| \sin \left(\alpha_{-}\right)+\left|w_{-}-b_{+}\right|\left|w_{+}-b_{+}\right| \sin \left(\alpha_{+}\right) \geqslant E_{0}^{2} \sin \left(\alpha_{0}\right),
$$

so $\left|b_{+}-b_{-}\right|\left|w_{+}-w_{-}\right| \sin \left(\varphi_{Q}\right)=2 \operatorname{area}(Q) \geqslant E_{0}^{2} \sin \left(\alpha_{0}\right)$. It follows that

$$
\left|b_{+}-b_{-}\right| \geqslant \frac{E_{0}^{2} \sin \left(\alpha_{0}\right)}{\left|w_{+}-w_{-}\right| \sin \left(\varphi_{Q}\right)}>\frac{E_{0}^{2} \sin \left(\alpha_{0}\right)}{2 E_{1}}=: E_{0}^{\prime} .
$$


Similarly, $\left|w_{+}-w_{-}\right|>E_{0}^{\prime}$ and $\sin \left(\varphi_{Q}\right)>E_{0}^{\prime} /\left(2 E_{1}\right)$. Thus, we can bound

$$
\begin{gathered}
\rho_{Q}=\frac{\left|w_{+}-w_{-}\right|}{\left|b_{+}-b_{-}\right|} \exp \left(i\left(\varphi_{Q}-\frac{\pi}{2}\right)\right)=\frac{\left|w_{+}-w_{-}\right|}{\left|b_{+}-b_{-}\right|}\left(\sin \left(\varphi_{Q}\right)-i \cos \left(\varphi_{Q}\right)\right) \\
\text { by }\left|\rho_{Q}\right|<\frac{2 E_{1}}{E_{0}^{\prime}} \text { and } \operatorname{Re}\left(\rho_{Q}\right)>\left(\frac{E_{0}^{\prime}}{2 E_{1}}\right)^{2} .
\end{gathered}
$$

For some $r>0$, denote by $B_{\diamond}(0, r) \subset V(\diamond)$ the set of quadrilaterals that have a nonempty intersection with the open ball $B(0, r)$ around 0 and radius $r$. Let $R>2 E_{1}$, and consider the ball $B_{\diamond}(0, R) \subset V(\diamond)$. Since $\Lambda$ is locally finite, $B_{\diamond}(0, R)$ is finite. Also, if we connect two elements of $B_{\diamond}(0, R)$ if they are adjacent in $\diamond$, then we obtain a connected subgraph of $\diamond$ that we will also denote by $B_{\diamond}(0, R)$. To see that it is connected, we observe that the closed region in the complex plane formed by the quadrilaterals in $B_{\diamond}(0, R)$ is connected, and that if $Q \in B_{\diamond}(0, R)$, then one of its corners, say $v$, has to lie in $B(0, R)$ and so all quadrilaterals incident to $v$ are in $B_{\diamond}(0, R)$. We denote by $\Lambda_{R}$ the subgraph of $\Lambda$ that consists of all the vertices and edges of quadrilaterals in $B_{\diamond}(0, R)$

Since edge lengths are bounded by $E_{1}$, all elements of $B_{\diamond}(0, R)$ that are not completely contained in $B(0, R)$ are contained in $B\left(0, R+2 E_{1}\right) \backslash B\left(0, R-2 E_{1}\right)$. The area of the latter is $8 \pi R E_{1}$. Any quadrilateral has area at least $E_{0}^{2} \sin \left(\alpha_{0}\right) / 2$, so at most $16 \pi R E_{1} /\left(E_{0}^{2} \sin \left(\alpha_{0}\right)\right)$ quadrilaterals of $B_{\diamond}(0, R)$ do not lie completely in $B(0, R)$. We call these quadrilaterals for short boundary faces.

Consider the real function $f_{R}$ defined on $V\left(\Lambda_{R}\right)$ that is equal to $f$ at $V\left(\partial \Lambda_{R}\right)$ and equal to 0 in $V\left(\Lambda_{R}\right) \backslash V\left(\partial \Lambda_{R}\right)$. When computing the discrete Dirichlet energy of $f_{R}$ on $B_{\diamond}(0, R)$, only boundary faces can give nonzero contributions. If we look at the formula of the discrete Dirichlet energy in Proposition 2.27 and use in addition that $f\left(v_{+}\right)-f\left(v_{-}\right)=o\left(R^{-1 / 2}\right)$ for vertices of boundary faces, then we see that any contribution of a boundary face has asymptotics $o\left(R^{-1}\right)$. For this, we use that $\left.\mid \operatorname{Re}\left(\rho_{Q}\right)\right) \mid$ is bounded from below by a constant and $\left|\operatorname{Im}\left(\rho_{Q}\right)\right| \leqslant\left|\rho_{Q}\right|<2 E_{1} / E_{0}^{\prime}$. Using that there are only $O(R)$ faces in the boundary (the constant depending on $E_{0}, E_{1}, \alpha_{0}$ only), the discrete Dirichlet energy $E_{B_{\diamond}(0, R)}\left(f_{R}\right)$, considered as a function of $R$, behaves as $o(1)$. So if $R$ is large enough, then

$$
E_{B_{\diamond}(0, R)}\left(f_{R}\right)<\iint_{F} d f \wedge \star d f \leqslant E_{B_{\diamond}(0, R)}(f),
$$

contradicting that $f$ minimizes the discrete Dirichlet energy by Lemma 2.28.

\subsection{Discrete Green's Functions}

Definition Let $v_{0} \in V(\Lambda)$. A real function $G\left(\cdot ; v_{0}\right)$ on $V(\Lambda)$ is a $($ free $)$ discrete Green's function for $v_{0}$ if 


$$
G\left(v_{0} ; v_{0}\right)=0 \text { and } \triangle G\left(v ; v_{0}\right)=\frac{1}{2 \operatorname{ar}\left(F_{v_{0}}\right)} \delta_{v v_{0}} \text { for all } v \in V(\Lambda) .
$$

Remark It is important to note that discrete Green's functions as we defined them are far from being unique. The contrast to the smooth setting or the rhombic case investigated by Kenyon [16], Chelkak and Smirnov [6] is that no asymptotics are required. So it might be more appropriate to call these functions functions of discrete Green's function type, but for the sake of convenience, we still call them discrete Green's functions.

However, when considering planar parallelogram-graphs with bounded interior angles and bounded ratio of side lengths in Sect.3.3, existence of a discrete Green's function with asymptotics generalizing the corresponding result for rhombic quadgraphs is proven.

As a corollary of Theorem 2.30 we get existence of discrete Green's functions.

Corollary 2.32 A discrete Green's function exists for any $v_{0} \in V(\Lambda)$.

Proof By Theorem 2.30, $\triangle$ is surjective, so there exists a function $G: V(\Lambda) \rightarrow \mathbb{R}$ with $\triangle G(v)=\delta_{v v_{0}} /\left(2 \operatorname{ar}\left(F_{v_{0}}\right)\right)$. Since constant functions are discrete harmonic, we can adjust $G$ to get $G\left(v_{0}\right)=0$.

The following notion of discrete Greens' functions in a discrete domain follows the presentation of Chelkak and Smirnov in [6].

Definition Let $\nabla_{0} \subset \diamond$ be finite and form a simply-connected closed region. For a vertex $v_{0} \in V\left(\Lambda_{0}\right) \backslash V\left(\partial \Lambda_{0}\right)$, a real function $G_{\Lambda_{0}}\left(\cdot ; v_{0}\right)$ on $V\left(\Lambda_{0}\right)$ is a discrete Green's function in $\Lambda_{0}$ for $v_{0}$ if

$$
\begin{aligned}
G_{\Lambda_{0}}\left(v ; v_{0}\right) & =0 \text { for all } v \in V\left(\partial \Lambda_{0}\right) \\
\text { and } \triangle G_{\Lambda_{0}}\left(v ; v_{0}\right) & =\frac{1}{2 \operatorname{ar}\left(F_{v_{0}}\right)} \delta_{v v_{0}} \text { for all } v \in V\left(\Lambda_{0}\right) \backslash V\left(\partial \Lambda_{0}\right) .
\end{aligned}
$$

An immediate corollary of Lemma 2.29 is now the existence of these functions.

Corollary 2.33 Let $\nabla_{0} \subset \diamond$ be finite and form a simply-connected closed region. Furthermore, let $v_{0} \in V\left(\Lambda_{0}\right) \backslash V\left(\partial \Lambda_{0}\right)$. Then, there exists a unique discrete Green's function in $\Lambda_{0}$ for $v_{0}$.

Proof Due to our assumptions on $\diamond_{0}$, existence follows from Lemma 2.29 stating surjectivity of $\Delta$ on such domains. Since the difference of two discrete Green's functions in $\Lambda_{0}$ for $v_{0}$ is discrete harmonic on $V\left(\Lambda_{0}\right)$ and equals zero on the boundary $V\left(\partial \Lambda_{0}\right)$, it has to be identically zero by Lemma 2.28 since the zero function is the unique solution of the corresponding discrete Dirichlet boundary value problem. 


\subsection{Discrete Cauchy's Integral Formulae}

In this section, we first formulate discretizations of the standard Cauchy's integral formula, both for discrete holomorphic functions on $V(\Lambda)$ and $V(\diamond)$. Later, we give with Theorem 2.36 a discrete formulation of Cauchy's integral formula for the derivative of a holomorphic function. We conclude this part with Sect.2.6.1, where we relate our formulation of the discrete Cauchy's integral formula for discrete holomorphic functions on $V(\diamond)$ with Chelkak's and Smirnov's notation in [6].

Definition Discrete Cauchy's kernels with respect to $Q_{0} \in V(\diamond)$ and $v_{0} \in V(\Lambda)$ are functions $K_{Q_{0}}: V(\Lambda) \rightarrow \mathbb{C}$ and $K_{v_{0}}: V(\diamond) \rightarrow \mathbb{C}$, respectively, that satisfy for all $Q \in V(\diamond), v \in V(\Lambda)$ :

$$
\bar{\partial}_{\Lambda} K_{Q_{0}}(Q)=\delta_{Q Q_{0}} \frac{\pi}{\operatorname{ar}\left(F_{Q}\right)} \quad \text { and } \quad \bar{\partial}_{\diamond} K_{v_{0}}(v)=\delta_{v v_{0}} \frac{\pi}{\operatorname{ar}\left(F_{v}\right)} .
$$

For fixed $Q_{0} \in V\left(\diamond_{0}\right), v_{0} \in V\left(\Lambda_{0}\right) \backslash V\left(\partial \Lambda_{0}\right)$, functions $K_{Q_{0}}: V\left(\Lambda_{0}\right) \rightarrow \mathbb{C}$ and $K_{v_{0}}: V\left(\oslash_{0}\right) \rightarrow \mathbb{C}$ satisfying the above equations for all faces $Q \in V\left(\searrow_{0}\right)$ and vertices $v \in V\left(\Lambda_{0}\right) \backslash V\left(\partial \Lambda_{0}\right)$ are called discrete Cauchy's kernels on $V\left(\Lambda_{0}\right)$ or $V\left(\diamond_{0}\right)$, respectively.

Clearly, the restrictions of discrete Cauchy's kernels to $V\left(\Lambda_{0}\right)$ or $V\left(\diamond_{0}\right)$, respectively, are discrete Cauchy's kernels on $V\left(\Lambda_{0}\right)$ or $V\left(\diamond_{0}\right)$, respectively.

Remark It is important to note that exactly as discrete Green's functions, discrete Cauchy's kernels as we defined them are far from being unique. Again, it might be more appropriate to call these functions functions of discrete Cauchy's kernel type, but we still call them discrete Cauchy's kernels.

But if interior angles and side lengths of quadrilaterals are bounded, then it follows from Theorem 2.31 that any discrete Cauchy's kernel with respect to a vertex of $\diamond$ with asymptotics $o\left(v^{-1 / 2}\right)$ as $|v| \rightarrow \infty$ is necessarily unique, but we cannot prove existence so far. In Sect. 3.4, explicit formulae for discrete Cauchy's kernels with asymptotics similar to the smooth setting are given, generalizing Kenyon's result [16] on rhombic quad-graphs to parallelogram-graphs.

The existence of discrete Cauchy's kernels follows from the surjectivity of discrete derivatives by Theorem 2.30:

Corollary 2.34 Let $Q_{0} \in V(\diamond)$ and $v_{0} \in V(\Lambda)$ be arbitrary. Then, discrete Cauchy's kernels with respect to $Q_{0}$ and $v_{0}$ exist.

Theorem 2.35 Let $f$ and $h$ be discrete holomorphic functions on $V\left(\Lambda_{0}\right)$ and $V\left(\searrow_{0}\right)$, respectively. Furthermore, let $v_{0} \in V\left(\Lambda_{0}\right) \backslash V\left(\partial \Lambda_{0}\right)$ and $Q_{0} \in V\left(\nabla_{0}\right)$ be given, and let $K_{v_{0}}: V(\diamond) \rightarrow \mathbb{C}$ and $K_{Q_{0}}: V(\Lambda) \rightarrow \mathbb{C}$ be discrete Cauchy's kernels with respect to $v_{0}$ and $Q_{0}$ on $V\left(\nabla_{0}\right)$ and $V\left(\Lambda_{0}\right)$, respectively.

Then, for any discrete contours $C_{v_{0}}$ and $C_{Q_{0}}$ on $X_{0}$ surrounding $v_{0}$ and $Q_{0}$, respectively, once in counterclockwise order, discrete Cauchy's integral formulae hold: 


$$
f\left(v_{0}\right)=\frac{1}{2 \pi i} \oint_{C_{v_{0}}} f K_{v_{0}} d z \text { and } h\left(Q_{0}\right)=\frac{1}{2 \pi i} \oint_{C_{Q_{0}}} h K_{Q_{0}} d z .
$$

Proof Let $P_{v}$ and $P_{Q}$ be discrete elementary cycles, $v$ being an interior vertex and $Q$ an interior face of $\Lambda_{0}$. By Lemma 2.3 that relates $\partial_{\Lambda}, \bar{\partial}_{\Lambda}$ with discrete contour integrals and the definition of $\bar{\partial}_{\diamond}$, we get:

$$
\begin{aligned}
& \frac{1}{2 \pi i} \oint_{P_{v}} f K_{v_{0}} d z=\frac{1}{\pi} \operatorname{ar}\left(F_{v}\right) f(v) \bar{\partial}_{\diamond} K_{v_{0}}(v)=\delta_{v v_{0}} f(v), \\
& \frac{1}{2 \pi i} \oint_{P_{Q}} f K_{v_{0}} d z=\frac{1}{\pi} \operatorname{ar}\left(F_{Q}\right) \bar{\partial}_{\Lambda} f(Q) K_{v_{0}}(Q)=0 .
\end{aligned}
$$

Here, we used that the value of the product on $\left[Q^{\prime}, v^{\prime}\right] \in E\left(X_{0}\right)$ is $f\left(v^{\prime}\right) K_{v_{0}}\left(Q^{\prime}\right)$, so in the first integration we could factor out $f(v)$, in the second one $K_{v_{0}}(Q)$.

By definition, the discrete contour $C_{v_{0}}$ is the oriented boundary of a topological disk in $F\left(X_{0}\right)$, so we can decompose the integration along $C_{v_{0}}$ into a couple of integrations along discrete elementary cycles $P_{v}$ and $P_{Q}$ as above. Summing up, only the contribution of $P_{v_{0}}$ is nonvanishing, and we get the desired result. The second formula is shown in an analog fashion.

Remark In the case of rhombic quad-graphs, Mercat formulated a discrete Cauchy's integral formula for the average of a discrete holomorphic function on $V(\Lambda)$ along an edge of $\Lambda$. In [6], Chelkak and Smirnov provided a discrete Cauchy's integral formula for discrete holomorphic functions on $V(\diamond)$ using an integration along cycles on $\Gamma$ and $\Gamma^{*}$, see Sect.2.6.1.

Theorem 2.36 Let $f: V\left(\Lambda_{0}\right) \rightarrow \mathbb{C}$ be discrete holomorphic, let $Q_{0} \in V\left(\nabla_{0}\right)$, and let $K_{Q_{0}}: V\left(\Lambda_{0}\right) \rightarrow \mathbb{C}$ be a discrete Cauchy's kernel with respect to $Q_{0}$ on $V\left(\Lambda_{0}\right)$.

Then, for any discrete contour $C_{Q_{0}}$ in $X_{0}$ surrounding $Q_{0}$ once in counterclockwise order that does not contain any edge $\left[Q_{0}, v\right]$ of $X_{0}, v \sim Q_{0}$ (see Fig. 5), the discrete Cauchy's integral formula is true:

$$
\partial_{\Lambda} f\left(Q_{0}\right)=-\frac{1}{2 \pi i} \oint_{C_{Q_{0}}} f \partial_{\Lambda} K_{Q_{0}} d z
$$

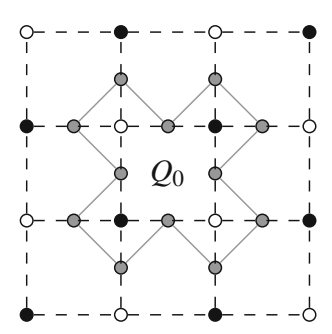

Fig. 5 Discrete contour as in Theorem 2.36 
Proof Let $D$ be the discrete domain in $F\left(X_{0}\right)$ bounded by $C_{Q_{0}}$. Since no edge of $C_{Q_{0}}$ passes through $Q_{0}$, the discrete one-form $\bar{\partial}_{\Lambda} K_{Q_{0}} d \bar{z}$ vanishes on $C_{Q_{0}}$. Therefore, $d K_{Q_{0}}=\partial_{\Lambda} K_{Q_{0}} d z$ on $C_{Q_{0}}$ and

$$
\oint_{C_{Q_{0}}} f \partial_{\Lambda} K_{Q_{0}} d z=\oint_{C_{Q_{0}}} f d K_{Q_{0}}=\iint_{D} d\left(f d K_{Q_{0}}\right)=\iint_{D} d f \wedge d K_{Q_{0}}
$$

due to discrete Stokes' Theorem 2.9 in the second equality and Theorem 2.16 that assures that $d\left(f d K_{Q_{0}}\right)=d f \wedge d K_{Q_{0}}+f d d K_{Q_{0}}$ and Proposition 2.10 that assures that $d d K_{Q_{0}}=0$ in the third equality. Now, $f$ is discrete holomorphic, so we obtain $d f \wedge d K_{Q_{0}}=\partial_{\Lambda} f \bar{\partial}_{\Lambda} K_{Q_{0}} \Omega_{\diamond}$. But $\bar{\partial}_{\Lambda} K_{Q_{0}}$ vanishes on all vertices of $\diamond_{0}$ but $Q_{0}$. Finally,

$$
-\frac{1}{2 \pi i} \oint_{C_{Q_{0}}} f \partial_{\Lambda} K_{Q_{0}} d z=-\frac{1}{2 \pi i} \iint_{F_{Q_{0}}} \partial_{\Lambda} f \bar{\partial}_{\Lambda} K_{Q_{0}} \Omega_{\diamond}=\partial_{\Lambda} f\left(Q_{0}\right) .
$$

Remark In general, there exists no analog of the above Theorem 2.36 for the discrete derivative of a discrete holomorphic function on $V\left(\nabla_{0}\right)$, because the discrete derivative itself does not need to be discrete holomorphic. However, in the special case of integer lattices, any discrete derivative of a discrete holomorphic function is itself discrete holomorphic. In Sect. 3.5, we will obtain discrete analogs of Cauchy's integral formulae for higher derivatives of discrete holomorphic functions.

\subsubsection{A Different Notation}

Let $W$ be a cycle on the edges of $\Gamma^{*}$, having (ordered) white vertices $w_{0}, w_{1}, \ldots, w_{m}$, $w_{m}=w_{0}$. Then, any edge connecting two consecutive vertices $w_{k}, w_{k+1}$ forms the diagonal of a quadrilateral face $Q\left(w_{k}, w_{k+1}\right) \in V(\diamond)$. We denote the set of such faces together with the induced orientation of their white diagonals by $W_{\diamond}$. For $Q \in W_{\diamond}$, we denote its white vertices by $w_{-}(Q), w_{+}(Q)$ such that the corresponding oriented diagonal goes from $w_{-}(Q)$ to $w_{+}(Q)$. Its black vertices are denoted by $b(Q), b^{\prime}(Q)$ in such a way that $w_{-}(Q), b(Q), w_{+}(Q), b^{\prime}(Q)$ appear in counterclockwise order. The reason why we do not choose our previous notation of Fig. 1 is that black and white vertices now play a different role that shall be indicated by the notation.

Now, we construct a cycle $B$ on the edges of $\Gamma$ having (ordered) black vertices $b_{0}, b_{1}, \ldots, b_{n}, b_{n}=b_{0}$, in the following way. We start with $b_{0}:=b\left(Q\left(w_{0}, w_{1}\right)\right)$. In the star of the vertex $w_{1}$, there are two simple paths on $\Gamma$ connecting $b_{0}$ and $b\left(Q\left(w_{1}, w_{2}\right)\right)$, and we choose the path that does not go through $Q\left(w_{0}, w_{1}\right)$. Note that it may happen that $b\left(Q\left(w_{1}, w_{2}\right)\right)=b_{0}$; in this case, we do not add any vertices to $B$. Also, $w_{2}=w_{0}$ is possible, which causes adding the nondirect path connecting the black vertices $b_{0}$ and $b\left(Q\left(w_{1}, w_{2}\right)\right)=b^{\prime}\left(Q\left(w_{0}, w_{1}\right)\right)$. 
Continuing this procedure till we have connected $b\left(Q\left(w_{m-1}, w_{m}\right)\right)$ with $b_{0}$, we end up with a closed path $B$ on $\Gamma$. Without loss of generality, any two consecutive vertices in $B$ are different. As above, any edge connecting two consecutive vertices $b_{k}, b_{k+1}$ forms the diagonal of a face $Q\left(b_{k}, b_{k+1}\right) \in V(\diamond)$. We denote the set of such faces together with the induced orientation of their black diagonals by $B_{\diamond}$. For $Q \in B_{\diamond}$, we denote its black vertices by $b_{-}(Q), b_{+}(Q)$ such that the corresponding oriented diagonal goes from $b_{-}(Q)$ to $b_{+}(Q)$. Finally, its white vertices are denoted by $w(Q), w^{\prime}(Q)$ in such a way that $b_{-}(Q), w^{\prime}(Q), b_{+}(Q), w(Q)$ appear in counterclockwise order.

Definition Let $W$ and $B$ be cycles as above and $h$ a function defined on $W_{\diamond} \cup B_{\diamond}$. We define the discrete integrals along $W$ and $B$ by

$$
\begin{aligned}
& \oint_{W} h(Q) d z:=\sum_{k=0}^{m-1} h\left(Q\left(w_{k}, w_{k+1}\right)\right)\left(w_{k+1}-w_{k}\right), \\
& \oint_{W} h(Q) d \bar{z}:=\sum_{k=0}^{m-1} h\left(Q\left(w_{k}, w_{k+1}\right)\right) \overline{\left(w_{k+1}-w_{k}\right)} \\
& \left.\oint_{B} h(Q) d z:=\sum_{k=0}^{n-1} h\left(Q\left(b_{k}, b_{k+1}\right)\right)\right)\left(b_{k+1}-b_{k}\right) \\
& \oint_{B} h(Q) d \bar{z}:=\sum_{k=0}^{n-1} h\left(Q\left(b_{k}, b_{k+1}\right)\right) \overline{\left(b_{k+1}-b_{k}\right)}
\end{aligned}
$$

In between the closed paths $B$ and $W$, there is a cycle $P$ on the medial graph $X$ that comprises exactly all edges $[Q, v]$ with $Q \in W_{\diamond}$ and $v \in B$ incident to $Q$ and all edges $[Q, v]$ with $Q \in B_{\diamond}$ and $v \in W$ incident to $Q$. The orientation of $[Q, v]$ is induced by the orientation of the corresponding parallel white or black diagonal. Figure 6 gives an example for this construction, where all cycles are oriented counterclockwise.

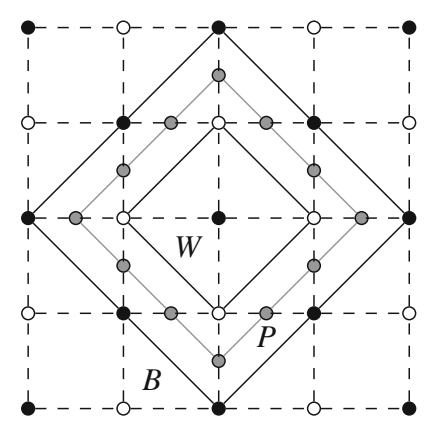

Fig. 6 Cycles $W$ on $\Gamma^{*}, B$ on $\Gamma$, and closed path $P$ on $X$ in between 
Remark Note that an oriented cycle $P$ on $X$ induces a white cycle $W=W(P)$ and a black cycle $B=B(P)$ in such a way that $W, P$, and $B$ are related as above.

Lemma 2.37 Let $P$ be an oriented cycle on $X$ and let $W=W(P)$ and $B=B(P)$ be the white and black cycles it induces. Let $f$ be a function defined on the vertices of $W$ and $B$ and $h$ a function defined on $W_{\diamond} \cup B_{\diamond}$. Then,

$$
\oint_{W} f(b(Q)) h(Q) d z+\oint_{B} f(w(Q)) h(Q) d z=2 \oint_{P} f h d z .
$$

Proof Any edge $e=[Q, b(Q)]\left(Q \in W_{\diamond}\right)$ or $[Q, w(Q)]\left(Q \in B_{\diamond}\right)$ of $P$ is parallel to either an edge $w_{-}(Q) w_{+}(Q)$ of $W$ or to an edge $b_{-}(Q) b_{+}(Q)$ of $B$, respectively, and vice versa. Since the complex number associated to $e$ is just half of the corresponding parallel edge of $\Gamma$ or $\Gamma^{*}, 2 \int_{e} f h=f(b(Q)) h(Q)\left(w_{+}(Q)-w_{-}(Q)\right)$ in the first and $2 \int_{e} f h=f(w(Q)) h(Q)\left(b_{+}(Q)-b_{-}(Q)\right)$ in the second case. Therefore, the discrete integral along $P$ decomposes into one along $B$ and one along $W$.

Remark Note that the construction of $B$ and Lemma 2.37 are also valid if $W$ consists of a single point or of only two edges (being the same, but traversed in both directions). In both cases, $P$ will be a discrete contour, as well when $W$ is simple and oriented counterclockwise.

The discrete Cauchy's integral formula of Chelkak and Smirnov in [6] reads as

$$
\pi i h\left(Q_{0}\right)=\oint_{W} h(Q) K_{Q_{0}}(b(Q)) d z+\oint_{B} h(Q) K_{Q_{0}}(w(Q)) d z
$$

if $Q_{0} \in V(\diamond)$ is surrounded once by the simple closed curve $W$ on $\Gamma^{*}$ in counterclockwise direction, $h$ is discrete holomorphic on $V(\diamond)$, and $K_{Q_{0}}: V(\Lambda) \rightarrow \mathbb{C}$ is a discrete Cauchy's kernel with respect to $Q_{0}$. The above Lemma 2.37 directly relates this formulation to the one of the discrete Cauchy's integral formula in Theorem 2.35.

Finally, we conclude this section with a proposition relying on the decomposition of a discrete contour into black and white cycles. In Proposition 2.13(i), we have already seen that $f d g+g d f$ is closed for functions $f, g: V(\Lambda) \rightarrow \mathbb{C}$. Actually, a slightly stronger statement is true:

Proposition 2.38 Let $W$ be a closed cycle on $\Gamma^{*}$ and $B$ be the corresponding closed cycle on $\Gamma$ as above, and let $f, g: V(\Lambda) \rightarrow \mathbb{C}$. Then,

$$
\oint_{W} f(b(Q))\left(\partial_{\Lambda} g(Q) d z+\bar{\partial}_{\Lambda} g(Q) d \bar{z}\right)+\oint_{B} g(w(Q))\left(\partial_{\Lambda} f(Q) d z+\bar{\partial}_{\Lambda} f(Q) d \bar{z}\right)=0 .
$$

Proof We first rewrite the discrete integral along the cycle $W$. By definition, we have $d g=\partial_{\Lambda} g d z+\bar{\partial}_{\Lambda} g d \bar{z}$. By discrete Stokes' Theorem 2.9, $\int_{e} d g=g\left(w_{+}\right)-g\left(w_{-}\right)$ if $e$ is an edge on $X$ parallel to $w_{-} w_{+}$and oriented the same, so 


$$
\begin{aligned}
\oint_{W} f(b(Q))\left(\partial_{\Lambda} g(Q) d z+\bar{\partial}_{\Lambda} g(Q) d \bar{z}\right) & =\sum_{Q \in W_{\diamond}} f(b(Q))\left(g\left(w_{+}(Q)\right)-g\left(w_{-}(Q)\right)\right) \\
& =-\sum_{Q \in B_{\diamond}} g(w(Q))\left(f\left(b_{+}(Q)\right)-f\left(b_{-}(Q)\right)\right),
\end{aligned}
$$

where we changed the summation along the path $W$ into a summation along the path $B$ in the last step. Similar to above, we rewrite the discrete integral along $B$ as

$$
\oint_{B} g(w(Q))\left(\partial_{\Lambda} f(Q) d z+\bar{\partial}_{\Lambda} f(Q) d \bar{z}\right)=\sum_{Q \in B_{\diamond}} g(w(Q))\left(f\left(b_{+}(Q)\right)-f\left(b_{-}(Q)\right)\right) .
$$

In summary,

$$
\oint_{W} f(b(Q))\left(\partial_{\Lambda} g(Q) d z+\bar{\partial}_{\Lambda} g(Q) d \bar{z}\right)+\oint_{B} g(w(Q))\left(\partial_{\Lambda} f(Q) d z+\bar{\partial}_{\Lambda} f(Q) d \bar{z}\right)=0 .
$$

\section{Discrete Complex Analysis on Planar Parallelogram-Graphs}

\subsection{Preliminaries}

Definition A planar parallelogram-graph $\Lambda$ (without boundary) is a planar quadgraph without boundary such that all its faces are parallelograms. Its dual $\diamond$ is embedded in the complex plane by placing all $Q \in V(\diamond)$ at the center $\hat{Q}$ of the corresponding parallelogram $Q$ and connecting adjacent vertices by straight line segments.

Remark Remembering the duality $V(\diamond) \cong F(\Lambda)$, we will omit the hat above $Q$ in the sequel and identify the vertex $Q \in V(\diamond)$ representing a parallelogram in $\Lambda$ with the corresponding point $\hat{Q}$ in the complex plane. It will be clear from the context whether $Q$ is meant to be a face of $\Lambda$ or a point in the complex plane.

In Sect. 3, discrete complex analysis on planar parallelogram-graphs $\Lambda$ is discussed. As in Sect. 2, $\Lambda$ is bipartite, the induced graphs on black and white vertices are denoted by $\Gamma$ and $\Gamma^{*}$, respectively, and we assume that the cellular decomposition induced by $\Lambda$ is locally finite and strongly regular (which already follows from all faces being parallelograms).

In Propositions 2.1, 2.5, and 2.22, we have already seen that discrete complex analysis on parallelogram-graphs is closer to the classical theory than on general quad-graphs. For example, $f(v)=v^{2}$ is a discrete holomorphic function on $V(\Lambda)$ and $\partial_{\Lambda} f(Q)=2 Q ; h(Q)=Q$ is a discrete holomorphic function on $V(\diamond)$ and $\partial_{\diamond} h \equiv 1$; and the discrete Laplacian $\triangle$ approximates the smooth one correctly up to order two. 
In order to concentrate on the calculation of the asymptotics of a certain discrete Green's function and discrete Cauchy's kernels, we postpone the discussion of some necessary combinatorial and geometric results on planar parallelogram-graphs to the appendix. Our setup is closely related to the quasicrystallic parallelogram-graphs discussed in [2]. There, the quad-graph was embedded into $\mathbb{Z}^{n}$ and explicit formulae for a discrete exponential function and a discrete logarithm were introduced. These results relied on Kenyon's formulae for a discrete exponential and a discrete Green's function in [16].

In the following, the ideas of [2] are adapted to our slightly more general setting in Sect. 3.2. The discrete exponential is the basic building block for the integral formulae of a discrete Green's function in Sect. 3.3 and discrete Cauchy's kernels in Sect. 3.4. The corresponding functions can be defined for general planar parallelogram-graphs, but we need more regularity of the graph to calculate their asymptotics. The two conditions we use are that all interior angles of the parallelograms are bounded (the same condition was used by Chelkak and Smirnov in [6]) and that the ratio of side lengths of the parallelograms is bounded as well. For rhombic quad-graphs, the second condition is trivially fulfilled; for quasicrystallic graphs, there are only a finite number of interior angles. Note that instead of using boundedness of the ratio of side lengths of the parallelograms, we can assume that the side lengths themselves are bounded. This seems to be a stronger condition at first, but actually, both conditions are equivalent, see Proposition 4.3 in the appendix.

Remark To our knowledge, it is an open problem to find an explicit formula for a discrete Green's function or a discrete Cauchy's kernel in the case of general quadgraphs as discussed in Sect. 2.

We conclude this section by a discussion of integer lattices in Sect. 3.5. On these graphs, discrete holomorphic functions can be discretely differentiated infinitely many times, and for all higher order discrete derivatives, discrete Cauchy's integral formulae with the asymptotics one expects from the asymptotics of the discrete Cauchy's integral formula for a discrete holomorphic function hold true.

Lemma 3.1 Let $v, v^{\prime} \in V(\Lambda)$, let $k$ be an odd integer, let $e_{1}, \ldots, e_{n}$ be a sequence of $n$ oriented edges of $\Lambda$ forming a directed path from $v$ to $v^{\prime}$ on $\Lambda$, and let $f_{1}, \ldots, f_{m}$ be another such sequence of $m$ oriented edges. Then,

$$
\sum_{j=1}^{n} e_{j}^{k}=\sum_{j=1}^{m} f_{j}^{k}
$$

Here, the calculations are performed directly with edges rather than replacing them with their associated complex numbers (see Sect.2.1.1).

Proof Consider a path $p_{1}, p_{2}, p_{3}, p_{4}$ of oriented edges of $\Lambda$ going once around a parallelogram. Since $p_{1}=-p_{3}$ and $p_{2}=-p_{4}$, we have $\sum_{j=1}^{4} p_{j}^{k}=0$. Now, any closed cycle on the planar graph $\Lambda$ can be decomposed into elementary oriented cycles around faces, where edges $e,-e$ with opposite orientation cancel out, and 
pairs of oppositely oriented edges (that are not necessarily successive). Using that $e^{k}+(-e)^{k}=0$, the claim now follows from

$$
\sum_{j=1}^{n} e_{j}^{k}+\sum_{j=1}^{m}\left(-f_{j}\right)^{k}=0
$$

for the cycle $e_{1}, \ldots, e_{n},-f_{1}, \ldots,-f_{m}$ of oriented edges starting and ending in $v$.

In the remainder of the paper, we use the following shorthand notation.

Definition Let $v, v^{\prime} \in V(\Lambda)$ and $Q, Q^{\prime} \in V(\diamond)$.

(i) Choose any directed path of edges $e_{1}, \ldots, e_{n}$ on $\Lambda$ going from $v^{\prime}$ to $v$. Define

$$
J\left(v, v^{\prime}\right):=\sum_{j=1}^{n} e_{j}^{-1}
$$

which does not depend on the choice of path from $v$ to $v^{\prime}$ due to Lemma 3.1.

(ii) Choose any vertex $v_{Q}$ incident to $Q$ and any directed path of edges $e_{1}, \ldots, e_{n}$ on $\Lambda$ from $v$ to $v_{Q}$. Moreover, let $d_{1}, d_{2}$ be the two oriented edges of $Q$ that emanate in $v_{Q}$. We now define

$$
-J(v, Q)=J(Q, v):=\sum_{j=1}^{n} e_{j}^{-1}+\frac{1}{2} d_{1}^{-1}+\frac{1}{2} d_{2}^{-1} .
$$

Note that $J(Q, v)$ does not depend on the choice of path from $v$ to $v_{Q}$ by Lemma 3.1 nor on the choice of $v_{Q}$ by a similar argument as in the proof of the above lemma.

Moreover, let $\tau(v, Q)=\tau(Q, v):=1 /\left(d_{1} d_{2}\right)$ if $v_{Q}, v$ are both in $V(\Gamma)$ or both in $V\left(\Gamma^{*}\right)$ and $\tau(v, Q)=\tau(Q, v):=-1 /\left(d_{1} d_{2}\right)$ otherwise. Since $Q$ is a parallelogram, these quantities depend on $v$ and $Q$ only.

(iii) Choose any vertices $v_{Q^{\prime}}$ incident to $Q^{\prime}$ and $v_{Q}$ incident to $Q$ and a directed path of edges $e_{1}, \ldots, e_{n}$ on $\Lambda$ going from $v_{Q^{\prime}}$ to $v_{Q}$. Let $d_{1}, d_{2}$ be the two oriented edges of $Q^{\prime}$ ending in $v_{Q^{\prime}}$ and $f_{1}, f_{2}$ the two oriented edges of $Q$ emanating from $v_{Q}$. Define

$$
J\left(Q, Q^{\prime}\right):=\frac{1}{2} d_{1}^{-1}+\frac{1}{2} d_{2}^{-1}+\sum_{j=1}^{n} e_{j}^{-1}+\frac{1}{2} f_{1}^{-1}+\frac{1}{2} f_{2}^{-1}
$$

$J\left(Q, Q^{\prime}\right)$ does not depend on the choice of $v_{Q^{\prime}}$ and $v_{Q}$ or the path from $v_{Q^{\prime}}$ to $v_{Q}$.

Furthermore, let $\tau\left(Q, Q^{\prime}\right):=1 /\left(d_{1} d_{2} f_{1} f_{2}\right)$ if $v_{Q}, v_{Q^{\prime}}$ are both in $V(\Gamma)$ or both in $V\left(\Gamma^{*}\right)$ and $\tau\left(Q, Q^{\prime}\right):=-1 /\left(d_{1} d_{2} f_{1} f_{2}\right)$ otherwise. $\tau\left(Q, Q^{\prime}\right)$ depends on $Q$ and $Q^{\prime}$ only, since they are both parallelograms. 
Remark In the case that all parallelograms are rhombi of side length one, we have $J\left(x, x^{\prime}\right)=\overline{x-x^{\prime}}$.

Finally, the notion of the argument of a complex number will become important in the sequel. In our paper, it will be usually an arbitrary real number and not a number modulo $2 \pi$.

Definition For a complex number $\lambda \neq 0$, a real number $\phi$ such that $\lambda / \exp (i \phi)$ is a positive real number is called argument of $\lambda$. On the other hand, $\arg (\lambda)$ denotes the unique argument $\phi$ of $\lambda$ that is contained in the interval $(-\pi, \pi]$.

\subsection{Discrete Exponential Function}

Definition Let $v_{0}$ be a vertex of $\Lambda$. Then, the discrete exponentials $\mathrm{e}\left(\lambda, \cdot ; v_{0}\right)$ and $\exp \left(\lambda, \cdot ; v_{0}\right)$ on $V(\Lambda)$ are the rational functions in the complex variable $\lambda$ that are inductively defined by $\mathrm{e}\left(\lambda, v_{0} ; v_{0}\right)=1=\exp \left(\lambda, v_{0} ; v_{0}\right)$ and

$$
\frac{\mathrm{e}\left(\lambda, v^{\prime} ; v_{0}\right)}{\mathrm{e}\left(\lambda, v ; v_{0}\right)}=\frac{\lambda+\left(v^{\prime}-v\right)}{\lambda-\left(v^{\prime}-v\right)} \quad \text { and } \quad \frac{\exp \left(\lambda, v^{\prime} ; v_{0}\right)}{\exp \left(\lambda, v ; v_{0}\right)}=\frac{1+\frac{\lambda}{2}\left(v^{\prime}-v\right)}{1-\frac{\lambda}{2}\left(v^{\prime}-v\right)}
$$

for all vertices $v, v^{\prime} \in V(\Lambda)$ adjacent to each other.

Remark Note that the quotient of e or exp at the vertices of an oriented edge $e$ is by definition the inverse of the quotient for the edge $-e$ oriented in the opposite direction. Since all faces of $\Lambda$ are parallelograms, the complex numbers associated to opposite edges oriented the same are equal and therefore are corresponding quotients of the discrete exponentials. Thus, the discrete exponentials are well-defined.

For $v \in V(\Lambda), \exp \left(\cdot, v ; v_{0}\right)$ is a rational function on $\mathbb{C}$ with poles being the complex numbers associated to the oriented edges of a shortest directed path connecting $v_{0}$ with $v$. It follows from Lemma 4.2 in the appendix that the arguments of all poles can be chosen to lie in an interval of length less than $\pi$. If in addition the interior angles of parallelograms are bounded from below by $\alpha_{0}$, then the arguments of all poles can be chosen to lie even in an interval of length at most $\pi-\alpha_{0}$.

Remark Note that $\exp \left(\lambda, \cdot ; v_{0}\right)=\mathrm{e}\left(2 / \lambda, \cdot ; v_{0}\right)$. Hence, e and exp are equivalent up to reparametrization. On square lattices, the discrete exponential was already considered by Ferrand [12] and Duffin [9]. The discrete exponential e on rhombic lattices was used in the papers $[2,5,16]$. To be comparable to these, we use e and not exp to perform our calculations of the asymptotic behavior. In contrast, Mercat [20], Chelkak and Smirnov [6] preferred the parametrization of exp that is closer to the smooth setting. Indeed, Mercat remarked that the discrete exponential exp in the rhombic setting is a generalization of the formula 


$$
\exp (\lambda x)=\left(\frac{1+\frac{\lambda x}{2 n}}{1-\frac{\lambda x}{2 n}}\right)^{n}+O\left(\frac{\lambda^{3} x^{3}}{n^{2}}\right)
$$

to the case when the path from the origin to $x$ consists of $O(|x| / \delta)$ straight line segments of length $\delta$ of any directions [20].

Definition For a face $Q \in V(\diamond)$ with incident vertices $v_{-}, v_{-}^{\prime}, v_{+}, v_{+}^{\prime}$ in counterclockwise order and $v \in V(\Lambda)$, we define the discrete exponentials as the following rational functions in the complex variable $\lambda$ :

$$
\begin{aligned}
\mathrm{e}(\lambda, v ; Q) & :=\frac{\mathrm{e}\left(\lambda, v ; v_{ \pm}\right)}{\left(\lambda-\left(v_{ \pm}-v_{+}^{\prime}\right)\right)\left(\lambda-\left(v_{ \pm}-v_{-}^{\prime}\right)\right)}, \\
\exp (\lambda, Q ; v) & :=\frac{\exp \left(\lambda, v_{ \pm} ; v\right)}{\left(1-\frac{\lambda}{2}\left(v_{+}^{\prime}-v_{ \pm}\right)\right)\left(1-\frac{\lambda}{2}\left(v_{-}^{\prime}-v_{ \pm}\right)\right)} .
\end{aligned}
$$

Remark For arbitrary $Q_{0} \in V(\diamond)$ and $v_{0} \in V(\Lambda)$, the above definition yields welldefined rational functions $\mathrm{e}\left(\cdot, v_{0} ; Q_{0}\right)$ and $\exp \left(\cdot, Q_{0} ; v_{0}\right)$. As long as $\lambda$ is not a pole, $\mathrm{e}\left(\lambda, \cdot ; Q_{0}\right)$ is a function on $V(\Lambda)$ and $\exp \left(\lambda, \cdot ; v_{0}\right)$ is a function on $V(\diamond)$.

Proposition 3.2 Let $v_{0} \in V(\Lambda), Q \in V(\diamond)$. Then, for any $\lambda \in \mathbb{C}$ that is not a pole of $\exp \left(\cdot, v ; v_{0}\right)$ for any vertex $v \sim Q, \exp \left(\lambda, \cdot ; v_{0}\right)$ is discrete holomorphic at $Q$ and

$$
\left(\partial_{\Lambda} \exp \left(\lambda, \cdot ; v_{0}\right)\right)(Q)=\lambda \exp \left(\lambda, Q ; v_{0}\right)
$$

Proof Let $v_{-}, v_{-}^{\prime}, v_{+}, v_{+}^{\prime}$ be the vertices of $Q$ in counterclockwise order. Let us denote $a:=v_{+}^{\prime}-v_{-}$and $b:=v_{-}^{\prime}-v_{-}$. Using Lemma 2.3 that describes $\bar{\partial}_{\Lambda}$ as a discrete contour integration, $v_{+}-v_{-}=a+b, v_{+}^{\prime}-v_{-}^{\prime}=a-b$, and the inductive formula for $\exp$, we get that $\left(\bar{\partial}_{\Lambda} \exp \left(\lambda, \cdot ; v_{0}\right)\right)(Q)$ equals

$$
\begin{aligned}
& \frac{\exp \left(\lambda, v_{-} ; v_{0}\right)}{2 i \operatorname{ar}\left(F_{Q}\right)} \oint_{\partial F_{Q}} \frac{\exp \left(\lambda, \cdot ; v_{0}\right)}{\exp \left(\lambda, v_{-} ; v_{0}\right)} d z \\
= & \frac{\exp \left(\lambda, v_{-} ; v_{0}\right)}{2 i \operatorname{ar}\left(F_{Q}\right)}\left(\frac{a+b}{2} \cdot\left(\frac{1+\frac{\lambda}{2} b}{1-\frac{\lambda}{2} b}-\frac{1+\frac{\lambda}{2} a}{1-\frac{\lambda}{2} a}\right)+\frac{a-b}{2} \cdot\left(\frac{1+\frac{\lambda}{2} b}{1-\frac{\lambda}{2} b} \frac{1+\frac{\lambda}{2} a}{1-\frac{\lambda}{2} a}-1\right)\right) \\
= & \frac{\exp \left(\lambda, v_{-} ; v_{0}\right)}{4 i \operatorname{ar}\left(F_{Q}\right)\left(1-\frac{\lambda}{2} a\right)\left(1-\frac{\lambda}{2} b\right)}((a+b) \lambda(b-a)+(a-b) \lambda(a+b))=0 .
\end{aligned}
$$

Here, $F_{Q}$ is the Varignon parallelogram inside $Q$, and $\operatorname{ar}\left(F_{Q}\right)=\operatorname{area}(Q)$. Similarly, we obtain that $\left(\partial_{\Lambda} \exp \left(\lambda, \cdot ; v_{0}\right)\right)(Q)$ equals

$$
\begin{aligned}
& \frac{\exp \left(\lambda, v_{-} ; v_{0}\right)}{4 i \operatorname{ar}\left(F_{Q}\right)\left(1-\frac{\lambda}{2} a\right)\left(1-\frac{\lambda}{2} b\right)}(-\overline{(a+b)} \lambda(b-a)+\overline{(b-a)} \lambda(a+b)) \\
= & \frac{\lambda \exp \left(\lambda, v_{-} ; v_{0}\right)}{2 i|a||b| \sin \left(\varphi_{Q}\right)\left(1-\frac{\lambda}{2} a\right)\left(1-\frac{\lambda}{2} b\right)} 2 i \operatorname{Im}(a \bar{b})=\lambda \exp \left(\lambda, Q ; v_{0}\right) .
\end{aligned}
$$




\subsection{Asymptotics of the Discrete Green's Function}

Following the presentation in [2], we first define a discrete logarithmic function on the lift $\tilde{\Lambda}_{v_{0}}$ of the quad-graph $\Lambda$ onto the Riemann surface of the logarithmic function $\log \left(\cdot-v_{0}\right)$, which can be considered as a branched covering of the quad-graph $\Lambda$ seen as a cellular decomposition of the complex plane.

Throughout the following paragraphs, fix $v_{0} \in V(\Lambda)$, and let $e_{1}, e_{2}, \ldots, e_{n}$ be the directed edges starting in $v_{0}$, ordered according to their slopes.

Definition To each of these edges $e$ we assign the angle $\theta_{e}:=\arg (e) \in(-\pi, \pi]$. We assume that $\theta_{e_{1}}<\theta_{e_{n}}$. Now, define $\theta_{a+b n}:=\theta_{a}+2 \pi b$, where $a \in\{1, \ldots, n\}$ and $b \in \mathbb{Z}$. For $m \in \mathbb{Z}$, let $e_{m}:=e_{m \bmod n}$, considering the residue classes $\{1,2, \ldots, n\}$.

Definition Let $e$ be one of the $e_{k}$. The sector $U_{e} \subset \Lambda$ is the subgraph of $\Lambda$ that consists of all vertices and edges of directed paths on $\Lambda$ starting in $v_{0}$ whose oriented edges have arguments that can be chosen to lie in $[\arg (e), \arg (e)+\pi)$.

Definition For $m \in \mathbb{Z}$, we define the graph $\tilde{U}_{m}$ to be the sector $U_{e_{m}}$ with the additional data that each vertex $v$ of $U_{e_{m}}$ besides $v_{0}$ is assigned the real number $\vartheta_{m}(v)$ given by $\vartheta_{m}(v) \equiv \arg \left(v-v_{0}\right) \bmod 2 \pi$ and $\vartheta_{m}(v) \in\left[\theta_{m}, \theta_{m}+\pi\right)$. Then,

$$
\tilde{U}:=\bigcup_{m=-\infty}^{\infty} \tilde{U}_{m}
$$

defines a graph $\tilde{\Lambda}_{v_{0}}$ on the Riemann surface of $\log \left(\cdot-v_{0}\right)$ that projects to the planar parallelogram graph $\Lambda$. Here, vertices $v$ of $U_{e_{m}}$ and $v^{\prime}$ of $U_{e_{m^{\prime}}}$ are equal as vertices of $\tilde{U}_{m}$ and $\tilde{U}_{m^{\prime}}$ if and only if $v=v^{\prime}$ and either $v=v^{\prime}=v_{0}$ or $\vartheta_{m}(v)=\vartheta_{m^{\prime}}\left(v^{\prime}\right)$.

Remark Apart from the additional data of the vertices, $\tilde{U}_{m}$ is composed of all the vertices of edges of directed paths of edges on $\Lambda$ starting in $v_{0}$ whose arguments can be chosen to lie in $\left[\theta_{m}, \theta_{m}+\pi\right)$. It follows that all $\tilde{U}_{m+b n}, b \in \mathbb{Z}$ and $1 \leqslant m \leqslant n$, cover the same sector $U_{e_{m}}$, and $\tilde{U}_{m} \cap \tilde{U}_{m^{\prime}}$ contains more than just $v_{0}$ if and only if $\left|m-m^{\prime}\right|<n$. In addition, Lemma 4.2 shows that the union of all $U_{e_{k}}, k=1, \ldots, n$, covers the whole quad-graph $\Lambda$. It follows that $\tilde{\Lambda}_{v_{0}}$ is a branched covering of the cellular decomposition $\Lambda$, branched over $v_{0}$.

Definition To each vertex $\tilde{v} \in V\left(\tilde{\Lambda}_{v_{0}}\right)$ covering a vertex $v \neq v_{0}$ of $\Lambda$, let us denote $\theta_{\tilde{v}}:=\vartheta_{m}(v)$ if $\tilde{v} \in \tilde{U}_{m}$.

Remark $\theta_{\tilde{v}}$ increases by $2 \pi$ when the vertex winds once around $v_{0}$ in counterclockwise order; and if $\tilde{v}, \tilde{v}^{\prime} \neq v_{0}$ are adjacent vertices of $\tilde{\Lambda}_{v_{0}}$, then $\left|\theta_{\tilde{v}}-\theta_{\tilde{v}^{\prime}}\right|<\pi$.

Note that by construction, if we connect $v_{0}$ to some $\tilde{v} \neq v_{0}$ by a shortest directed path of edges of $\tilde{\Lambda}_{v_{0}}$, then the arguments of all oriented edges can be chosen to lie all in $\left(\theta_{\tilde{v}}-\pi, \theta_{\tilde{v}}+\pi\right)$. 
Definition Let $v_{0} \in V(\Lambda)$ and let $\tilde{\Lambda}_{v_{0}}$ be the corresponding branched covering of $\Lambda$. The discrete logarithmic function on $V\left(\tilde{\Lambda}_{v_{0}}\right)$ is given by $\log \left(v_{0} ; v_{0}\right):=0$ and

$$
\log \left(\tilde{v} ; v_{0}\right):=\frac{1}{2 \pi i} \int_{C_{\tilde{v}}} \frac{\log (\lambda)}{2 \lambda} \mathrm{e}\left(\lambda, v ; v_{0}\right) d \lambda
$$

for $\tilde{v} \neq v_{0}$. Here, $C_{\tilde{v}}$ is a collection of sufficiently small counterclockwise oriented loops going once around each pole of $\mathrm{e}\left(\cdot, v ; v_{0}\right), v \in V(\Lambda)$ being the projection of $\tilde{v} \in V\left(\tilde{\Lambda}_{v_{0}}\right)$. On each loop around a pole $e$, we take the branch of logarithm that satisfies $\operatorname{Im}(\log (e)) \in\left(\theta_{\tilde{v}}-\pi, \theta_{\tilde{v}}+\pi\right)$.

Remark Let us suppose that $v_{0}$ is a black vertex. In the special case of a rhombic quasicrystallic quad-graph, the notion of the discrete logarithm is motivated as follows [2]: The discrete logarithm is real-valued and does not branch on black vertices; and it is purely imaginary on white points and increases by $2 \pi i$ if one goes once around $v_{0}$ in counterclockwise order. Therefore, the discrete logarithm models the behavior of the real and the imaginary part of the smooth logarithm if restricted to black and white vertices, respectively. As we will see later in the proof of Proposition 3.4, the values at vertices adjacent to $v_{0}$ coincide with the smooth logarithm.

Lemma 3.3 Let $\tilde{v}, \tilde{v}^{\prime} \in V\left(\tilde{\Lambda}_{v_{0}}\right)$ be two points that cover the same vertex $v \in V(\Lambda)$ such that $\theta_{\tilde{v}^{\prime}}-\theta_{\tilde{v}}=2 \pi$. Then

$$
\log \left(\tilde{v}^{\prime} ; v_{0}\right)-\log \left(\tilde{v} ; v_{0}\right)=0
$$

if $v_{0}, v$ are both in $V(\Gamma)$ or both in $V\left(\Gamma^{*}\right)$, and otherwise

$$
\log \left(\tilde{v}^{\prime} ; v_{0}\right)-\log \left(\tilde{v} ; v_{0}\right)=2 \pi i
$$

Proof By definition,

$$
\log \left(\tilde{v}^{\prime} ; v_{0}\right)-\log \left(\tilde{v} ; v_{0}\right)=\int_{C_{\tilde{v}}} \frac{1}{2 \lambda} \mathrm{e}\left(\lambda, v ; v_{0}\right) d \lambda
$$

The function that is integrated is meromorphic on $\mathbb{C}$ with poles given by the one of $\mathrm{e}\left(\cdot, v ; v_{0}\right)$ and zero. By residue formula, we can replace integration along $C_{\tilde{v}}$ by an integration along a circle centered at 0 with large radius $R$ (such that all other poles lie inside the disk) in counterclockwise direction and an integration along a circle centered at 0 with small radius $r$ (such that all poles lie outside the disk) in clockwise direction. Now, e $\left(\infty, v ; v_{0}\right)=1$. If $v_{0}, v$ are both in $V(\Gamma)$ or both in $V\left(\Gamma^{*}\right)$, then $\mathrm{e}\left(0, v ; v_{0}\right)=1$, otherwise $\mathrm{e}\left(0, v ; v_{0}\right)=-1$. Hence, $\log \left(\tilde{v}^{\prime} ; v_{0}\right)-\log \left(\tilde{v} ; v_{0}\right)=0$ in the first and $\log \left(\tilde{v}^{\prime} ; v_{0}\right)-\log \left(\tilde{v} ; v_{0}\right)=2 \pi i$ in the latter case.

In particular, the real part of the discrete $\operatorname{logarithm} \log \left(\cdot ; v_{0}\right)$ is a well-defined function on $V(\Lambda)$. Divided by $2 \pi$, one actually obtains a discrete Green's function 
with respect to $v_{0}$. In the rhombic case, it coincides with Kenyon's discrete logarithm in [16] as was shown in [2].

Proposition 3.4 Let $v_{0}$ be a vertex of $V(\Lambda)$. The function $G\left(\cdot ; v_{0}\right): V(\Lambda) \rightarrow \mathbb{R}$ defined by $G\left(v_{0} ; v_{0}\right)=0$ and

$$
G\left(v ; v_{0}\right)=\frac{1}{2 \pi} \operatorname{Re}\left(\frac{1}{2 \pi i} \int_{C_{v}} \frac{\log (\lambda)}{2 \lambda} e\left(\lambda, v ; v_{0}\right) d \lambda\right)
$$

for each $v \neq v_{0}$ is a (free) discrete Green's function with respect to $v_{0}$. Here, $C_{v}$ is a collection of sufficiently small counterclockwise oriented loops going once around each pole of $e\left(\cdot, v ; v_{0}\right)$, and on each loop around a pole $e$, we take the branch of $\log$ where $\operatorname{Im}(\log (e)) \in\left(\arg \left(v-v_{0}\right)-\pi, \arg \left(v-v_{0}\right)+\pi\right)$.

Proof When evaluating the real part of the integral, we can also take the branches of the $\log$ arithm that satisfy $\operatorname{Im}(\log (e)) \in\left(\arg \left(v-v_{0}\right)-\pi, \arg \left(v-v_{0}\right)+\pi\right)+2 k \pi$ for all poles $e$, where $k \in \mathbb{Z}$ is fixed. Indeed, Lemma 3.3 asserts that under this change, the discrete logarithm changes by 0 or $2 k \pi i$, so the real part does not change.

Consider all faces incident to $v_{0}$ in $\Lambda$ and its lift to $\tilde{\Lambda}_{v_{0}}$. For any vertex $v_{s}^{\prime} \in V(\Lambda)$ adjacent to $v_{0}, \lambda=v_{s}^{\prime}-v_{0}$ is the only pole of $\mathrm{e}\left(\lambda, v_{s}^{\prime} ; v_{0}\right)$. The residue theorem shows that $\log \left(\tilde{v}_{s}^{\prime} ; v_{0}\right)$ and $\log \left(v_{s}^{\prime}-v_{0}\right)$ coincide up to a multiple of $2 \pi i$ if $\tilde{v}_{s}^{\prime} \operatorname{covers} v_{s}^{\prime}$. By a similar calculation for vertices $v_{s}$ adjacent to $v_{0}$ in $\Gamma$ or $\Gamma^{*}$, we finally get

$$
\begin{aligned}
& G\left(v_{s}^{\prime} ; v_{0}\right)=\frac{1}{2 \pi} \operatorname{Re}\left(\log \left(v_{s}^{\prime}-v_{0}\right)\right), \\
& G\left(v_{s} ; v_{0}\right)=\frac{1}{2 \pi} \operatorname{Re}\left(\left(\log \left(v_{s}^{\prime}-v_{0}\right)-\log \left(v_{s-1}^{\prime}-v_{0}\right)\right) \frac{v_{s}-v_{0}}{v_{s}^{\prime}-v_{s-1}^{\prime}}\right),
\end{aligned}
$$

where $v_{0}, v_{s-1}^{\prime}, v_{s}, v_{s}^{\prime}$ are the vertices of $Q_{s} \sim v_{0}$ in counterclockwise order.

As in Corollary 2.20, let $\rho_{s}:=-i\left(v_{s}^{\prime}-v_{s-1}^{\prime}\right) /\left(v_{s}-v_{0}\right)$. In addition, we assign angles $\theta_{v_{s}^{\prime}} \equiv \arg \left(v_{s}^{\prime}-v_{0}\right) \bmod 2 \pi$ in such a way that $0<\theta_{v_{s}^{\prime}}-\theta_{v_{s-1}^{\prime}}<\pi$. Due to $\operatorname{Re}\left(-i / \rho_{s}\right)=-\operatorname{Im}\left(\rho_{s}\right) /\left|\rho_{s}\right|^{2}$ and $\operatorname{Im}\left(-i / \rho_{s}\right)=-\operatorname{Re}\left(\rho_{s}\right) /\left|\rho_{s}\right|^{2}$,

$$
\begin{aligned}
& \frac{\left|\rho_{s}\right|^{2}\left(G\left(v_{s} ; v_{0}\right)-G\left(v_{0} ; v_{0}\right)\right)+\operatorname{Im}\left(\rho_{s}\right)\left(G\left(v_{s}^{\prime} ; v_{0}\right)-G\left(v_{s-1}^{\prime} ; v_{0}\right)\right)}{\operatorname{Re}\left(\rho_{s}\right)} \\
= & \frac{\left|\rho_{s}\right|^{2} \operatorname{Re}\left(-i / \rho_{s}\right)+\operatorname{Im}\left(\rho_{s}\right)}{2 \pi \operatorname{Re}\left(\rho_{s}\right)} \log \left|\frac{v_{s}^{\prime}-v_{0}}{v_{s-1}^{\prime}-v_{0}}\right|-\frac{\left|\rho_{s}\right|^{2} \operatorname{Im}\left(-i / \rho_{s}\right)}{2 \pi \operatorname{Re}\left(\rho_{s}\right)}\left(\theta_{v_{s}^{\prime}}-\theta_{v_{s-1}^{\prime}}\right) \\
= & \frac{\theta_{v_{s}^{\prime}}-\theta_{v_{s-1}^{\prime}}}{2 \pi} .
\end{aligned}
$$

It follows from the explicit formula for the discrete Laplacian in Corollary 2.20 that $\triangle G\left(v_{0} ; v_{0}\right)=1 /\left(2 \operatorname{ar}\left(F_{v_{0}}\right)\right)$.

Now, we show that $G\left(\cdot ; v_{0}\right)$ is discrete harmonic away from $v_{0}$. For this, we consider the star of some vertex $v \neq v_{0}$, i.e., all faces of $\Lambda$ incident to $v \in V(\Lambda)$. 
Let us assume that we can find one collection $C$ of loops together with appropriate branches of $\log$ such that for all vertices $v^{\prime}$ of the star, $G\left(v^{\prime} ; v_{0}\right)$ can be computed by an integration along $C$ instead of $C_{v^{\prime}}$. Then, when we compute $\triangle G\left(v ; v_{0}\right)$, we can exchange the discrete Laplacian not only with the real part, but also with the integration. Since $\mathrm{e}\left(\lambda, \cdot ; v_{0}\right)$ is discrete holomorphic by Proposition 3.2, it is also discrete harmonic by Corollary 2.21. By this, we conclude that $\triangle G\left(v ; v_{0}\right)=0$.

It remains to show that there exists such a collection $C$ of loops with corresponding branches of log. We will show that a collection of sufficiently small counterclockwise oriented loops going once around each pole of $\mathrm{e}\left(\cdot, v^{\prime} ; v_{0}\right), v^{\prime}$ any vertex of the star of $v$, does the job, where around a pole $e$ of $\mathrm{e}\left(\cdot, v^{\prime} ; v_{0}\right)$ that branch of logarithm is taken where $\operatorname{Im}(\log (e)) \in\left(\arg \left(v^{\prime}-v_{0}\right)-\pi, \arg \left(v^{\prime}-v_{0}\right)+\pi\right)$. For this, we just have to show that the branches of the logarithm are well-defined. This is the case if for two vertices $v^{\prime}, v^{\prime \prime}$ of the star and a common pole $e$ of e $\left(\cdot, v^{\prime} ; v_{0}\right)$ and $\mathrm{e}\left(\cdot, v^{\prime \prime} ; v_{0}\right)$, there is an argument of $e$ contained in both $\left(\arg \left(v^{\prime}-v_{0}\right)-\pi, \arg \left(v^{\prime}-v_{0}\right)+\pi\right)$ and $\left(\arg \left(v^{\prime \prime}-v_{0}\right)-\pi, \arg \left(v^{\prime \prime}-v_{0}\right)+\pi\right)$.

It easily follows from $v \neq v_{0}$ that if $v^{\prime \prime}$ is not adjacent to $v^{\prime}$, there is a vertex $w$ adjacent to $v^{\prime}$ such that all common poles of $\mathrm{e}\left(\cdot, v^{\prime} ; v_{0}\right)$ and $\mathrm{e}\left(\cdot, v^{\prime \prime} ; v_{0}\right)$ are also common poles of $\mathrm{e}\left(\cdot, v^{\prime} ; v_{0}\right)$ and $\mathrm{e}\left(\cdot, w ; v_{0}\right)$. So let us assume without loss of generality that $v^{\prime}$ and $v^{\prime \prime}$ are adjacent. Clearly, we can also assume that both vertices are different from $v_{0}$ since $\mathrm{e}\left(\cdot, v_{0} ; v_{0}\right) \equiv 1$.

Let us suppose the converse from our claim, that means suppose that there is a common pole $e$ of $\mathrm{e}\left(\cdot, v^{\prime} ; v_{0}\right)$ and $\mathrm{e}\left(\cdot, v^{\prime \prime} ; v_{0}\right)$ such that no argument of the edge $e$ is contained in both the two intervals $\left(\arg \left(v^{\prime}-v_{0}\right)-\pi, \arg \left(v^{\prime}-v_{0}\right)+\pi\right)$ and $\left(\arg \left(v^{\prime \prime}-v_{0}\right)-\pi, \arg \left(v^{\prime \prime}-v_{0}\right)+\pi\right)$. This can only happen if the edge $v^{\prime} v^{\prime \prime}$ intersects the ray $v_{0}-t e, t \geqslant 0$. But since the edge $e$ is a pole of the discrete exponential, there is a strip with common parallel $e$, i.e., an infinite path in the dual graph $\diamond$ with edges dual to edges of $\Lambda$ that are parallel to $e$, that separates $v_{0}$ from both $v^{\prime}$ and $v^{\prime \prime}$ in such a way that $e$ is pointing toward the region of $v^{\prime}$ and $v^{\prime \prime}$ (see the first part of the appendix for more information on a strip). In particular, the edge $v^{\prime} v^{\prime \prime}$ is separated from the ray $v_{0}-t e, t \geqslant 0$, and cannot intersect it, contradiction.

Remark With almost the same arguments as in the proof of Proposition 3.4, we see that the discrete logarithm is a discrete holomorphic function on the vertices of $\tilde{\Lambda}_{v_{0}}$. In [2], it was shown that the discrete logarithm on rhombic quasicrystallic quad-graphs is even more than discrete holomorphic, namely isomonodromic.

Before we derive the asymptotics of the discrete Green's function given in Proposition 3.4, we state and prove some necessary estimations in two separate lemmas since we will use them later during the corresponding calculations for the discrete Cauchy's kernel in Sect. 3.4.

Lemma 3.5 Let $E_{1} \geqslant E_{0}>0$ be fixed real constants and consider a complex variable $\lambda$. Then, for any $e \in \mathbb{C} \backslash\{0\}$ satisfying $E_{1} \geqslant|e| \geqslant E_{0}$, the following holds true, where log denotes the principal branch of the logarithm and constants in $O$-notation depend on $E_{0}$ and $E_{1}$ only: 
(i) As $\lambda \rightarrow 0$,

$$
\begin{gathered}
-\frac{\lambda+e}{\lambda-e}=1+2 \frac{\lambda}{e}+2 \frac{\lambda^{2}}{e^{2}}+O\left(\lambda^{3}\right) \\
\log \left(-\frac{\lambda+e}{\lambda-e}\right)=2 \frac{\lambda}{e}+O\left(\lambda^{3}\right), \text { and } \log \left(-\frac{e}{\lambda-e}\right)=\frac{\lambda}{e}+O\left(\lambda^{2}\right) .
\end{gathered}
$$

(ii) As $|\lambda| \rightarrow \infty$,

$$
\begin{aligned}
\frac{\lambda+e}{\lambda-e} & =1+2 \frac{e}{\lambda}+2 \frac{e^{2}}{\lambda^{2}}+O\left(\lambda^{-3}\right) \\
\log \left(\frac{\lambda+e}{\lambda-e}\right) & =2 \frac{e}{\lambda}+O\left(\lambda^{-3}\right), \quad \text { and } \log \left(\frac{\lambda}{\lambda-e}\right)=\frac{e}{\lambda}+O\left(\lambda^{-2}\right) .
\end{aligned}
$$

Proof (i)

$$
-\frac{\lambda+e}{\lambda-e}=\frac{1+\frac{\lambda}{e}}{1-\frac{\lambda}{e}}=\left(1+\frac{\lambda}{e}\right)\left(1+\frac{\lambda}{e}+\frac{\lambda^{2}}{e^{2}}+O\left(\lambda^{3}\right)\right)=1+2 \frac{\lambda}{e}+2 \frac{\lambda^{2}}{e^{2}}+O\left(\lambda^{3}\right)
$$

shows the first equation and implies the second equation noting that

$$
\log (1+x)=x-x^{2} / 2+O\left(x^{3}\right) \text { as } x \rightarrow 0 .
$$

The series expansion for log also implies the third equation using

$$
\frac{-d}{\lambda-d}=\frac{1}{1-\frac{\lambda}{d}}=1+\frac{\lambda}{d}+O\left(\lambda^{2}\right)
$$

(ii) These equations are shown in a completely analogous way to (i), $e / \lambda$ taking the place of $\lambda / e$.

Lemma 3.6 Assume that there exist real constants $\alpha_{0}>0$ and $E_{1} \geqslant E_{0}>0$ such that $\alpha \geqslant \alpha_{0}$ and $E_{1} \geqslant e \geqslant E_{0}$ for all interior angles $\alpha$ and side lengths $e$ of parallelograms of $\Lambda$. Let $v_{0} \in V(\Lambda)$ and $Q_{0} \in V(\diamond)$ be fixed and consider $v \in V(\Lambda)$ and $Q \in V(\diamond)$ in the following.

(i) Let $k(v)$ be the combinatorial distance on $\Lambda$ between $v_{0}$ and $v$ (or between a vertex incident to $Q_{0}$ and $\left.v\right)$.

Then, $k(v)=\Omega\left(\left|v-v_{0}\right|\right)\left(\right.$ or $\left.k(v)=\Omega\left(\left|v-Q_{0}\right|\right)\right)$ as $|v| \rightarrow \infty$.

(ii) $J\left(v, v_{0}\right)=\Omega\left(v-v_{0}\right), J\left(Q, v_{0}\right)=\Omega\left(Q-v_{0}\right)$ and $J\left(Q, Q_{0}\right)=\Omega\left(Q-Q_{0}\right)$ as $|v|,|Q| \rightarrow \infty$.

(iii) $\tau\left(v, Q_{0}\right)=\Omega(1)$ and $\tau\left(Q, Q_{0}\right)=\Omega(1)$ as $|v|,|Q| \rightarrow \infty$.

(iv) Furthermore, assume that $\left|v-v_{0}\right| \geqslant 1$ and that the arguments of all oriented edges of a shortest directed path on $\Lambda$ from $v_{0}$ to $v$ can be chosen to lie in $\left[\theta_{0},-\theta_{0}\right]$, where $\theta_{0}:=-\left(\pi-\alpha_{0}\right) / 2$. 
Then, for any $\lambda \in\left[-E_{1} \sqrt{\left|v-v_{0}\right|},-E_{1} / \sqrt{\left|v-v_{0}\right|}\right]$ :

$$
\left|e\left(\lambda, v ; v_{0}\right)\right| \leqslant \exp \left(-\frac{\cos \left(\theta_{0}\right) \sqrt{\left|v-v_{0}\right|}}{2 E_{1}}\right) .
$$

Here, constants in the $\Omega$-notation depend on $\alpha_{0}, E_{0}$, and $E_{1}$ only.

Proof Let $e_{1}, e_{2}, \ldots, e_{k(v)}$ denote the oriented edges of $\Lambda$ of a shortest directed path on $\Lambda$ from $v_{0}$ (or a vertex incident to $Q_{0}$ ) to $v$. Due to the bound on the interior angles of parallelograms in $\Lambda$, there is a real $\theta_{0}$ such that the arguments of $e_{1}, e_{2}, \ldots, e_{k(v)}$ can be chosen to lie all in $\left[\theta_{0}, \theta_{0}+\pi-\alpha_{0}\right]$ by Lemma 4.2. All the claims in the first three parts stay (essentially) the same under rotation of the complex plane, so we may assume that $\theta_{0}=-\left(\pi-\alpha_{0}\right) / 2$. The same assumption is used in the fourth part.

(i) Under the assumptions above, the projections of the $e_{k}$ onto the real axis lie on the positive axis and are at least $E_{0} \cos \left(\theta_{0}\right)$ long since edge lengths are bounded by $E_{0}$ from below. It follows that $k(v) \leqslant \operatorname{Re}\left(v-v_{0}\right) /\left(E_{0} \cos \left(\theta_{0}\right)\right)$. Using in addition that $k(v) \geqslant\left|v-v_{0}\right| / E_{1}$, we get $k(v)=\Omega\left(\left|v-v_{0}\right|\right)$.

(ii) Using $1 /\left|E_{0}\right| \geqslant 1 /\left|e_{j}\right| \geqslant 1 /\left|E_{1}\right|$ for all $j$, we get

$$
\begin{aligned}
\left|J\left(v, v_{0}\right)\right| & =\left|\sum_{j=1}^{k(v)} e_{j}^{-1}\right| \leqslant \frac{k(v)}{E_{0}}=O\left(\left|v-v_{0}\right|\right), \\
\operatorname{Re}\left(J\left(v, v_{0}\right)\right) & =\operatorname{Re}\left(\sum_{j=1}^{k(v)} \frac{\bar{e}_{j}}{\left|e_{j}\right|^{2}}\right) \geqslant \frac{1}{E_{1}^{2}} \operatorname{Re}\left(\sum_{j=1}^{k(v)} e_{j}\right)=\frac{\cos \left(\theta_{0}\right)\left|v-v_{0}\right|}{E_{1}^{2}} .
\end{aligned}
$$

Hence, $J\left(v, v_{0}\right)=\Omega\left(\left|v-v_{0}\right|\right)$. This also implies that $J\left(Q, v_{0}\right)=\Omega\left(Q-v_{0}\right)$ since $\left|J\left(v, v_{0}\right)-J\left(Q, v_{0}\right)\right| \leqslant 1 /\left|E_{0}\right|$ for any $v$ incident to $Q$, which follows easily from the definition and the lower bound for edge lengths. Similarly, $J\left(Q, Q_{0}\right)=\Omega\left(Q-Q_{0}\right)$ follows from the previous statements if we take $v_{0}$ incident to $Q_{0}$.

(iii) $E_{0}^{-2} \geqslant\left|\tau\left(Q, Q_{0}\right)\right| \geqslant E_{1}^{-2}$ and $E_{0}^{-4} \geqslant\left|\tau\left(Q, Q_{0}\right)\right| \geqslant E_{1}^{-4}$ follow immediately from the definitions and the boundedness of edge lengths.

(iv) Using that $\lambda<0$ and $\operatorname{Re}(e)>0$, we get

$$
\frac{|\lambda+e|^{2}}{|\lambda-e|^{2}}=1+\frac{4 \lambda \operatorname{Re}(e)}{\lambda^{2}-2 \lambda \operatorname{Re}(e)+|e|^{2}} \leqslant 1+\frac{4 \lambda \operatorname{Re}(e)}{(\lambda-|e|)^{2}} \leqslant \exp \left(\frac{4 \lambda \operatorname{Re}(e)}{\left(\lambda-E_{1}\right)^{2}}\right) .
$$

Taking the product of such inequalities,

$$
\left|\mathrm{e}\left(\lambda, v ; v_{0}\right)\right| \leqslant \exp \left(\frac{2 \lambda \operatorname{Re}\left(v-v_{0}\right)}{\left(\lambda-E_{1}\right)^{2}}\right) \leqslant \exp \left(\frac{2 \lambda \cos \left(\theta_{0}\right)\left|v-v_{0}\right|}{\left(\lambda-E_{1}\right)^{2}}\right) .
$$

Now, we observe that $\lambda /\left(\lambda-E_{1}\right)^{2}$ attains its maximum on the boundary. Together with $\left|v-v_{0}\right| \geqslant 1$, 


$$
\frac{\lambda}{\left(\lambda-E_{1}\right)^{2}} \leqslant \frac{-\sqrt{\left|v-v_{0}\right|}}{E_{1}\left(1+\sqrt{\left|v-v_{0}\right|}\right)^{2}} \leqslant \frac{-1}{4 E_{1} \sqrt{\left|v-v_{0}\right|}}
$$

Plugging this into the estimation before gives the desired result.

Theorem 3.7 Assume that there exist real constants $\alpha_{0}>0$ and $E_{1} \geqslant E_{0}>0$ such that $\alpha \geqslant \alpha_{0}$ and $E_{1} \geqslant e \geqslant E_{0}$ for all interior angles $\alpha$ and side lengths $e$ of parallelograms of $\Lambda$. Let $v_{0} \in V(\Lambda)$ be fixed. Then, the following is true:

(i) The discrete Green's function $G\left(\cdot ; v_{0}\right)$ given in Proposition 3.4 has the following asymptotic behavior as $|v| \rightarrow \infty$ :

$$
\begin{aligned}
& G\left(v ; v_{0}\right)=\frac{1}{4 \pi} \log \left|\frac{v-v_{0}}{J\left(v, v_{0}\right)}\right|+O\left(\left|v-v_{0}\right|^{-2}\right) \text { if } v \text { and } v_{0} \text { are of different color, } \\
& G\left(v ; v_{0}\right)=\frac{\gamma_{\text {Euler }}+\log (2)}{2 \pi}+\frac{1}{4 \pi} \log \left|\left(v-v_{0}\right) J\left(v, v_{0}\right)\right|+O\left(\left|v-v_{0}\right|^{-2}\right) \text { otherwise. }
\end{aligned}
$$

(ii) There is exactly one discrete Green's function $G: V(\Lambda) \rightarrow \mathbb{R}$ for $v_{0}$ that behaves for $|v| \rightarrow \infty$ as

$$
\begin{aligned}
& G(v)=\frac{1}{4 \pi} \log \left|\frac{v-v_{0}}{J\left(v, v_{0}\right)}\right|+o\left(\left|v-v_{0}\right|^{-1 / 2}\right) \text { if } v \text { and } v_{0} \text { are of different color, } \\
& G(v)=\frac{\gamma_{\text {Euler }}+\log (2)}{2 \pi}+\frac{1}{4 \pi} \log \left|\left(v-v_{0}\right) J\left(v, v_{0}\right)\right|+o\left(\left|v-v_{0}\right|^{-1 / 2}\right) \text { otherwise. }
\end{aligned}
$$

Here, constants in the $O$-notation depend on $\alpha_{0}, E_{0}$, and $E_{1}$ only, and $\gamma_{\text {Euler }}$ denotes the Euler-Mascheroni constant.

Remark Note that due to Lemma 3.6(ii), $J\left(v, v_{0}\right)=\Omega\left(v-v_{0}\right)$ as $|v| \rightarrow \infty$.

By Proposition 4.3, we may replace the existence of constants $E_{1} \geqslant E_{0}>0$ such that $E_{1} \geqslant e \geqslant E_{0}$ for all side lengths $e$ of parallelograms by the existence of $q_{0}$ such that $e / e^{\prime} \geqslant q_{0}$ for the two side lengths $e, e^{\prime}$ of any parallelogram of $\Lambda$ since the latter implies the first assumption. Then, the constants in the $O$-notation depend instead of $E_{0}$ and $E_{1}$ on $q_{0}, e_{0}$, and $e_{1}$, where $e_{0}$ and $e_{1}$ are the side lengths of an arbitrary but fixed parallelogram of $\Lambda$.

The proof of the first part follows the ideas of Kenyon [16] and Bücking [5]. Both considered just quasicrystallic rhombic quad-graphs. But the main difference to [16] is that we deform the path of integration into an equivalent one different from Kenyon's, since his approach does not generalize to parallelogram-graphs. As Chelkak and Smirnov did for rhombic quad-graphs with bounded interior angles in [6], Kenyon used that two points $v, v^{\prime} \in V(\Lambda)$ can be connected by a directed path of edges such that the angle between each directed edge and $v^{\prime}-v$ is less than $\pi / 2$ or the angle between the sum of two consecutive edges and $v^{\prime}-v$ is less than $\pi / 2$. This is true for rhombic quad-graphs, but not for parallelogram-graphs. Instead, we use essentially the same deformation of the path of integration as Bücking did. 
Proof (i) The poles $e_{1}, \ldots, e_{k(v)}$ of $\mathrm{e}\left(\cdot, v ; v_{0}\right)$ correspond to the directed edges of a path from $v_{0}$ to $v$ of minimal length $k(v)$. By Lemma 4.2, there is a real $\theta_{0}$ such that the arguments of all directed edges above can be chosen to lie in $\left[\theta_{0}, \theta_{0}+\pi-\alpha_{0}\right]$. It is easy to check that the claim is invariant under rotation of the complex plane, so we can assume $\theta_{0}=-\left(\pi-\alpha_{0}\right) / 2$. By definition,

$$
G\left(v ; v_{0}\right)=\operatorname{Re}\left(\frac{1}{8 \pi^{2} i} \int_{C_{v}} \frac{\log \lambda}{\lambda} \mathrm{e}\left(\lambda, v ; v_{0}\right) d \lambda\right),
$$

where $C_{v}$ is a collection of sufficiently small counterclockwise oriented loops going once around each $e_{1}, \ldots, e_{k(v)}$ and $\log$ is the principal branch of the logarithm since it satisfies $\operatorname{Im}\left(\log \left(e_{j}\right)\right) \in\left[\theta_{0},-\theta_{0}\right]$ for all $j$.

By residue theorem, we can deform $C_{v}$ into a new path of integration $C_{v}^{\prime}$ that goes first along a circle centered at 0 with large radius $R(v)$ (such that all poles lie inside this disk) in counterclockwise direction starting and ending in $-R(v)$, then goes along the line segment $[-R(v),-r(v)]$ followed by the circle centered at 0 with small radius $r(v)$ (such that all poles lie outside this disk) in clockwise direction, and finally goes the line segment $[-R(v),-r(v)]$ backwards. Note that the principal branch of $\log$ jumps by $2 \pi i$ at the negative real axis where the integration along the two line segments takes place.

By assumption, $E_{0} \leqslant\left|e_{j}\right| \leqslant E_{1}$ for all $j$. Using $\left|v-v_{0}\right| \geqslant E_{0}$, it follows that

$$
\frac{E_{0}^{5}}{2}\left|v-v_{0}\right|^{-4} \leqslant \frac{E_{0}}{2}<\left|e_{j}\right|<2 E_{1} \leqslant 2 \frac{E_{1}}{E_{0}^{4}}\left|v-v_{0}\right|^{4} .
$$

In particular, we can choose

$$
R(v):=2 \frac{E_{1}}{E_{0}^{4}}\left|v-v_{0}\right|^{4} \text { and } r(v)=\frac{E_{0}^{5}}{2}\left|v-v_{0}\right|^{-4}
$$

for all $v \neq v_{0}$. We first look at the contributions of the circles with radii $r(v)$ and $R(v)$ to $G\left(v ; v_{0}\right)$.

Let $\lambda$ be on the small circle with radius $r(v)$. Then, $\lambda=\Omega\left(\left|v-v_{0}\right|^{-4}\right) \rightarrow 0$ as $|v| \rightarrow \infty$. In particular, we can apply $(-\lambda+e) /(\lambda-e)=1+2 \lambda / e+O\left(\lambda^{2}\right)$ by Lemma 3.5(i) to estimate $(-1)^{k(v)} \mathrm{e}\left(\lambda, v ; v_{0}\right)$. More precisely, the latter is a product of $k(v)=\Omega\left(\left|v-v_{0}\right|\right)$ terms (see Lemma 3.6(i)) with $e=e_{j}$. Multiplying out and using in addition $E_{0} \leqslant\left|e_{j}\right| \leqslant E_{1}$ easily gives for $|v| \rightarrow \infty$ that

$$
(-1)^{k(v)} \mathrm{e}\left(\lambda, v ; v_{0}\right)=1+O\left(\left|v-v_{0}\right|^{-3}\right) .
$$

Thus, we get for the integration along the small circle with radius $r(v)$ : 


$$
\begin{aligned}
& \operatorname{Re}\left(\frac{1}{8 \pi^{2} i} \int_{\pi}^{-\pi}(-1)^{k(v)} \frac{\log (r(v))+i \theta}{r(v) \exp (i \theta)}\left(1+O\left(\left|v-v_{0}\right|^{-3}\right)\right) d(r(v) \exp (i \theta))\right) \\
= & -\operatorname{Re}\left(\frac{1}{8 \pi^{2}} \int_{-\pi}^{\pi}(-1)^{k(v)}(\log (r(v))+i \theta)\left(1+O\left(\left|v-v_{0}\right|^{-3}\right)\right) d \theta\right) \\
= & -\frac{(-1)^{k(v)} \log (r(v))}{4 \pi}\left(1+O\left(\left|v-v_{0}\right|^{-3}\right)\right) .
\end{aligned}
$$

Let us now consider $\lambda$ to be on the large circle with radius $R(v)$. Then, we have $|\lambda|=\Omega\left(\left|v-v_{0}\right|^{4}\right) \rightarrow \infty$ as $|v| \rightarrow \infty$. Analogously to above, repeated use of the first equation in Lemma 3.5(ii) gives e $\left(\lambda, v ; v_{0}\right)=1+O\left(\left|v-v_{0}\right|^{-3}\right)$ as $|v| \rightarrow \infty$. Thus, $\log (R(v)) /(4 \pi) \cdot\left(1+O\left(\left|v-v_{0}\right|^{-3}\right)\right)$ is the contribution of the circle of radius $R(v)$. In total, the asymptotics for the real part of the integration along the two circles are

$$
\frac{1}{4 \pi}\left(\log (R(v))-(-1)^{k(v)} \log (r(v))\right)+O\left(\left|v-v_{0}\right|^{-2}\right) .
$$

The two integrations along $[-R(v),-r(v)]$ can be combined into the integral

$$
\frac{1}{4 \pi} \int_{-R(v)}^{-r(v)} \frac{\mathrm{e}\left(\lambda, v ; v_{0}\right)}{\lambda} d \lambda
$$

Since we are interested in the asymptotics for $|v| \rightarrow \infty$, we can consider $\left|v-v_{0}\right| \geqslant 1$ large enough and split the integration into the three parts along

$$
\left[-R(v),-E_{1} \sqrt{\left|v-v_{0}\right|}\right],\left[-E_{1} \sqrt{\left|v-v_{0}\right|},-\frac{E_{1}}{\sqrt{\left|v-v_{0}\right|}}\right], \text { and }\left[-\frac{E_{1}}{\sqrt{\left|v-v_{0}\right|}},-r(v)\right] .
$$

We first show that the contribution of $\lambda \in\left[-E_{1} \sqrt{\left|v-v_{0}\right|},-E_{1} / \sqrt{\left|v-v_{0}\right|}\right]$ can be neglected. Indeed, it is a consequence of the estimation in Lemma 3.6(iv) that

$$
\left|\frac{1}{4 \pi} \int_{-E_{1} \sqrt{\left|v-v_{0}\right|}}^{-E_{1} / \sqrt{\left|v-v_{0}\right|}} \frac{e\left(\lambda, v ; v_{0}\right)}{\lambda} d \lambda\right| \leqslant E_{1} \sqrt{\left|v-v_{0}\right|} \exp \left(-\frac{\cos \left(\theta_{0}\right) \sqrt{\left|v-v_{0}\right|}}{2 E_{1}}\right) .
$$

As $|v| \rightarrow \infty$, the latter expression decays faster to zero than any power of $\left|v-v_{0}\right|$.

Now, consider $\lambda \in\left[-E_{1} / \sqrt{\left|v-v_{0}\right|},-r(v)\right]$. Then, $\lambda \rightarrow 0$ as $|v| \rightarrow \infty$, so using the second equation in Lemma 3.5(i) $k(v)=\Omega\left(\left|v-v_{0}\right|\right)$ times gives as $|v| \rightarrow \infty$ :

$$
\begin{aligned}
(-1)^{k(v)} \mathrm{e}\left(\lambda, v ; v_{0}\right) & =\exp \left(2 \lambda J\left(v, v_{0}\right)+O\left(k(v) \lambda^{3}\right)\right) \\
& =\exp \left(2 \lambda J\left(v, v_{0}\right)\right)\left(1+O\left(\left|v-v_{0}\right| \lambda^{3}\right)\right) .
\end{aligned}
$$


Thus, the integral near the origin is equal to

$$
\frac{(-1)^{k(v)}}{4 \pi} \int_{-E_{1} / \sqrt{\left|v-v_{0}\right|}}^{-r(v)}\left(\frac{\exp \left(2 \lambda J\left(v, v_{0}\right)\right)}{\lambda}+\exp \left(2 \lambda J\left(v, v_{0}\right)\right) O\left(\left|v-v_{0}\right| \lambda^{2}\right)\right) d \lambda .
$$

The expansion of the integral of the second term involves two exponential factors, one for each bound: $\exp \left(-2 J\left(v, v_{0}\right) r(v)\right)$ and $\exp \left(-2 E_{1} J\left(v, v_{0}\right) / \sqrt{\left|v-v_{0}\right|}\right)$. Now, we will use that $J\left(v, v_{0}\right)=\Omega\left(\left|v-v_{0}\right|\right)$ by Lemma 3.6(ii). Since the exponent of the first factor goes to 0 in speed $\left|v-v_{0}\right|^{-3}$, the exponential goes exponentially fast to 1 as $|v| \rightarrow \infty$. For the second factor, we use our assumption that the arguments of all the poles can be chosen to lie in $\left[-\left(\pi-\alpha_{0}\right) / 2,\left(\pi-\alpha_{0}\right) / 2\right]$. It follows that $\operatorname{Re}\left(J\left(v, v_{0}\right)\right)$ is positive and goes to infinity like $\Omega\left(\left|v-v_{0}\right|\right)$ as $|v| \rightarrow \infty$, such that the second exponential factor goes to zero exponentially fast. Now, it is not hard to see that the integral of $\exp \left(2 \lambda J\left(v, v_{0}\right)\right) O\left(\left|v-v_{0}\right| \lambda^{3}\right)$ gives $O\left(\left|v-v_{0}\right|^{-2}\right)$. For the first term, we get

$$
\begin{aligned}
& \frac{(-1)^{k(v)}}{4 \pi}\left(\int_{-2 E_{1} J\left(v, v_{0}\right) / \sqrt{\left|v-v_{0}\right|}}^{-1} \frac{\exp (s)}{s} d s+\int_{-1}^{-2 r(v) J\left(v, v_{0}\right)} \frac{\exp (s)-1}{s} d s\right) \\
+ & \frac{(-1)^{k(v)}}{4 \pi} \int_{-1}^{-2 r(v) J\left(v, v_{0}\right)} \frac{1}{s} d s \\
= & \frac{(-1)^{k(v)}}{4 \pi}\left(\int_{-\infty}^{-1} \frac{\exp (s)}{s} d s+\int_{-1}^{0} \frac{\exp (s)-1}{s} d s\right)+\frac{(-1)^{k(v)}}{4 \pi} \log \left(2 r(v) J\left(v, v_{0}\right)\right) \\
- & \frac{(-1)^{k(v)}}{4 \pi}\left(\int_{-\infty}^{-2 E_{1} J\left(v, v_{0}\right) / \sqrt{\left|v-v_{0}\right|}} \int_{-2 r(v) J\left(v, v_{0}\right)} \frac{\exp (s)}{s} d s+s\right)-1 \\
= & \frac{(-1)^{k(v)}}{4 \pi}\left(\gamma_{\text {Euler }}+\Omega\left(\left|v-v_{0}\right|^{-3}\right)+\log \left(2 r(v) J\left(v, v_{0}\right)\right)\right) .
\end{aligned}
$$

To get to the last line, we used that $\operatorname{Re}\left(J\left(v, v_{0}\right)\right)=\Omega\left(\left|v-v_{0}\right|\right)$ as $|v| \rightarrow \infty$ stays positive. Indeed, as $|v| \rightarrow \infty$, the first integral in the second to last line goes to zero exponentially fast (to see this, just write the integrand as $s \exp (s) / s^{2}$ and bound the absolute value of the integral from above by $s_{0} \exp \left(s_{0}\right)$ where $s_{0}$ denotes the term $\left.-2 E_{1} J\left(v, v_{0}\right) / \sqrt{\left|v-v_{0}\right|}\right)$ and the second integral is of order $\Omega\left(\left|v-v_{0}\right|^{-3}\right)$ as $|v| \rightarrow \infty$ as a Taylor expansion of the exponential shows.

Finally, let $\lambda \in\left[-R(v),-E_{1} \sqrt{\left|v-v_{0}\right|}\right]$. Then, $\lambda \rightarrow-\infty$ as $|v| \rightarrow \infty$, and repeated use of the second equation in Lemma 3.5(ii) gives 


$$
\mathrm{e}\left(\lambda, v ; v_{0}\right)=\exp \left(\frac{2\left(v-v_{0}\right)}{\lambda}\right)\left(1+O\left(\left|v-v_{0}\right| \lambda^{-3}\right)\right)
$$

as $|v| \rightarrow \infty$, so the corresponding contribution of the integral to $4 \pi G\left(v ; v_{0}\right)$ is

$$
\begin{aligned}
& \int_{-R(v)}^{-E_{1} \sqrt{\left|v-v_{0}\right|}} \frac{\exp \left(\lambda, v ; v_{0}\right)}{\lambda} d \lambda=\int_{-R(v)}^{-E_{1} \sqrt{\left|v-v_{0}\right|}} \frac{\exp \left(\frac{2\left(v-v_{0}\right)}{\lambda}\right)}{\lambda} d \lambda+O\left(\left|v-v_{0}\right|^{-2}\right) \\
= & \int_{-R(v) /\left(2\left(v-v_{0}\right)\right)}^{-1} \frac{\exp \left(\frac{1}{s}\right)-1}{s} d s+\int_{-1}^{-E_{1} \sqrt{\mid v-v_{0} / /\left(2\left(v-v_{0}\right)\right)}} \frac{\exp \left(\frac{1}{s}\right)}{s} d s \\
+ & \int_{-R(v) /\left(2\left(v-v_{0}\right)\right)}^{-1} \frac{1}{s} d s+O\left(\left|v-v_{0}\right|^{-2}\right) \\
= & \gamma_{\text {Euler }}-\log \left(\frac{R(v)}{2\left(v-v_{0}\right)}\right)+O\left(\left|v-v_{0}\right|^{-2}\right)
\end{aligned}
$$

by a similar reasoning as above. Summing up the integrals over all four parts of the contour and taking the real part, we finally get that $4 \pi G\left(v ; v_{0}\right)$ equals

$$
\left(1+(-1)^{k(v)}\right)\left(\gamma_{\text {Euler }}+\log (2)\right)+\log \left|v-v_{0}\right|+(-1)^{k(v)} \log \left|J\left(v, v_{0}\right)\right|+O\left(\left|v-v_{0}\right|^{-2}\right) .
$$

(ii) We know from Theorem 2.31 that discrete harmonic functions of asymptotics $o\left(\left|v-v_{0}\right|^{-1 / 2}\right)$ as $|v| \rightarrow \infty$ are zero. We can apply this result to the discrete harmonic function $G-G\left(\cdot ; v_{0}\right)$, where $G\left(\cdot ; v_{0}\right)$ from the first part has the desired asymptotics.

Remark Let us compare this result to the case of rhombi of side length one. Assume that $v_{0} \in V(\Gamma)$. Then, the discrete logarithm is purely real and nonbranched on $V(\Gamma)$ and purely imaginary and branched on $V\left(\Gamma^{*}\right)$. It follows that $G\left(v ; v_{0}\right)=0$ if $v \in V\left(\Gamma^{*}\right)$, well fitting to the fact that $\triangle$ splits into two discrete Laplacians on $\Gamma$ and $\Gamma^{*}$. Using $J\left(v, v_{0}\right)=\overline{v-v_{0}}$,

$$
G\left(v ; v_{0}\right)=\frac{1}{2 \pi}\left(\gamma_{\text {Euler }}+\log (2)+\log \left|v-v_{0}\right|\right)+O\left(\left|v-v_{0}\right|^{-2}\right)
$$

as $|v| \rightarrow \infty$ for $v \in V(\Gamma)$, exactly as in the work of Bücking [5], who slightly strengthened the error term in Kenyon's work [16]. In this paper, Kenyon showed that there is no further discrete Green's function with asymptotics $o\left(\left|v-v_{0}\right|\right)$. 


\subsection{Asymptotics of Discrete Cauchy's Kernels}

Let $v_{0} \in V(\Lambda)$ and $Q_{0} \in V(\diamond)$ be given. We first give a formula for a discrete Cauchy's kernel $K_{v_{0}}$ with respect to $v_{0}$ on $V(\diamond)$ that has asymptotics $\Omega\left(\left|Q-v_{0}\right|^{-1}\right)$ as $|Q| \rightarrow \infty$. Remember that the vertex $Q \in V(\diamond) \cong F(\Lambda)$ is placed on the center of the corresponding parallelogram. Then, we provide a discrete Cauchy's kernel $K_{Q_{0}}$ with respect to $Q_{0}$ on $V(\Lambda)$ with asymptotics $\Omega\left(\left|v-Q_{0}\right|^{-1}\right)$ as $v \rightarrow \infty$. In both cases, there are no further discrete Cauchy's kernels with asymptotics $o\left(\left|Q-v_{0}\right|^{-1 / 2}\right)$ or $o\left(\left|v-Q_{0}\right|^{-1 / 2}\right)$ as $|Q|,|v| \rightarrow \infty$. In the end of this section, the asymptotics of $\partial_{\Lambda} K_{Q_{0}}$ are determined.

Theorem 3.8 Let $G\left(\cdot ; v_{0}\right)$ be a discrete Green's function on $V(\diamond)$ for $v_{0} \in V(\Lambda)$.

(i) $K_{v_{0}}:=8 \pi \partial_{\Lambda} G\left(\cdot ; v_{0}\right)$ is a discrete Cauchy's kernel with respect to $v_{0}$.

(ii) Assume additionally that there exist real constants $\alpha_{0}>0$ and $E_{1} \geqslant E_{0}>0$ such that $\alpha \geqslant \alpha_{0}$ and $E_{1} \geqslant e \geqslant E_{0}$ for all interior angles $\alpha$ and side lengths $e$ of parallelograms of $\Lambda$. Suppose that $G\left(\cdot ; v_{0}\right)$ is the discrete Green's function given in Proposition 3.4 and $K_{v_{0}}$ the discrete Cauchy's kernel given in (i). Then,

$$
K_{v_{0}}(Q)=\frac{1}{Q-v_{0}}+\frac{\tau\left(Q, v_{0}\right)}{J\left(Q, v_{0}\right)}+O\left(\left|Q-v_{0}\right|^{-2}\right)
$$

as $|Q| \rightarrow \infty$. Here, constants in the $O$-notation depend on $\alpha_{0}, E_{0}$, and $E_{1}$ only.

(iii) Under the conditions of (ii), there is exactly one discrete Cauchy's kernel with respect to $v_{0}$ with asymptotics $o\left(\left|Q-v_{0}\right|^{-1 / 2}\right)$ as $|Q| \rightarrow \infty$.

Remark Note that due to Lemma 3.6(ii) and (iii),

$$
\frac{\tau\left(Q, v_{0}\right)}{J\left(Q, v_{0}\right)}=\Omega\left(\left(Q-v_{0}\right)^{-1}\right)
$$

as $|Q| \rightarrow \infty$. As in Theorem 3.7, we may replace the existence of $E_{1} \geqslant E_{0}>0$ such that $E_{1} \geqslant e \geqslant E_{0}$ for all side lengths $e$ of parallelograms by the existence of $q_{0}$ such that $e / e^{\prime} \geqslant q_{0}$ for the two side lengths $e, e^{\prime}$ of any parallelogram of $\Lambda$ since the latter implies the first assumption by Proposition 4.3. Then, the constants in the $O$-notation depend instead of $E_{0}$ and $E_{1}$ on $q_{0}, e_{0}$, and $e_{1}$, where $e_{0}$ and $e_{1}$ are the side lengths of an arbitrary but fixed parallelogram of $\Lambda$.

Proof (i) By Corollary 2.20 about the factorization of the discrete Laplacian,

$$
\bar{\partial}_{\diamond} K_{v_{0}}(v)=8 \pi\left(\bar{\partial}_{\diamond} \partial_{\Lambda} G\left(\cdot ; v_{0}\right)\right)(v)=2 \pi \Delta G\left(v ; v_{0}\right)=\delta_{v v_{0}} \frac{\pi}{\operatorname{ar}\left(F_{v_{0}}\right)} .
$$

(ii) For the ease of notation, we assume that $v_{0} \in V(\Gamma)$, but note that the other case of a white vertex $v_{0}$ can be handled in the same manner. Let $b_{-}, w_{-}, b_{+}, w_{+}$ denote the vertices of the parallelogram $Q$ in counterclockwise order, starting with a black vertex. Using the asymptotics of Theorem 3.7, as $|Q| \rightarrow \infty$ : 


$$
\begin{aligned}
& 4 \pi G\left(b_{+} ; v_{0}\right)-4 \pi G\left(b_{-} ; v_{0}\right)=\log \left(\frac{\left|J\left(b_{+}, v_{0}\right)\right|\left|b_{+}-v_{0}\right|}{\left|J\left(b_{-}, v_{0}\right)\right|\left|b_{-}-v_{0}\right|}\right)+O\left(\left|Q-v_{0}\right|^{-2}\right), \\
& 4 \pi G\left(w_{+} ; v_{0}\right)-4 \pi G\left(w_{-} ; v_{0}\right)=\log \left(\frac{\left|J\left(w_{-}, v_{0}\right)\right|\left|w_{+}-v_{0}\right|}{\left|J\left(w_{+}, v_{0}\right)\right|\left|w_{-}-v_{0}\right|}\right)+O\left(\left|Q-v_{0}\right|^{-2}\right) .
\end{aligned}
$$

Let $a:=w_{+}-b_{-}$and $b:=w_{-}-b_{-}$. Using $\log |1+x|=(x+\bar{x}) / 2+O\left(x^{2}\right)$ as $x \rightarrow 0$ and $J\left(Q, v_{0}\right)=\Omega\left(\left|Q-v_{0}\right|\right)$ as $|Q| \rightarrow \infty$ given by Lemma 3.6(ii), we get as $|Q| \rightarrow \infty$ :

$$
\begin{aligned}
\log \left|b_{ \pm}-v_{0}\right| & =\log \left|\left(Q-v_{0}\right)\left(1 \pm \frac{a+b}{2\left(Q-v_{0}\right)}\right)\right| \\
& =\log \left|Q-v_{0}\right| \pm \operatorname{Re}\left(\frac{a+b}{2\left(Q-v_{0}\right)}\right)+O\left(\left|Q-v_{0}\right|^{-2}\right) \\
\log \left|w_{ \pm}-v_{0}\right| & =\log \left|Q-v_{0}\right| \pm \operatorname{Re}\left(\frac{a-b}{2\left(Q-v_{0}\right)}\right)+O\left(\left|Q-v_{0}\right|^{-2}\right) \\
\log \left|J\left(b_{ \pm}, v_{0}\right)\right| & =\log \left|J\left(Q, v_{0}\right)\left(1 \pm \frac{a^{-1}+b^{-1}}{2 J\left(Q, v_{0}\right)}\right)\right| \\
& =\log \left|J\left(Q, v_{0}\right)\right| \pm \operatorname{Re}\left(\frac{a^{-1}+b^{-1}}{2 J\left(Q, v_{0}\right)}\right)+O\left(\left|Q-v_{0}\right|^{-2}\right) \\
\log \left|J\left(w_{ \pm}, v_{0}\right)\right| & =\log \left|J\left(Q, v_{0}\right)\right| \pm \operatorname{Re}\left(\frac{a^{-1}-b^{-1}}{2 J\left(Q, v_{0}\right)}\right)+O\left(\left|Q-v_{0}\right|^{-2}\right)
\end{aligned}
$$

Therefore, we get for the discrete derivative of $8 \pi G\left(\cdot ; v_{0}\right)$ at the face $Q$ :

$$
\begin{aligned}
K_{v_{0}}(Q) & =\lambda Q \frac{\operatorname{Re}\left(\frac{a+b}{Q-v_{0}}\right)+\operatorname{Re}\left(\frac{a^{-1}+b^{-1}}{J\left(Q, v_{0}\right)}\right)}{a+b}+\bar{\lambda}_{Q} \frac{\operatorname{Re}\left(\frac{a-b}{Q-v_{0}}\right)-\operatorname{Re}\left(\frac{a^{-1}-b^{-1}}{J\left(Q, v_{0}\right)}\right)}{a-b} \\
& +O\left(\left|Q-v_{0}\right|^{-2}\right) \\
& =\frac{1}{Q-v_{0}}+\frac{1}{a b J\left(Q, v_{0}\right)}+O\left(\left|Q-v_{0}\right|^{-2}\right) \\
& +\frac{1}{2}\left(\lambda \frac{\overline{a+b}}{a+\bar{\lambda}} \frac{\overline{a-b}}{a-b}\right)\left(\frac{1}{\overline{Q-v_{0}}}+\frac{1}{\overline{a b J\left(Q, v_{0}\right)}}\right) \\
& =\frac{1}{Q-v_{0}}+\frac{\tau\left(Q, v_{0}\right)}{J\left(Q, v_{0}\right)}+O\left(\left|Q-v_{0}\right|^{-2}\right)
\end{aligned}
$$

as $|Q| \rightarrow \infty$. Here, $\lambda_{Q}=\exp \left(-i\left(\varphi_{Q}-\pi / 2\right)\right)$, and we have used the identity

$$
-\frac{\lambda_{Q}}{\bar{\lambda}_{Q}}=\exp \left(-2 i \varphi_{Q}\right)=\frac{a+b}{a-b} \cdot \frac{\overline{a-b}}{\overline{a+b}} \Leftrightarrow \lambda_{Q} \frac{\overline{a+b}}{a+b}+\bar{\lambda}_{Q} \frac{\overline{a-b}}{a-b}=0 .
$$


(iii) Let $h$ be the difference of two discrete Cauchy's kernels with respect to $v_{0}$ with asymptotics $o\left(\left|Q-v_{0}\right|^{-1 / 2}\right)$ as $|Q| \rightarrow \infty . K_{v_{0}}$ in the second part is such a discrete Cauchy's kernel. Then, $h$ is discrete holomorphic, and by Proposition 2.8, a discrete primitive $f$ on $V(\Lambda)$ exists. By construction,

$$
\frac{f\left(b_{+}\right)-f\left(b_{-}\right)}{b_{+}-b_{-}}=\frac{f\left(w_{+}\right)-f\left(w_{-}\right)}{w_{+}-w_{-}}=\partial_{\Lambda} f(Q)=h(Q)=o\left(\left|Q-v_{0}\right|^{-1 / 2}\right) \text {. }
$$

Since angles and edge lengths of parallelograms are bounded, the conditions of Theorem 2.31 are fulfilled, implying that $f$ is biconstant, so $h$ vanishes identically.

Since we do not have discrete Green's functions on $V(\diamond)$, we have to derive a discrete Cauchy's kernels on $V(\Lambda)$ differently. To do so, we follow the original approach of Kenyon using the discrete exponential [16] that was reintroduced by Chelkak and Smirnov in [6].

Proposition 3.9 Let $Q_{0} \in V(\diamond)$. The function defined by

$$
K_{Q_{0}}(v):=\frac{1}{\pi i} \int_{C_{v}} \log (\lambda) e\left(\lambda, v ; Q_{0}\right) d \lambda=2 \int_{-\left(v-Q_{0}\right) \infty}^{0} e\left(\lambda, v ; Q_{0}\right) d \lambda
$$

is a discrete Cauchy's kernel with respect to $Q_{0}$. Here, $C_{v}$ is a collection of sufficiently small counterclockwise oriented loops going once around each pole of $e\left(\cdot, v ; Q_{0}\right)$. On each loop around a pole e of $e\left(\cdot, v ; Q_{0}\right)$, we take the branch of logarithm that satisfies $\operatorname{Im}(\log (e)) \in\left(\theta_{v}-\pi, \theta_{v}+\pi\right)$, where $\theta_{v}:=\arg \left(v-Q_{0}\right)$. The second integration goes along the straight ray that ends in the origin in direction $\left(v-Q_{0}\right)$.

Proof Lemma 4.2 assures that the arguments of all poles can be indeed chosen in such a way that they lie in $\left(\theta_{v}-\pi, \theta_{v}+\pi\right)$.

By residue theorem, we can replace integration along $C_{v}$ by an integration along a circle centered at 0 with large radius $R$ (such that all poles lie inside the disk) in counterclockwise direction, an integration along a circle centered at 0 with small radius $r$ (such that all poles lie outside the disk) in clockwise direction, and integrations along the two directions of the line segment $[-R,-r]$. Using that

$$
\lim _{|\lambda| \rightarrow \infty} \lambda \mathrm{e}\left(\lambda, v ; Q_{0}\right) \log (\lambda)=0
$$

and taking $r$ arbitrarily small, there is a homotopy between the new integration path and the path from complex infinity to 0 and back along the ray spanned by $-\left(v-Q_{0}\right)$ that does not change the value of the integral. The branch of log we consider jumps by $2 \pi i$ on the two sides of the ray, which shows

$$
\frac{1}{\pi i} \int_{C_{v}} \log (\lambda) \mathrm{e}\left(\lambda, v ; Q_{0}\right) d \lambda=2 \int_{-\left(v-Q_{0}\right) \infty}^{0} \mathrm{e}\left(\lambda, v ; Q_{0}\right) d \lambda
$$


In particular, $\theta_{v}$ can be replaced by any $\theta_{v}+2 k \pi, k \in \mathbb{Z}$, in the first integration.

Let us consider the parallelogram $Q_{0}$ with vertices $b_{-}, w_{-}, b_{+}, w_{+}$in counterclockwise direction, starting with a black vertex, and let us denote $a:=w_{+}-b_{-}$, $b:=w_{-}-b_{-}$. Without loss of generality, we rotate the complex plane in such a way that $\arg \left(b_{+}-Q_{0}\right)=0$. Then, $\arg (a)$ and $\arg (b)$ lie between 0 and $\pm \pi$, and using residue theorem,

$$
\begin{aligned}
& K_{Q_{0}}\left(b_{-}\right)=2 \frac{(\log |a|+i \arg (a)+i \pi)-(\log |b|+i \arg (b)+i \pi)}{b-a} \\
& K_{Q_{0}}\left(b_{+}\right)=2 \frac{(\log |b|+i \arg (b))-(\log |a|+i \arg (a))}{b-a} \\
& K_{Q_{0}}\left(w_{-}\right)=2 \frac{(\log |b|+i \arg (b)+2 i \pi)-(\log |a|+i \arg (a)+i \pi)}{a+b} \\
& K_{Q_{0}}\left(w_{+}\right)=2 \frac{(\log |a|+i \arg (a))-(\log |b|+i \arg (b)+i \pi)}{a+b}
\end{aligned}
$$

Finally, we get by Lemma 2.3 giving an integration formula for $\bar{\partial}_{\Lambda}$ :

$$
\begin{aligned}
\bar{\partial}_{\Lambda} K_{Q_{0}}\left(Q_{0}\right) & =\frac{(a-b)\left(K_{Q_{0}}\left(b_{+}\right)-K_{Q_{0}}\left(b_{+}\right)\right)+(a+b)\left(K_{Q_{0}}\left(w_{-}\right)-K_{Q_{0}}\left(w_{+}\right)\right)}{4 i \operatorname{ar}\left(F_{Q_{0}}\right)} \\
& =\frac{\pi}{\operatorname{ar}\left(F_{Q_{0}}\right)} .
\end{aligned}
$$

By a similar argument as in the proof of Proposition 3.4, we can choose the same contours of integration for all incident vertices $v$ of a face $Q \neq Q_{0}$. Then, the discrete derivative $\bar{\partial}_{\Lambda}$ commutes with the integration, and $\bar{\partial}_{\Lambda} K_{Q_{0}}(Q)=0$ because $\left(\lambda, \cdot ; Q_{0}\right)$ is discrete holomorphic by Proposition 3.2.

Theorem 3.10 Assume that there are $\alpha_{0}>0$ and $E_{1} \geqslant E_{0}>0$ such that $\alpha \geqslant \alpha_{0}$ and $E_{1} \geqslant e \geqslant E_{0}$ for all interior angles $\alpha$ and side lengths $e$ of parallelograms of $\Lambda$. Let $Q_{0} \in V(\diamond)$ be fixed.

(i) The discrete Cauchy's kernel $K_{Q_{0}}$ given in Proposition 3.9 has the following asymptotics as $|v| \rightarrow \infty$ :

$$
K_{Q_{0}}(v)=\frac{1}{v-Q_{0}}+\frac{\tau\left(v, Q_{0}\right)}{J\left(v, Q_{0}\right)}+O\left(\left|v-Q_{0}\right|^{-3}\right) .
$$

(ii) There is no further discrete Cauchy's kernel with respect to $Q_{0}$ that has asymptotics $o\left(\left|v-Q_{0}\right|^{-1 / 2}\right)$ as $|v| \rightarrow \infty$.

(iii) For the discrete Cauchy's kernel $K_{Q_{0}}$ given in Proposition 3.9, $\partial_{\Lambda} K_{Q_{0}}$ has the following asymptotics as $|v| \rightarrow \infty$ :

$$
\partial_{\Lambda} K_{Q_{0}}(Q)=-\frac{1}{\left(Q-Q_{0}\right)^{2}}-\frac{\tau\left(Q, Q_{0}\right)}{J\left(Q, Q_{0}\right)^{2}}+O\left(\left|Q-Q_{0}\right|^{-3}\right) .
$$


(iv) Up to two additive complex constants on $\Gamma$ and $\Gamma^{*}$, there is no further discrete Cauchy's kernel with respect to $Q_{0}$ such that its discrete derivative has asymptotics $o\left(\left|Q-Q_{0}\right|^{-1 / 2}\right)$ as $|Q| \rightarrow \infty$.

Here, constants in the $O$-notation depend on $\alpha_{0}, E_{0}$, and $E_{1}$ only.

Remark By Lemma 3.6(ii) and (iii),

$$
\frac{\tau\left(v, Q_{0}\right)}{J\left(v, Q_{0}\right)}=\Omega\left(\left(v-Q_{0}\right)^{-1}\right) \quad \text { and } \quad \frac{\tau\left(Q, Q_{0}\right)}{J\left(Q, Q_{0}\right)^{2}}=\Omega\left(\left(Q-Q_{0}\right)^{-2}\right)
$$

as $|v|,|Q| \rightarrow \infty$. As in the previous Theorem 3.8, we may replace the existence of constants $E_{1} \geqslant E_{0}>0$ such that $E_{1} \geqslant e \geqslant E_{0}$ for all side lengths $e$ of parallelograms by the existence of $q_{0}$ such that $e / e^{\prime} \geqslant q_{0}$ for the two side lengths $e, e^{\prime}$ of any parallelogram of $\Lambda$ since the latter implies the first assumption by Proposition 4.3. Then, the constants in the $O$-notation depend instead of $E_{0}$ and $E_{1}$ on $q_{0}, e_{0}$, and $e_{1}$, where $e_{0}$ and $e_{1}$ are the side lengths of an arbitrary but fixed parallelogram of $\Lambda$.

The proof of the first part follows the ideas of Kenyon [16]. Similar to the proof of Theorem 3.7, the path of integration is deformed into a path different from the one Kenyon used, $\left(-\left(v-Q_{0}\right) \infty, 0\right]$. For the same reasons as before, his approach does not generalize to parallelogram-graphs. The second and the fourth part of the theorem are immediate consequences of Theorem 2.31; the third part is shown by a direct computation.

Proof (i) Among all the vertices incident to $Q_{0}$, let $v_{0}$ be the one that is combinatorially closest to $v$ on $\Lambda$. Then, the poles $d_{1}, d_{2}, e_{1}, \ldots, e_{k(v)}$ of e $\left(\cdot, v ; Q_{0}\right)$ correspond to the directed edges of a shortest path from $v_{0}$ to $v$ of length $k(v)$ and the two directed edges of $Q_{0}$ that point toward $v_{0}$. It is easy to deduce from Lemma 4.2 that the arguments of all poles can be chosen to lie in $\left[\theta_{0}, \theta_{0}+\pi-\alpha_{0}\right]$. For more details, we refer to the appendix in [13]. The claim is invariant under multiplication of the complex plane, so we can assume that $\theta_{0}=-\left(\pi-\alpha_{0}\right) / 2$. Then, there are no poles in the left half-plane, such that we can reduce the calculation to an integration over $\mathbb{R}$ :

$$
K_{Q_{0}}(v)=2 \int_{-\left(v-Q_{0}\right) \infty}^{0} \mathrm{e}\left(\lambda, v ; Q_{0}\right) d \lambda=2 \int_{-\infty}^{0} \mathrm{e}\left(\lambda, v ; Q_{0}\right) d \lambda
$$

Since we are interested in the limit $|v| \rightarrow \infty$, we take $\left|v-Q_{0}\right| \geqslant 1$ large enough and split the integration into the three parts along the intervals $\left(-\infty,-E_{1} \sqrt{\left|v-Q_{0}\right|}\right]$, $\left[-E_{1} \sqrt{\left|v-Q_{0}\right|},-E_{1} / \sqrt{\left|v-Q_{0}\right|}\right],\left[-E_{1} / \sqrt{\left|v-Q_{0}\right|}, 0\right]$. Lemma 3.6(iv) shows that the contribution of the range $\lambda \in\left[-E_{1} \sqrt{\left|v-Q_{0}\right|},-E_{1} / \sqrt{\left|v-Q_{0}\right|}\right]$ decays faster to zero than any power of $v-Q_{0}$ as $|v| \rightarrow \infty$. By Lemma 3.6(i) and (ii) we know that $k(v)=\Omega\left(v-Q_{0}\right)$ and $J\left(v, Q_{0}\right)=\Omega\left(v-Q_{0}\right)$ as $|v| \rightarrow \infty$. Since the arguments of all the poles can be chosen to lie in $\left[-\left(\pi-\alpha_{0}\right) / 2,\left(\pi-\alpha_{0}\right) / 2\right]$, it follows even that $\operatorname{Re}\left(J\left(v, Q_{0}\right)\right)=\Omega\left(\left|v-Q_{0}\right|\right)$ as $|v| \rightarrow \infty$ stays positive. 
Let $\lambda \in\left[-E_{1} / \sqrt{\left|v-Q_{0}\right|}, 0\right]$. Then, $\lambda \rightarrow 0$ as $|v| \rightarrow \infty$. Repeated use of the second and the third equation in Lemma 3.5(i) yields

$$
\mathrm{e}\left(\lambda, v ; Q_{0}\right)=\tau\left(v, Q_{0}\right) \exp \left(2 \lambda J\left(v, Q_{0}\right)\right)\left(1+O\left(\lambda^{2}\right)+O\left(\left|v-Q_{0}\right| \lambda^{3}\right)\right)
$$

as $|v| \rightarrow \infty$. Thus, the integral near the origin behaves for $|v| \rightarrow \infty$ as

$$
2 \int_{-E_{1} / \sqrt{\left|v-Q_{0}\right|}}^{0} \mathrm{e}\left(\lambda, v ; Q_{0}\right) d \lambda=\frac{\tau\left(v, Q_{0}\right)}{J\left(v, Q_{0}\right)}+O\left(\left|v-Q_{0}\right|^{-3}\right)
$$

For this, we used that $\exp \left(-2 E_{1} J\left(v, Q_{0}\right) / \sqrt{\left|v-Q_{0}\right|}\right)$ goes to zero exponentially fast as $|v| \rightarrow \infty$.

Now, let $\lambda \in\left(-\infty,-E_{1} \sqrt{\left|v-Q_{0}\right|}\right]$. Then, $\lambda \rightarrow-\infty$ as $|v| \rightarrow \infty$. Repeated use of the second and the third equation in Lemma 3.5(ii) shows that

$$
\mathrm{e}\left(\lambda, v ; Q_{0}\right)=\lambda^{-2} \exp \left(2\left(v-Q_{0}\right) / \lambda\right)\left(1+O\left(\lambda^{-2}\right)+O\left(\left|v-Q_{0}\right| \lambda^{-3}\right)\right)
$$

as $|v| \rightarrow \infty$. Using the result of above, we get for the integral near minus infinity:

$$
\begin{aligned}
& 2 \int_{-\infty}^{-E_{1} \sqrt{\left|v-Q_{0}\right|}} \mathrm{e}\left(\lambda, v ; Q_{0}\right) d \lambda \\
= & 2 \int_{-\infty}^{-E_{1} \sqrt{\left|v-Q_{0}\right|}} \lambda^{-2} \exp \left(2\left(v-Q_{0}\right) / \lambda\right)\left(1+O\left(\lambda^{-2}\right)+O\left(\left|v-Q_{0}\right| \lambda^{-3}\right)\right) d \lambda \\
= & 2 \int_{-1 /\left(E_{1} \sqrt{\left|v-Q_{0}\right|}\right)}^{0} \exp \left(2 \lambda\left(v-Q_{0}\right)\right)\left(1+O\left(\lambda^{2}\right)+O\left(\left|v-Q_{0}\right| \lambda^{3}\right)\right) d \lambda \\
= & \frac{1}{v-Q_{0}}+O\left(\left|v-Q_{0}\right|^{-3}\right) .
\end{aligned}
$$

Summing the contributions of the three ranges up gives the desired result and also shows the asymptotic behavior required in (ii).

(ii) The difference $f$ of two discrete Cauchy's kernels with respect to $Q_{0}$ of asymptotics $o\left(\left|v-Q_{0}\right|^{-1 / 2}\right)$ is discrete holomorphic and fulfills the conditions of Theorem 2.31. Hence, $f$ is biconstant, so $f \equiv 0$.

(iii) Let $b_{-}, w_{-}, b_{+}, w_{+}$denote the vertices of the parallelogram $Q \in V(\diamond)$ in counterclockwise order, starting with a black vertex. Let us introduce $a:=w_{+}-b_{-}$ and $b:=w_{-}-b_{-}$. Using the expansion $1 /(1+x)=1-x+O\left(x^{2}\right)$ as $x \rightarrow 0$ and the asymptotics of the first part, we get that $K_{Q_{0}}\left(b_{ \pm}\right)$and $K_{Q_{0}}\left(w_{ \pm}\right)$equal 


$$
\begin{aligned}
& \frac{1}{Q-Q_{0}} \mp \frac{a+b}{2\left(Q-Q_{0}\right)^{2}}+\frac{\tau\left(b_{-}, Q_{0}\right)}{J\left(Q, Q_{0}\right)} \mp \tau\left(b_{-}, Q_{0}\right) \frac{a^{-1}+b^{-1}}{2 J\left(Q, Q_{0}\right)^{2}}+O\left(\left|Q-Q_{0}\right|^{-3}\right), \\
& \frac{1}{Q-Q_{0}} \mp \frac{a-b}{2\left(Q-Q_{0}\right)^{2}}+\frac{\tau\left(w_{-}, Q_{0}\right)}{J\left(Q, Q_{0}\right)} \mp \tau\left(w_{-}, Q_{0}\right) \frac{a^{-1}-b^{-1}}{2 J\left(Q, Q_{0}\right)^{2}}+O\left(\left|Q-Q_{0}\right|^{-3}\right),
\end{aligned}
$$

respectively. By definition, $\tau\left(b_{-}, Q_{0}\right)=-\tau\left(w_{-}, Q_{0}\right)$. Therefore,

$$
\begin{aligned}
\partial_{\Lambda} K_{Q_{0}}(Q)= & -\lambda Q \frac{\frac{a+b}{\left(Q-Q_{0}\right)^{2}}-\tau\left(b_{-}, Q_{0}\right) \frac{a^{-1}+b^{-1}}{J\left(Q, Q_{0}\right)^{2}}}{a+b} \\
& +\bar{\lambda} Q \frac{\frac{a-b}{\left(Q-Q_{0}\right)^{2}}-\tau\left(b_{-}, Q_{0}\right) \frac{a^{-1}-b^{-1}}{J\left(Q, Q_{0}\right)^{2}}}{a-b}+O\left(\left|Q-Q_{0}\right|^{-3}\right) \\
= & -\frac{1}{\left(Q-Q_{0}\right)^{2}}-\frac{\tau\left(b_{-}, Q_{0}\right)}{a b J\left(Q, Q_{0}\right)^{2}}+O\left(\left|Q-Q_{0}\right|^{-3}\right) \\
= & -\frac{1}{\left(Q-Q_{0}\right)^{2}}-\frac{\tau\left(Q, Q_{0}\right)}{J\left(Q, Q_{0}\right)^{2}}+O\left(\left|Q-Q_{0}\right|^{-3}\right) .
\end{aligned}
$$

(iv) Let $f$ be the difference of two discrete Cauchy's kernels with respect to $Q_{0}$ whose discrete derivatives have asymptotics $o\left(\left|Q-v_{0}\right|^{-1 / 2}\right)$. Then, $f$ is discrete holomorphic and

$$
\frac{f\left(b_{+}\right)-f\left(b_{-}\right)}{b_{+}-b_{-}}=\frac{f\left(w_{+}\right)-f\left(w_{-}\right)}{w_{+}-w_{-}}=\partial_{\Lambda} f(Q)=o\left(\left|Q-v_{0}\right|^{-1 / 2}\right) .
$$

Since angles and edge lengths are bounded, the conditions of Theorem 2.31 are fulfilled. Hence, $f$ is biconstant.

Remark Note that Kenyon [16] and Chelkak and Smirnov [6] proved in the rhombic setting the stronger result that there is a unique discrete Cauchy's kernel on $V(\Lambda)$ with respect to $Q_{0}$ with asymptotics $o(1)$ as $|v| \rightarrow \infty$.

\subsection{Integer Lattice}

Let us consider a planar parallelogram-graph $\Lambda$ such that each vertex has degree four. With the embedding of $\diamond$ described in Sect. 3.1, this happens if and only if $\diamond$ is a planar quad-graph or, equivalently, if $\Lambda$ has the combinatorics of the integer lattice $\mathbb{Z}^{2}$. The vertices of $\diamond$ lie at the midpoints of edges of $\Gamma$ or $\Gamma^{*}$. Since any vertex of $\Gamma$ or $\Gamma^{*}$ is enclosed by a quadrilateral of $\Gamma^{*}$ or $\Gamma$, respectively, the faces of $\diamond$ are parallelograms by Varignon's theorem. Thus, $\diamond$ becomes a planar parallelogramgraph as well.

Of particular interest is the case that the two notions of discrete holomorphicity on $\diamond$, the one coming from $\diamond$ being the dual of $\Lambda$ and the other coming from the quadgraph $\diamond$ itself, coincide. It is not hard to show that this happens only for the integer 
lattice of a skew coordinate system, onto which we restrict ourselves in the following. For more details, see [13]. If $e_{1}, e_{2}$ denote two spanning vectors, then $\diamond$ is a parallel shift of $\Lambda$ by $e_{1} / 2+e_{2} / 2$. Furthermore, the discrete derivatives on $\nabla$ seen as the dual of $\Lambda$ coincide with the discrete derivatives on $\diamond$ seen as a parallelogram-graph.

Since corresponding notions coincide and $\diamond$ and $\Lambda$ are congruent, we can skip all subscripts $\Lambda$ and $\diamond$ in the definitions of discrete derivatives. Moreover, the discrete Laplacian $\triangle$ is now defined for functions on $V(\Lambda)$ and functions on $V(\diamond)$ in the same way. Due to Corollary $2.20,4 \partial \bar{\partial}=\Delta=4 \bar{\partial} \partial$ is now true on both graphs. It follows that all discrete derivatives $\partial^{n} f$ of a discrete holomorphic function $f$ are discrete holomorphic themselves. Conversely, a discrete primitive exists for any discrete holomorphic function on a simply-connected domain by Proposition 2.8.

Our main interests lie in giving discrete Cauchy's integral formulae for higher order derivatives of a discrete holomorphic function and determining the asymptotics of higher order discrete derivatives of the discrete Cauchy's kernel given in Sect. 3.4. Note that due to the uniqueness statements in Theorems 3.8 and 3.10, both formulae yield the same discrete Cauchy's kernel.

Without loss of generality, we restrict our attention to functions on $V(\Lambda)$. For the ease of notation, we introduce the discrete distance $D(\cdot, \cdot)$ on $V(\Lambda) \cup V(\diamond)$ that is induced by the $|\cdot|_{\infty}$-distance on the integer lattice spanned by $e_{1} / 2, e_{2} / 2$.

Theorem 3.11 Let $\Lambda$ be the integer lattice spanned by the pair $e_{1}, e_{2}$ of linearly independent complex vectors. Let $v_{0} \in V(\Lambda), Q_{0}:=v_{0}+e_{1} / 2+e_{2} / 2 \in V(\diamond)$, let $f$ be a discrete holomorphic function on $V(\Lambda)$, and let $K_{v_{0}}$ and $K_{Q_{0}}$ be discrete Cauchy's kernels with respect to $v_{0}$ and $Q_{0}$, respectively. Let $n$ be a nonnegative integer and define $x_{0}:=v_{0}$ if $n$ is even and $x_{0}:=Q_{0}$ if $n$ is odd. Similarly, let $x \in V(\Lambda)$ if $n$ is even and $x \in V(\diamond)$ if $n$ is odd.

(i) For any counterclockwise oriented discrete contour $C_{x_{0}}$ in the medial graph $X$ enclosing all points $x^{\prime} \in V(\Lambda) \cup V(\diamond)$ with $D\left(x^{\prime}, x_{0}\right) \leqslant n / 2$,

$$
\partial^{n} f\left(x_{0}\right)=\frac{(-1)^{n}}{2 \pi i} \oint_{C_{x_{0}}} f \partial^{n} K_{x_{0}} d z .
$$

(ii) If $K_{Q_{0}}$ is the discrete Cauchy's kernel given in Proposition 3.9, then

$$
\begin{aligned}
& \frac{(-1)^{n}}{n !} \partial^{n} K_{Q_{0}}(x)=\frac{1}{\left(x-Q_{0}\right)^{n+1}}+\frac{\tau^{\prime}\left(x, Q_{0}\right)}{\left(J\left(x, Q_{0}\right) e_{1} e_{2}\right)^{n+1}}+O\left(\left|x-Q_{0}\right|^{-n-3}\right) \\
& \text { as }|x| \rightarrow \infty, \text { where } \tau^{\prime}\left(x, Q_{0}\right)=1 \text { if } x \text { and } Q_{0} \text { or }\left(x+e_{1} / 2+e_{2} / 2\right) \text { and } Q_{0} \text { can } \\
& \text { be connected by a path on } V(\diamond) \text { of even length and } \tau^{\prime}\left(x, Q_{0}\right)=-1 \text { otherwise. } \\
& \text { Constants in the } O \text {-notation depend on the spanning vectors } e_{0}, e_{1} \text { only. }
\end{aligned}
$$


Remark By Lemma 3.6(ii) and (iii), we have for $|x| \rightarrow \infty$ :

$$
\frac{\tau^{\prime}\left(x, Q_{0}\right)}{\left(J\left(x, Q_{0}\right) e_{1} e_{2}\right)^{n+1}}=\Omega\left(\left(x-Q_{0}\right)^{-(n+1)}\right) .
$$

Proof (i) Let $D$ be the discrete domain in $F(X)$ bounded by $C_{x_{0}}$. By the assumptions on $C_{x_{0}}$, the discrete one-form $\bar{\partial} \partial^{n-1} K_{x_{0}} d \bar{z}$ vanishes on $C_{x_{0}}$. Thus,

$$
\oint_{C_{x_{0}}} f \partial^{n} K_{x_{0}} d z=\oint_{C_{x_{0}}} f d\left(\partial^{n-1} K_{x_{0}}\right)=\iint_{D} d\left(f d\left(\partial^{n-1} K_{x_{0}}\right)\right)=\iint_{D} d f \wedge d\left(\partial^{n-1} K_{x_{0}}\right)
$$

by discrete Stokes' Theorem 2.9 in the second equation and Theorem 2.16 and Proposition 2.10 stating that $d$ is a derivation for the discrete wedge product and $d d f=0$ in the third equation. Now, $f$ is discrete holomorphic, meaning that $\bar{\partial} f \equiv 0$, so $d f \wedge d\left(\partial^{n-1} K_{x_{0}}\right)=\partial f \bar{\partial} \partial^{n-1} K_{x_{0}} \Omega_{\diamond}$. But since the discrete derivatives commute according to Corollary 2.11, $\bar{\partial} \partial^{n-1} K_{x_{0}}=\partial^{n-1} \bar{\partial} K_{x_{0}}$ vanishes outside $C_{x_{0}}$, so by repeated use of Proposition 2.7 stating that $\partial$ is the formal adjoint of $-\bar{\partial}$,

$$
\oint_{C_{x_{0}}} f \partial^{n} K_{x_{0}} d z=-2 i\left\langle\partial f, \bar{\partial}^{n-1} \partial \bar{K}_{x_{0}}\right\rangle=2 i(-1)^{n}\left\langle\partial^{n} f, \partial \bar{K}_{x_{0}}\right\rangle=2 \pi i(-1)^{n} \partial^{n} f\left(x_{0}\right) .
$$

(ii) Let us define the discrete exponential e $\left(\lambda, Q ; Q_{0}\right)$ for $Q \in V(\diamond)$ in the same way as a rational function in the complex variable $\lambda$ as we defined $\mathrm{e}\left(\lambda, v ; v_{0}\right)$. By inductive use of the formula for the discrete derivative of exp in Proposition 3.2 and $\exp \left(\lambda, \cdot ; Q_{0}\right)=\mathrm{e}\left(2 / \lambda, \cdot ; Q_{0}\right)$, we get for the discrete exponential e $\left(\lambda, \cdot ; Q_{0}\right)$ that is defined on $V(\Lambda)$ :

$$
\left(\partial^{n} \mathrm{e}\left(\lambda, ; Q_{0}\right)\right)(x)=\frac{(2 \lambda)^{n}}{\left(\left(\lambda-e_{1}\right)\left(\lambda-e_{2}\right)\left(\lambda+e_{1}\right)\left(\lambda+e_{2}\right)\right)^{\lceil n / 2\rceil}} \mathrm{e}\left(\lambda, x ; Q_{0}\right) .
$$

To compute $\partial^{n} K_{Q_{0}}(x)$, we need the values of $K_{Q_{0}}$ only at vertices $v \in V(\Lambda)$ with $D(v, x) \leqslant n / 2$. For these points,

$$
K_{Q_{0}}(v)=2 \int_{-\left(v-Q_{0}\right) \infty}^{0} \mathrm{e}\left(\lambda, v ; Q_{0}\right) d \lambda
$$

by the formula in Proposition 3.9. If $D\left(x, Q_{0}\right)$ is sufficiently large, then we can choose $e \in\left\{e_{1}, e_{2},-e_{1},-e_{2}\right\}$ such that $D(k e, x)>n / 2$ for all positive integers $k$. So if $D(v, x) \leqslant n / 2$ and $D\left(x, Q_{0}\right)$ is sufficiently large, then $\mathrm{e}\left(\cdot, v ; Q_{0}\right)$ does not have the pole $e$. Since $\pm e_{1}, \pm e_{2}$ are the only possible poles, it follows that there are no poles in the convex hull of all the rays $\left(-\left(v-Q_{0}\right) \infty, 0\right]$ for the vertices $v$ with $D(v, x) \leqslant n / 2$. By residue theorem, we can use the same path of integration for all relevant values of $K_{Q_{0}}$ and get 


$$
\partial^{n} K_{Q_{0}}(x)=2 \int_{-\left(x-Q_{0}\right) \infty}^{0}\left(\partial^{n} \mathrm{e}\left(\lambda, \cdot ; Q_{0}\right)\right)(x) d \lambda .
$$

Now, we follow the proof of Theorem 3.10(i). Again, the claim is invariant under rotation of the complex plane, so we may assume $\left(x-Q_{0}\right)>0$.

Let $E_{1}:=\max \left\{\left|e_{1}\right|,\left|e_{2}\right|\right\}$ and $E_{0}:=\min \left\{\left|e_{1}\right|,\left|e_{2}\right|\right\}$. For $\left|x-Q_{0}\right| \geqslant 1$ large enough, we split the integration into the three parts along $\left(-\infty,-E_{1} \sqrt{\left|x-Q_{0}\right|}\right]$, $\left[-E_{1} \sqrt{\left|x-Q_{0}\right|},-E_{1} / \sqrt{\left|x-Q_{0}\right|}\right]$, and $\left[-E_{1} / \sqrt{\left|x-Q_{0}\right|}, 0\right]$.

By Lemma 3.6(iv), the contribution of $\lambda \in\left[-E_{1} \sqrt{\left|x-Q_{0}\right|},-E_{1} / \sqrt{\left|x-Q_{0}\right|}\right]$ decays faster to zero than any power of $x-Q_{0}$ as $|x| \rightarrow \infty$. By Lemma 3.6(i) and (ii), we know that $D\left(x, Q_{0}\right)=\Omega\left(x-Q_{0}\right)$ and $J\left(x, Q_{0}\right)=\Omega\left(x-Q_{0}\right)$. Furthermore, the choice of $\left(x-Q_{0}\right)>0$ implies that $\operatorname{Re}\left(J\left(x, Q_{0}\right)\right)=\Omega\left(x-Q_{0}\right)$ stays positive as $|x| \rightarrow \infty$.

Let $\lambda \in\left[-E_{1} / \sqrt{\left|x-Q_{0}\right|}, 0\right]$. Then, $\lambda \rightarrow 0$ as $|x| \rightarrow \infty$, and repeated use of the second and third equation in Lemma 3.5(i) gives that $\left(\partial^{n} \mathrm{e}\left(\lambda, \cdot ; Q_{0}\right)\right)(x)$ equals

$$
\frac{\tau^{\prime}\left(x, Q_{0}\right)}{\left(e_{1} e_{2}\right)^{n+1}}(2 \lambda)^{n} \exp \left(2 \lambda J\left(x, Q_{0}\right)\right)\left(1+O\left(\lambda^{2}\right)+O\left(\left|x-Q_{0}\right| \lambda^{3}\right)\right) .
$$

With a similar argument as in the proof of Theorem 3.10(i), the integral near the origin behaves for $|x| \rightarrow \infty$ as

$$
\frac{(-1)^{n} n ! \tau^{\prime}\left(x, Q_{0}\right)}{\left(e_{1} e_{2} J\left(x, Q_{0}\right)\right)^{n+1}}+O\left(\left|x-Q_{0}\right|^{-n-3}\right) .
$$

Now, let $\lambda \in\left(-\infty,-E_{1} \sqrt{\left|x-Q_{0}\right|}\right]$. For $|x| \rightarrow \infty, \lambda \rightarrow-\infty$, and repeated use of the second and third equation in Lemma 3.5(ii) implies that $\left(\partial^{n} \mathrm{e}\left(\lambda, \cdot ; Q_{0}\right)\right)(x)$ equals

$$
(2 \lambda)^{n} \lambda^{-2 n-2} \exp \left(\frac{2\left(x-Q_{0}\right)}{\lambda}\right)\left(1+O\left(\lambda^{-2}\right)+O\left(\left|x-Q_{0}\right| \lambda^{-3}\right)\right) .
$$

Using the result of above, we get for the integral near minus infinity for $|x| \rightarrow \infty$ :

$$
\begin{aligned}
& \int_{-\infty}^{-E_{1} \sqrt{\left|x-Q_{0}\right|}}\left(\partial^{n} \mathrm{e}\left(\lambda, \cdot ; Q_{0}\right)\right)(x) d \lambda \\
= & 2 \int_{-\infty}^{-E_{1} \sqrt{\left|x-Q_{0}\right|}}\left(\frac{2}{\lambda}\right)^{n} \lambda^{-2} \exp \left(\frac{2\left(x-Q_{0}\right)}{\lambda}\right)\left(1+O\left(\lambda^{-2}\right)+O\left(\left|x-Q_{0}\right| \lambda^{-3}\right)\right) d \lambda
\end{aligned}
$$




$$
\begin{aligned}
& =2 \int_{-1 /\left(E_{1} \sqrt{\left|x-Q_{0}\right|}\right)}^{0}(2 \lambda)^{n} \exp \left(2 \lambda\left(x-Q_{0}\right)\right)\left(1+O\left(\lambda^{2}\right)+O\left(\left|x-Q_{0}\right| \lambda^{3}\right)\right) d \lambda \\
& =\frac{(-1)^{n} n !}{\left(x-Q_{0}\right)^{n+1}}+O\left(\left|x-Q_{0}\right|^{-n-3}\right) .
\end{aligned}
$$

Summing up the contributions of the three ranges gives

$$
\frac{(-1)^{n}}{n !} \partial^{n} K_{Q_{0}}(x)=\frac{1}{\left(x-Q_{0}\right)^{n+1}}+\frac{\tau^{\prime}\left(x, Q_{0}\right)}{\left(J\left(x, Q_{0}\right) e_{1} e_{2}\right)^{n+1}}+O\left(\left|x-Q_{0}\right|^{-n-3}\right) .
$$

Acknowledgments The authors thank Richard Kenyon, Christian Mercat, and Mikhail Skopenkov for fruitful discussions and helpful suggestions. In addition, we are grateful to Mikhail Skopenkov for his detailed review of previous versions and his numerous and valuable recommendations. The first author was partially supported by the DFG Collaborative Research Center TRR 109, "Discretization in Geometry and Dynamics". The research of the second author was supported by the Deutsche Telekom Stiftung. Some parts of this paper were written at the IHÉS in Buressur-Yvette, the INIMS in Cambridge, and the ESI in Vienna. The second author thanks the EPDI program for the opportunity to stay at these institutes. The stay at the Isaac Newton Institute for Mathematical Sciences was funded through an Engineering and Physical Sciences Research Council Visiting Fellowship, Grant EP/K032208/1.

\section{Appendix: Planar Parallelogram-Graphs}

The aim of this appendix is to discuss some combinatorial and geometric properties of parallelogram-graphs that were used in Sect. 3. The following notion of a strip is standard, see for example the book [3].

Definition A strip in a planar quad-graph $\Lambda$ is a path on its dual $\diamond$ such that two successive faces share an edge and the strip leaves a face in the opposite edge where it enters it. Moreover, strips are assumed to have maximal length, i.e., there are no strips containing it apart from itself.

Note that a strip is uniquely determined by the edges it passes through, meaning the edges two successive faces share.

Definition For a strip $S$ of a parallelogram-graph $\Lambda$, there exists a complex vector $a_{S}$ such that any (nonoriented) edge through which $S$ passes is $\pm a_{S}$. We call $a_{S}$ a common parallel.

$a_{S}$ is unique up to sign; the choice of the sign induces an orientation on all edges. The parallel edges of the strip can be rescaled to length $\left|a_{S}\right|=1$, without changing the combinatorics. Hence, rhombic planar quad-graphs and planar parallelogram-graphs are combinatorially equivalent. Rhombic planar quad-graphs are characterized by the following proposition of Kenyon and Schlenker [17]: 
Proposition 4.1 A planar quad-graph $\Lambda$ admits a combinatorially equivalent embedding in $\mathbb{C}$ with all rhombic faces if and only if the following two conditions are satisfied:

- No strip crosses itself or is periodic.

- Two distinct strips cross each other at most once.

Remark Let us prove the simpler claim that planar parallelogram-graphs fulfill these two conditions as was already noted by Kenyon in [16]. The underlying reason is that any strip $S$ is monotone with respect to the direction $i a_{S}$ : The coordinates of the endpoints of the edges parallel to $a_{S}$ are strictly increasing or strictly decreasing if they are projected to $i a_{S}$. Whether the projections are decreasing or increasing depends on the direction in which the faces of $S$ are passed through. Without loss of generality, we assume that the faces of $S$ are passed through in such a way that the projections of the corresponding coordinates are strictly increasing. For $Q \in S$, let $S^{Q}$ denote the semi-infinite part of $S$ starting in the quadrilateral $Q$ that passes through the faces of $S$ in the same order.

As a consequence, no strip crosses itself or is periodic. Furthermore, $S$ divides the complex plane into two unbounded regions, to one is $a_{S}$ pointing and to the other $-a_{S}$. When a distinct strip $S^{\prime}$ crosses $S$, it enters a different region determined by $S$, say it goes to the one to which $a_{S}$ is pointing. Due to monotonicity, the angle between $i a_{S^{\prime}}$ and $a_{S}$ is less than $\pi / 2$. It follows that $S^{\prime}$ cannot cross $S$ another time, since it would then go to the region $-a_{S}$ is pointing to, contradicting that the angle between $i a_{S^{\prime}}$ and $-a_{S}$ is greater and not less than $\pi / 2$.

In order to give explicit formulae for the discrete Green's function and the discrete Cauchy's kernels in Sects. 3.3 and 3.4, we chose a particular directed path connecting two vertices (or a face and a vertex) by edges of the parallelogram-graph $\Lambda$. This path was monotone in one direction. The existence of such a path follows from the following lemma, generalizing a result of [2] to general parallelogram-graphs. The proof bases on the same ideas.

Lemma 4.2 Let $\Lambda$ be a parallelogram-graph and let $v_{0} \in V(\Lambda)$ be fixed. For a directed edge e of $\Lambda$ starting in $v_{0}$, consider the subgraph $U_{e} \subset \Lambda$ that consists of all vertices and edges of directed paths on $\Lambda$ starting in $v_{0}$ whose oriented edges have arguments that can be chosen to lie in $[\arg (e), \arg (e)+\pi)$.

Then, the union of all $U_{e}$, e a directed edge starting in $v_{0}$, covers the whole quadgraph. If there is a constant $\alpha_{0}>0$ such that $\alpha \geqslant \alpha_{0}$ for all interior angles $\alpha$ of faces of $\Lambda$, then the same statement holds true if $[\arg (e), \arg (e)+\pi)$ is replaced by $\left[\arg (e), \arg (e)+\pi-\alpha_{0}\right]$.

Proof Let us rescale the edges such that all of them have length one. By this, we change neither the combinatorics of $\Lambda$ nor the size of interior angles.

For a directed edge $e$ starting in $v_{0}$, let $U_{e}^{-}$and $U_{e}^{+}$denote the (directed) paths on $\Lambda$ starting in $v_{0}$, obtained by choosing the directed edge with the least or largest 
$\operatorname{argument}$ in $[\arg (e), \arg (e)+\pi)\left(\operatorname{or}\left[\arg (e), \arg (e)+\pi-\alpha_{0}\right]\right)$ at a vertex, respectively. We first show that all vertices in between $U_{e}^{-}$and $U_{e}^{+}$are contained in $V\left(U_{e}\right)$. Then, it follows that $U_{e}$ is the conical sector with boundary $U_{e}^{-}$and $U_{e}^{+}$.

Suppose the contrary, i.e., suppose that there is a vertex $v$ between $U_{e}^{-}$and $U_{e}^{+}$to which $v_{0}$ cannot be connected by a directed path of edges whose arguments lie all in the interval $[\arg (e), \arg (e)+\pi)\left(\operatorname{or}\left[\arg (e), \arg (e)+\pi-\alpha_{0}\right]\right)$. Let the combinatorial distance between $v_{0}$ and $v$ be minimal among all such vertices.

In the case that interior angles of rhombi are bounded by $\alpha_{0}$ from below, they are bounded from above by $\pi-\alpha_{0}$. Hence, there is a vertex $v^{\prime}$ adjacent to $v$ such that the argument of the directed edge $v^{\prime} v$ lies in $\left[\arg (e), \arg (e)+\pi-\alpha_{0}\right]$. Even if interior angles are not uniformly bounded, $v^{\prime}$ can be chosen in such a way that the argument of $v^{\prime} v$ lies in $[\arg (e), \arg (e)+\pi)$. By construction, $v^{\prime}$ is not in $V\left(U_{e}\right)$, but still between $U_{e}^{-}$and $U_{e}^{+}$. Let us look at the strip $S$ passing through $v^{\prime} v$. Suppose that the common parallel $a_{S}$ points from $v^{\prime}$ to $v$.

If $S$ intersects $U_{e}^{-}$or $U_{e}^{+}$, then an edge parallel to $a_{S}$ is contained in $U_{e}^{-}$or $U_{e}^{+}$, respectively. By construction, $v_{0}$ and $v^{\prime}$ then lie on the same side of the strip $S$.

If $S$ does neither intersect $U_{e}^{-}$nor $U_{e}^{+}$, then it is completely contained in the left half space determined by the oriented line $v_{0}+t e, t \in \mathbb{R}$, as $U_{e}^{-}$and $U_{e}^{+}$are. Suppose $S$ intersects the ray $v_{0}+t a_{S}, t \geqslant 0$. Again, it follows that $v_{0}$ and $v^{\prime}$ lie on the same side of $S$.

It remains the case that $S$ neither intersects $U_{e}^{-}, U_{e}^{+}$, nor the ray $v_{0}+t a_{S}, t \geqslant 0$. Consider the quadrilateral area $R$ in between the parallels $v_{0}+t a_{S}, v^{\prime}+t a_{S}$ and $v_{0}+t e, v^{\prime}+t e, t \in \mathbb{R}$. By assumption, the semi-infinite part of $S$ that starts with the edge $v^{\prime} v$ and then goes into $R$ does not intersect an edge of $R$ incident to $v_{0}$, and by monotonicity, it does not intersect $v^{\prime}+t a_{S}$ again. Now, $\Lambda$ is locally finite, such that only finitely many quadrilaterals of $S$ are inside $P$. Thus, $S$ leaves $P$ on the edge $v^{\prime}+t e, t \in \mathbb{R}$, and it follows that $S$ separates $v_{0}$ and $v$.

So in any case, $S$ separates $v_{0}$ from $v$. Any shortest path $P$ connecting both points has to pass through $S$. Let $w$ be the first point of $P$ that lies on the same side of $S$ as $v$ does. Any strip passing through an edge on the shortest path connecting $w$ and $v$ on $S$ has to intersect $P$ as well. It follows that replacing all edges of $P$ on the same side of $S$ as $v$ by the path from $w$ to $v$ does not change its length. But then, $v^{\prime}$ is combinatorially nearer to $v_{0}$ than $v$, contradiction.

Finally, we can cyclically order the directed edges starting in $v_{0}$ according to their slopes. Then, the sectors $U_{e}$ are interlaced, i.e., $U_{e}$ contains both $U_{e_{-}}^{+}$and $U_{e_{+}}^{-}$, where $e_{-}, e, e_{+}$are consecutive according to the cyclic order. As a consequence, the union of all these $U_{e}$ covers the whole $\Lambda$.

To perform our computations in Sects. 3.3 and 3.4, we needed not only that the interior angles were bounded, but also that the side lengths were bounded. We can relax the latter condition to boundedness of the ratio of side lengths.

Proposition 4.3 Let $\Lambda$ be a parallelogram-graph and assume that there are constants $\alpha_{0}, q_{0}>0$ such that $\alpha \geqslant \alpha_{0}$ and $e / e^{\prime} \geqslant q_{0}$ for all interior angles $\alpha$ and two side lengths $e, e^{\prime}$ of any parallelogram of $\Lambda$. Then, $E_{1} \geqslant e \geqslant E_{0}$ for all edge lengths 
$e$, where $E_{0}:=e_{0} q_{0}^{N}$ and $E_{1}:=e_{1} q_{0}^{-N}$ with $N:=\left\lceil\left\lfloor 2 \pi / \alpha_{0}\right\rfloor / 2\right\rceil$ and $e_{1} \geqslant e_{0}$ being the side lengths of an arbitrary parallelogram of $\Lambda$.

Proof Let $Q^{\prime} \in V(\diamond)$ be fixed with edge lengths $e_{1} \geqslant e_{0}$ and let $Q \in V(\diamond)$ be another parallelogram with center $x$. In the following, we construct a sequence of $n$ strips such that any two consecutive strips are crossing each other, the first one contains $Q^{\prime}$, the last one contains $Q$, and $n \leqslant N$. Then, it follows that the side lengths of $Q$ are bounded by $E_{0}$ and $E_{1}$.

Let $S_{0}$ be a strip containing $Q^{\prime}$. If $Q \in S_{0}$, we are done. Otherwise, we choose the common parallel $a_{S_{0}}$ in such a way that $x$ lies in the region $-a_{S_{0}}$ is pointing to. Let $\beta_{0}:=\arg \left(a_{S_{0}}\right)$. Since $S_{0}$ is monotone in the direction $i a_{S_{0}}$ and interior angles are uniformly bounded, the ray $x+t a_{S_{0}}, t>0$, intersects $S$ in exactly one line segment. Let $y_{0}$ be the first intersection point and $Q_{0}$ a quadrilateral of $S$ containing $y_{0}$.

Because $\Lambda$ is locally finite, the line segment connecting $x$ and $y_{0}$ intersects only finitely many parallelograms. Through any such parallelogram at most two strips are passing. Thus, only a finite number of strips intersect this line segment. Therefore, we can choose a strip $S_{1}$ intersecting $S_{0}^{Q_{0}}$ in a parallelogram $Q_{0,1}$ such that $S_{1}$ does not contain $Q$ and does not intersect the line segment connecting $x$ and $y_{0}$. Moreover, we require that $Q^{\prime} \notin S_{0}^{Q_{0,1}}$. Now, choose the common parallel $a_{S_{1}}$ of $S_{1}$ in such a way that there is an argument $\beta_{1}$ of $a_{S_{1}}$ that satisfies $\pi+\beta_{0}>\beta_{1}>\beta_{0}$. By construction, $x$ lies in the region $-a_{S_{1}}$ is pointing to. Note that $S_{1}^{Q_{0,1}}$ cannot cross $S_{0}$ a second time.

Suppose we have already constructed the strip $S_{k}$ with common parallel $a_{S_{k}}$ and argument $\beta_{k}, k>0$, and $x$ lies in the region $-a_{S_{k}}$ is pointing to. $S_{k}$ shall not intersect the line segments connecting $x$ and $y_{0}$ or connecting $x$ and $y_{k-1}$. Moreover, assume that the semi-infinite part $S_{k}^{Q_{k-1, k}}$ starting in the intersection $Q_{k-1, k}$ of $S_{k}$ with $S_{k-1}$ does not cross $S_{0}$.

Let $y_{k}$ be the first intersection of the ray $x+t a_{S_{k}}, t>0$, with a quadrilateral $Q_{k}$ of the strip $S_{k}$. By the same arguments as above, there exists a strip $S_{k+1}$ intersecting $S_{k}^{Q_{k-1, k}} \cap S_{k}^{Q_{k}}$ that does not contain $Q$ and does not intersect the line segments connecting $x$ and $y_{0}$ or $x$ and $y_{k}$. Choose its common parallel $a_{S_{k+1}}$ in such a way that it has an argument $\beta_{k+1}$ that satisfies $\pi+\beta_{k}>\beta_{k+1}>\beta_{k}$. By construction, $x$ lies in the region $-a_{S_{k+1}}$ is pointing to. If the semi-infinite part $S_{k+1}^{Q_{k, k+1}}$ starting in the intersection $Q_{k, k+1}$ with $S_{k}$ does not cross $S_{0}$, then we continue this procedure. For a schematic picture of the proof, see Fig. 7.

After at most $l:=\left\lceil 2 \pi / \alpha_{0}\right\rceil$ steps, we end up with a strip $S_{l}$ such that $S_{l}^{Q_{l-1, l}}$ intersects $S_{0}$. Indeed, let us suppose the contrary, that is, let us suppose that all $S_{2}^{Q_{1,2}}, \ldots, S_{l}^{Q_{l-1, l}}$ do not cross $S_{0}$.

By assumption, $\beta_{k}+\pi-\alpha_{0} \geqslant \beta_{k+1} \geqslant \beta_{k}+\alpha_{0}$. It follows that the first $j$ such that $\beta_{j}$ is greater or equal than $\beta_{0}+2 \pi$ satisfies $j \leqslant\left\lceil 2 \pi / \alpha_{0}\right\rceil$. In addition, we have $\beta_{j}<\beta_{0}+3 \pi-\alpha_{0}$.

By construction, $S_{j}$ does not intersect the line segment connecting $x$ and $y_{j-1}$. Moreover, $S_{j}^{Q_{j-1, j}}$ cannot cross $S_{j-1}$ a second time. It follows that $S_{j}^{Q_{j-1, j}}$ cannot intersect the ray $x+t a_{S_{j-1}}, t>0$. 


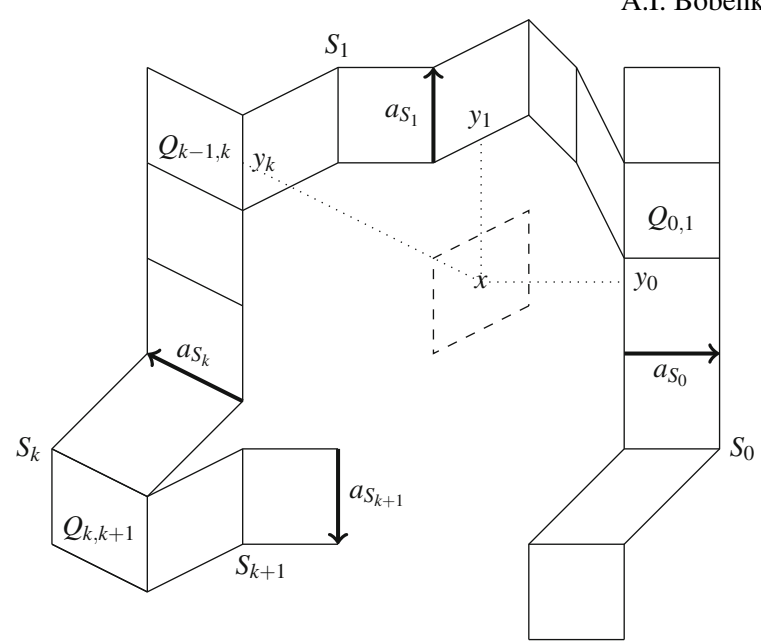

Fig. 7 Schematic picture of the proof of Proposition 4.3

Also, $S_{j}^{Q_{j-1, j}}$ does neither cross $S_{0}$ nor does it intersect the line segment connecting $x$ and $y_{0}$, so it does not intersect the ray $x+t a_{S_{0}}, t>0$. Thus, $S_{j}^{Q_{j-1, j}}$ is contained in the cone with tip $x$ spanned by $a_{S_{j-1}}$ and $a_{S_{0}}$ (with angle less than $\pi$ ). This contradicts the monotonicity of $S_{j}^{Q_{j-1, j}}$ into the direction $i a_{S_{j}}$, because the ray $x+t a_{S_{j}}, t>0$, is not contained in the interior of the cone above.

In summary, we found a cycle of $m$ strips $S_{0}, S_{1}, \ldots, S_{m-1}$ surrounding $x$, where $m \leqslant\left\lfloor 2 \pi / \alpha_{0}\right\rfloor+1$. Actually, $m \leqslant\left\lfloor 2 \pi / \alpha_{0}\right\rfloor$, because the $a_{S_{k}}$ are cyclically ordered. Since only finitely many strips intersect the strip $S_{0}$ in between $Q^{\prime}$ and $Q_{0,1}$, we can assume that $Q^{\prime}$ is contained in $S_{0}^{Q_{m-1,0}}$.

These $m$ strips determine a bounded region $x$ is contained in. If $Q^{\prime} \neq Q_{m-1,0}$, then we look at the semi-infinite part of the strip $\tilde{S}_{0}$ different from $S_{0}$ that passes through $Q^{\prime}$ and goes into the interior of the bounded region above. It has to intersect one of the strips $S_{1}, \ldots, S_{m-1}$, say $S_{k}$. Then, $S_{0}, \ldots, S_{k}, \tilde{S}_{0}$ or $\tilde{S}_{0}, S_{k}, \ldots, S_{m-1}, S_{0}$ determine a bounded region $x$ is contained in ( $Q$ may be an element of $\tilde{S}_{0}$ ). Clearly, they are at most $\left\lfloor 2 \pi / \alpha_{0}\right\rfloor$ such strips, and $Q^{\prime}$ lies on an intersection.

If $Q \notin \tilde{S}_{0}$, then a strip $S_{Q}$ containing $Q$ has to cross two different strips of the cycle due to local finiteness. In the same way as above, we can find a cycle of at most $m^{\prime} \leqslant\left\lfloor 2 \pi / \alpha_{0}\right\rfloor$ strips $S_{0}^{\prime}, S_{1}^{\prime}, \ldots, S_{m^{\prime}-1}^{\prime}$ such that $Q$ lies on one of the strips, say $S_{k}^{\prime}$, and the intersection of $S_{0}^{\prime}$ and $S_{m^{\prime}-1}^{\prime}$ is $Q^{\prime}$. If $k \leqslant\left\lceil m^{\prime} / 2\right\rceil$, then we choose the sequence of strips $S_{0}^{\prime}, S_{1}^{\prime}, \ldots, S_{k}^{\prime}$; otherwise, we take $S_{m^{\prime}-1}^{\prime}, S_{m^{\prime}-2}^{\prime}, \ldots, S_{k}^{\prime}$. Any two consecutive strips are crossing each other, $Q^{\prime}$ is on the first strip, $Q$ on the last one, and there are at most $\left\lceil\left\lfloor 2 \pi / \alpha_{0}\right\rfloor / 2\right\rceil$ of them.

Remark In general, the bound $\left\lceil\left\lfloor 2 \pi / \alpha_{0}\right\rfloor / 2\right\rceil$ in the proof is optimal. Indeed, consider $n$ rays emanating from 0 such that the angle between any two neighboring rays is $2 \pi / n$. In each of the $n$ segments, choose the quad-graph combinatorially equivalent to the positive octant of the integer lattice that is spanned by two consecutive rays. 
For example, if $n=4$, we obtain $\mathbb{Z}^{2}$. Then, any strip passes through exactly two adjacent segments, and $\lceil n / 2\rceil$ is the optimal bound.

Open Access This chapter is distributed under the terms of the Creative Commons AttributionNoncommercial 2.5 License (http://creativecommons.org/licenses/by-nc/2.5/) which permits any noncommercial use, distribution, and reproduction in any medium, provided the original author(s) and source are credited.

The images or other third party material in this chapter are included in the work's Creative Commons license, unless indicated otherwise in the credit line; if such material is not included in the work's Creative Commons license and the respective action is not permitted by statutory regulation, users will need to obtain permission from the license holder to duplicate, adapt or reproduce the material.

\section{References}

1. Bobenko, A., Günther, F.: Discrete Riemann surfaces based on quadrilateral cellular decompositions (2015). Preprint arXiv: 1511.00652

2. Bobenko, A., Mercat, C., Suris, Y.: Linear and nonlinear theories of discrete analytic functions. Integrable structure and isomonodromic Green's function. J. Reine Angew. Math. 583, 117-161 (2005)

3. Bobenko, A., Suris, Y.: Discrete differential geometry: integrable structure. In: Graduate Studies in Mathematics, vol. 98. AMS, Providence (2008)

4. Brooks, R., Smith, C., Stone, A., Tutte, W.: The dissection of rectangles into squares. Duke Math. J. 7(1), 312-340 (1940)

5. Bücking, U.: Approximation of conformal mappings by circle patterns. Geom. Dedicata 137, 163-197 (2008)

6. Chelkak, D., Smirnov, S.: Discrete complex analysis on isoradial graphs. Adv. Math. 228, 1590-1630 (2011)

7. Chelkak, D., Smirnov, S.: Universality in the 2D Ising model and conformal invariance of fermionic observables. Invent. Math. 189(3), 515-580 (2012)

8. Courant, R., Friedrichs, K., Lewy, H.: Über diepartiellen Differenzengleichungen der mathematischen Physik. Math. Ann. 100, 32-74 (1928)

9. Duffin, R.: Basic properties of discrete analytic functions. Duke Math. J. 23(2), 335-363 (1956)

10. Duffin, R.: Potential theory on a rhombic lattice. J. Comb. Th. 5, 258-272 (1968)

11. Dynnikov, I., Novikov, S.: Geometry of the triangle equation on two-manifolds. Moscow Math. J. 3(2), 419-482 (2003)

12. Ferrand, J.: Fonctions préharmoniques et fonctions préholomorphes. Bull. Sci. Math. Ser. 2(68), 152-180 (1944)

13. Günther, F.: Discrete Riemann surfaces and integrable systems. Ph.D. thesis, Technische Universität Berlin (2014). http://opus4.kobv.de/opus4-tuberlin/files/5659/guenther_felix.pdf

14. Isaacs, R.: A finite difference function theory. Univ. Nac. Tucumán. Rev. A 2, 177-201 (1941)

15. Kenyon, R.: Conformal invariance of domino tiling. Ann. Prob. 28(2), 759-795 (2002)

16. Kenyon, R.: The Laplacian and Dirac operators on critical planar graphs. Invent. Math. 150, 409-439 (2002)

17. Kenyon, R., Schlenker, J.M.: Rhombic embeddings of planar quad-graphs. Trans. Amer. Math. Soc. 357(9), 3443-3458 (2005)

18. Lelong-Ferrand, J.: Représentation conforme et transformations à intégrale de Dirichlet bornée. Gauthier-Villars, Paris (1955) 
19. Mercat, C.: Discrete Riemann surfaces and the Ising model. Commun. Math. Phys. 218(1), 177-216 (2001)

20. Mercat, C.: Discrete Riemann surfaces. In: Handbook of Teichmüller theory. Vol. I, IRMA Lect. Math. Theor. Phys., vol. 11, pp. 541-575. Eur. Math. Soc., Zurich (2007)

21. Mercat, C.: Discrete complex structure on surfel surfaces. Proceedings of the 14th IAPR International Conference on Discrete Geometry for Computer Imagery. DGCI'08, pp. 153-164. Springer-Verlag, Berlin, Heidelberg (2008)

22. Rodin, B., Sullivan, D.: The convergence of circle packings to the Riemann mapping. J. Diff. Geom. 26(2), 349-360 (1987)

23. Skopenkov, M.: The boundary value problem for discrete analytic functions. Adv. Math. 240, 61-87 (2013)

24. Smirnov, S.: Discrete complex analysis and probability. In: Proceedings of the International Congress of Mathematicians 2010 (ICM 2010), vol. I: Plenary Lectures and Ceremonies, vols. II-IV: Invited Lectures, pp. 595-621. Hindustan Book Agency, New Delhi, India (2000)

25. Smirnov, S.: Critical percolation in the plane: conformal invariance, Cardy's formula, scaling limits. C. R. Math. Acad. Sci. Paris Sér. I 333(3), 239-244 (2001)

26. Smirnov, S.: Conformal invariance in random cluster models. I. Holomorphic fermions in the Ising model. Ann. Math. 172(2), 1435-1467 (2010)

27. Whitney, H.: On products in a complex. Ann. Math. 39(2), 397-432 (1938) 